\title{
DERIVED CATEGORIES AND GROTHENDIECK DUALITY
}

\author{
AMNON NEEMAN
}

\begin{abstract}
We study dualizing complexes. The unusual feature is that we do not assume them to have bounded injective resolutions; we prove that the theory works just fine with no boundedness hypothesis. In the process we prove a number of new results about Grothendieck duality; one of the more striking is that, under relatively mild hypotheses on $f: X \longrightarrow Y$, the functor $f^{!}: \mathbf{D}(\mathrm{Qcoh} / Y) \longrightarrow$ $\mathbf{D}(\mathrm{Qcoh} / X)$ takes pseudocoherent complexes to pseudocoherent complexes. The biggest innovation in our approach is that we systematically employ products in the category $\mathbf{D}(\mathrm{Qcoh} / X)$; the older treatments never ventured beyond coproducts.

In an appendix we include a proof that, if $\mathcal{T}$ is a stable homotopy category in the sense of [19] in which the compact objects coincide with the strongly dualizable objects, then the compact objects can also be characterized as those objects such that tensoring with them respects products.
\end{abstract}

\section{Contents}

$0 . \quad$ Introduction

1. Historical overview

2. Background on $\mathbf{R}$ Hom complexes 12

3. Dualizing complexes 24

4. When $\mathbf{R} f_{*}$ respects compacts 34

5. Where we can prove Conjecture 4.16 47

6. Dualizing complexes and $f^{!}$

7. Several recent results 54

Appendix A. A fact concerning strongly dualizable objects $\quad 54$

References $\quad 56$

Key words and phrases. dualizing complex, Grothendieck duality.

The research was partly supported by the Australian Research Council. 


\section{InTRODUCTION}

The bulk of this survey will give a modern account of dualizing complexes. Let $X$ be a noetherian, separated scheme. Let $\mathbf{D}^{b}(\mathrm{Coh} / X)$ be the bounded derived category of coherent sheaves on $X$. We define a dualizing complex to be an object $\mathcal{J} \in \mathbf{D}^{b}(\mathrm{Coh} / X)$ so that the natural functor

$$
\mathbf{R} \mathcal{H o m}(-, \mathcal{J}): \mathbf{D}^{b}(\mathrm{Coh} / X)^{\text {op }} \longrightarrow \mathbf{D}^{b}(\mathrm{Coh} / X)
$$

is an equivalence of categories. Note that we make no assumption that $\mathcal{J}$ have a bounded injective resolution; this is supposed to be a contemporary treatment of the subject, and by now we should know how to handle unbounded complexes. The thrust is that the theory works just fine. Let me now sketch the main new results which can be found in the article.

Facts 0.1. We prove, with no simplifying hypotheses on $\mathcal{J}$ :

(i) A dualizing complex $\mathcal{J}$, if it exists, is unique up to the obvious modifications. That is, if $\mathcal{J}$ and $\mathcal{J}^{\prime}$ are two dualizing complexes, then there exists a complex $\mathcal{L} \in \mathbf{D}^{b}(\mathrm{Coh} / X)$ with $\mathcal{J}^{\prime} \cong \mathcal{L}^{\mathbf{L}} \otimes \mathcal{J}$. Furthermore, on any connected component of $X$ the complex $\mathcal{L}$ is just a suspension of a line bundle. See Lemma 3.9 for the proof.

(ii) An object $\mathcal{J} \in \mathbf{D}^{b}(\mathrm{Coh} / X)$ is a dualizing complex if and only if it satisfies the two conditions

(a) The natural map $\mathcal{O}_{X} \longrightarrow \mathbf{R} \mathcal{H}$ om $(\mathcal{J}, \mathcal{J})$ is an isomorphism.

(b) For every object $\varepsilon \in \mathbf{D}^{b}(\mathrm{Coh} / X)$ we have

$$
\mathbf{R} \mathcal{H o m}(\mathcal{E}, \mathcal{J}) \in \mathbf{D}^{b}(\mathrm{Coh} / X) .
$$

For a proof see Proposition 3.6.

The traditional reason why people cared about dualizing complexes was the way they behave in the relative situation, where two schemes are involved; we will discuss this a little more fully in $\S 6$. For now we confine ourselves to stating our relevant new results. Suppose therefore that we are given a morphism $f: X \longrightarrow Y$, where $X$ and $Y$ are noetherian, separated schemes. Let $\mathbf{D}(\mathrm{Q} \operatorname{coh} / X)$ and $\mathbf{D}(\mathrm{Qcoh} / Y)$ be the unbounded derived categories of quasicoherent sheaves on $X$ and $Y$ respectively. There is a pushforward functor $\mathbf{R} f_{*}: \mathbf{D}(\mathrm{Q} \operatorname{coh} / X) \longrightarrow \mathbf{D}(\mathrm{Q} \operatorname{coh} / Y)$, and it has a left adjoint $\mathbf{L} f^{*}$ and a right adjoint $f^{!}$. The next results are

Facts 0.2. Let $\mathcal{J}$ be a dualizing complex in $\mathbf{D}^{b}(\mathrm{Coh} / Y) \subset \mathbf{D}(\mathrm{Q} \operatorname{coh} / Y)$. Then the following is true:

(i) If $f$ is an open immersion, then $\mathbf{L} f^{*} \mathcal{J}$ is a dualizing complex in $\mathbf{D}^{b}(\mathrm{Coh} / X) \subset \mathbf{D}(\mathrm{Q} \operatorname{coh} / X)$. The proof is in Theorem 3.12.

(ii) Suppose $f: X \longrightarrow Y$ satisfies the following two hypotheses: (a) $\mathbf{R} f_{*}$ takes $\mathbf{D}^{b}(\mathrm{Coh} / X)$ to $\mathbf{D}^{b}(\mathrm{Coh} / Y)$. 
(b) $f^{!}$takes $\mathbf{D}^{b}(\mathrm{Coh} / Y)$ to $\mathbf{D}_{\mathrm{Coh}}^{+}(\mathrm{Q} \operatorname{coh} / X)$.

Then $f^{!} \mathcal{J}$ is a dualizing complex on $X$. The proof may be found in Theorem 3.14.

Fact 0.2 (ii) is a little unsatisfactory, particularly because of the hypothesis (b) which involves the mysterious functor $f^{!}$. The next fact helps.

Facts 0.3. Hypotheses (a) and (b) of Fact 0.2(ii) hold in either of the following two situations:

(i) The morphism $f: X \longrightarrow Y$ is finite. Hypothesis (a) holds obviously, and the proof that hypothesis (b) is satisfied may be found in Lemma 3.18.

(ii) The functor $\mathbf{R} f_{*}: \mathbf{D}(\mathrm{Qcoh} / X) \longrightarrow \mathbf{D}(\mathrm{Qcoh} / Y)$ takes perfect complexes to perfect complexes, and $X$ satisfies the technical condition ${ }^{*}$ ) of Conjecture 4.16. Hypothesis (a) holds by [46, Corollary 4.3.2], and for hypothesis (b) see Corollary 0.7.

As always if $X \stackrel{f}{\longrightarrow} Y \stackrel{g}{\longrightarrow} Z$ are two morphism, and if both $f^{!}$and $g^{!}$take dualizing complexes to dualizing complexes, then so does the composite. We can freely combine (i) and (ii) to produce morphisms $h$ for which $h^{\text {! }}$ respects dualizing complexes.

Remark 0.4. I do not, at the present time, fully understand the technical condition $(*)$ in Fact $0.3(\mathrm{ii})$. Conjecture 4.16 articulates the hope that $\left(^{*}\right.$ ) holds for all $X$. We rephrase this slightly: assuming that the technical Conjecture 4.16 holds for every $X$, then $f^{!}$takes dualizing complexes to dualizing complexes as long as $\mathbf{R} f_{*}: \mathbf{D}(\mathrm{Qcoh} / X) \longrightarrow \mathbf{D}(\mathrm{Qcoh} / Y)$ takes perfect complexes to perfect complexes.

Given that at present we do not have Conjecture 4.16 in this generality, in addition to the preservation of perfect complexes we currently need to assume that Conjecture 4.16 holds for $X$. In the remainder of the introduction I will discuss the status of Conjecture 4.16; there are many $X \mathrm{~s}$ for which it is known.

Until now all our schemes were assumed noetherian. Dualizing complexes are traditionally about producing equivalences $\mathbf{D}^{b}(\mathrm{Coh} / X)^{\mathrm{op}} \cong \mathbf{D}^{b}(\mathrm{Coh} / X)$, and the category $\mathbf{D}^{b}(\mathrm{Coh} / X)$ does not obviously make sense unless $X$ is noetherian, or at least coherent. But it turns out that Conjecture 4.16, as well as the technical condition $\left(^{*}\right)$ in Fact $0.3($ ii), make sense much more generally. For the next result we only assume that our schemes are quasicompact and separated. In the article we will prove the following assertion

Facts 0.5. Let $f: X \longrightarrow Y$ be a morphism of quasicompact, separated schemes. Assume that Conjecture 4.16 holds for $X$, and that $\mathbf{R} f_{*}$ takes 
perfect complexes to perfect complexes. Then the complex $f^{!} \mathcal{O}_{Y}$ is pseudocoherent; this means that, for any open affine subset $U \subset X$, the restriction of $f^{!} O_{Y}$ to $U=\operatorname{Spec}(R)$ is quasi-isomorphic to the sheafification of a bounded above chain complex of finitely generated, projective $R$-modules.

The proof is in Lemma 4.21; see also Remark 4.8.

Remark 0.6. By [51, Theorem 5.4] we know that $f^{!} \mathcal{S} \cong f^{!} \mathcal{O}_{Y} \mathbf{L} \otimes \mathbf{L} f^{*} \mathcal{S}$. If $\mathcal{S}$ is pseudocoherent then so is $\mathbf{L} f^{*} \mathcal{S}$ (obviously), and the formula, coupled with the pseudocoherence of $f^{!} \mathcal{O}_{Y}$ of Fact 0.5 , informs us that $f^{!} \mathcal{S}$ is also pseudocoherent.

Assume now that $X$ and $Y$ are noetherian and $\mathcal{S}$ belongs to $\mathbf{D}^{b}(\mathrm{Coh} / Y)$. Then $f^{!} \mathcal{S}$ is pseudocoherent, which for noetherian schemes simply means it belongs to $\mathbf{D}^{-}(\mathrm{Coh} / X)$. The fact that its cohomology is bounded below is easy (see Remark 3.13) and we conclude the following:

Corollary 0.7. Assume $f: X \longrightarrow Y$ is a morphism of noetherian, separated schemes. Suppose further that $\mathbf{R} f_{*}$ takes perfect complexes to perfect complexes, and that Conjecture 4.16 holds for $X$. Then $f^{!}$takes $\mathbf{D}^{b}(\mathrm{Coh} / Y)$ to $\mathbf{D}^{b}(\mathrm{Coh} / X)$.

In Remark 0.6 we sketched the argument leading from the more general Fact 0.5 to Corollary 0.7, valid in the case of noetherian schemes. The reader should note that Fact 0.3 (ii) is immediate from Corollary 0.7. Corollary 0.7 proves more than we need; in Fact 0.3(ii) we only asserted that $f^{!}$takes $\mathbf{D}^{b}(\mathrm{Coh} / Y)$ to $\mathbf{D}_{\mathrm{Coh}}^{+}(\mathrm{Q} \operatorname{coh} / X)$.

Facts 0.8. It remains to review what we know about Conjecture 4.16. The current state of knowledge is that $X$ satisfies the conjecture if

(i) $X$ is noetherian, finite dimensional, and smooth over a finite dimensional, noetherian regular ring $R$. See Theorem 5.13 for the proof.

(ii) $X$ is a locally closed subscheme of $Y$, and Conjecture 4.16 is true for $Y$.

(iii) There is an affine morphism $X \longrightarrow Y$, and Conjecture 4.16 is true for $Y$. Facts (ii) and (iii) both follow from Remark 5.12.

We leave it to the imagination of the reader to combine these to produce many $X \mathrm{~s}$ for which the conjecture is true.

This finishes the main new results we will present; it remains to give a brief sketch of the structure of the article. We begin with a fairly extensive historical review of Grothendieck duality. Then we cover the basic properties of $\mathbf{R} \mathcal{H o m}(-,-)$; nothing here is especially new, but our approach is to define $\mathbf{R} \mathcal{H} \operatorname{Hom}(\mathcal{E},-)$ as the right adjoint of the functor $-\mathbf{L}_{\otimes} \mathcal{E}$, by appealing to Brown representability. It is therefore a little interesting to see that the theory can be developed quickly and painlessly; this approach is 
relatively new, it first appeared in Murfet's thesis [48, Appendix C]. Then the rest of the article is devoted to proving the claims we have made in the Introduction. The one comment that might be helpful is that our approach hinges on systematically using products in the categories $\mathbf{D}(\mathrm{Q} c \mathrm{ch} / X)$. These categories are compactly generated, and therefore have products; see [52, Proposition 8.4.6]. Any right adjoint will preserve these products, and the functors $\mathbf{R} \mathcal{H} \operatorname{om}(\mathcal{E},-), \mathbf{R} f_{*}$ and $f^{!}$are all right adjoints. The idea is to exploit this.

If $X$ is arbitrary then products in the category $\mathbf{D}(\mathrm{Qcoh} / X)$ are disgusting; we only really understand them when $X$ is affine. As we will see, this is often enough. One can frequently reduce oneself to the affine case.

Acknowledgements. The author would like to thank Avramov, Iyengar, Joyal, Krause, Lipman, Murfet, Street and Van den Bergh for their valuable suggestions towards improving an earlier draft. Lipman sent me a long list of helpful comments, and Avramov and Iyengar even went as far as providing a simplified, streamlined proof for Lemma 2.10; the current manuscript contains their proof.

\section{Historical overview}

Triangulated categories came into being in the early 1960s; they arose independently, and more or less simultaneously, in the work of Puppe [55] and of Verdier $[67]^{1}$. Puppe's interest in the subject came from homotopy theory; we will say more, much later in this historical account, about the role homotopy theory played in the early development of the subject. Our survey of the early history will follow in the footsteps of Verdier; we will explain the problem that inspired him, and the progress that followed. Verdier came to the subject with a specific goal in mind: he wanted to develop the necessary homological machinery to state and prove Grothendieck duality.

Let us permit ourselves a digression, jumping ahead many years in time: since the early days much has happened, and derived categories, or more generally triangulated categories, have successfully invaded branches of mathematics as remote as mathematical physics $[9,18,30,31]$ and even $\mathbb{C}^{*}$-algebras [47]; the other articles in this volume are testament to the astounding success of the field. My aims are more modest; in this survey we will keep our focus on Grothendieck duality. What we will illustrate is the way in which the gradual advances in our understanding of the foundations of derived categories, over many years, have translated into improvements in the results on Grothendieck duality.

\footnotetext{
${ }^{1}$ The reader should note that Verdier's thesis was only published posthumously, many years after it was written; the publication date of [67] is misleading.
} 
Let us return to the dawn of the field; in the beginning there was the Serre duality theorem [60]. In its original form the theorem states the following.

Theorem 1.1. Serre [60]. Let $X$ be a connected, $n$-dimensional compact complex manifold, and let $\mathcal{V}$ be a holomorphic vector bundle on $X$. Then the dual of the vector space $H^{i}(X, \mathcal{V})$ is isomorphic to $H^{n-i}\left(X, \mathcal{H} \operatorname{Hom}\left(\mathcal{V}, \Omega^{n}\right)\right)$.

Remark 1.2. Perhaps we should explain the notation. The line bundle $\Omega^{n}$ is the bundle of holomorphic $n$-forms on $X$, and $\mathcal{H o m}\left(\mathcal{V}, \Omega^{n}\right)$ is the vector bundle of whose sections, on an open set $U \subset X$, are the holomorphic maps of vector bundles $\left.\left.\mathcal{V}\right|_{U} \longrightarrow \Omega^{n}\right|_{U}$. There is an obvious pairing

$$
H^{i}(X, \mathcal{V}) \otimes H^{n-i}\left(X, \mathcal{H} \operatorname{Com}\left(\mathcal{V}, \Omega^{n}\right)\right) \stackrel{\varphi}{\longrightarrow} H^{n}\left(\Omega^{n}\right),
$$

and Serre's theorem asserts two things:

(i) The vector space $H^{n}\left(\Omega^{n}\right)$ is one dimensional.

(ii) The pairing $\varphi$ is perfect; it gives a natural identification of $H^{n-i}\left(X, \mathcal{H} \operatorname{Hom}\left(\mathcal{V}, \Omega^{n}\right)\right)$ with the dual of $H^{i}(X, \mathcal{V})$.

The question that interested people at the time was whether there is a relative version. Suppose we are given a holomorphic map of complex manifolds $f: X \longrightarrow Y$. Is there a reasonable general theorem which, in the special case where $Y=\{*\}$ is the one-point space, comes down to Theorem 1.1? The answer turns out to be Yes, at least in the algebro-geometric framework. In keeping with tradition I will now switch from the complex analytic setting to the world of algebraic geometry; I do this because complex analysis has technical difficulties which I do not want to address.

Let $X$ be a smooth, $n$-dimensional projective variety over a field $k$, and let $\mathcal{V}$ be an algebraic vector bundle on $X$. Serre duality, in its algebraic variety context, gives a natural isomorphism

$$
\operatorname{Hom}\left(H^{i}(X, \mathcal{V}), k\right) \longrightarrow H^{n-i}\left(X, \mathcal{H} \operatorname{com}\left(\mathcal{V}, \Omega^{n}\right)\right) \text {. }
$$

To make life a little more exciting let us choose $W$, a finite dimensional vector space over the field $k$. Clearly we also have a natural isomorphism

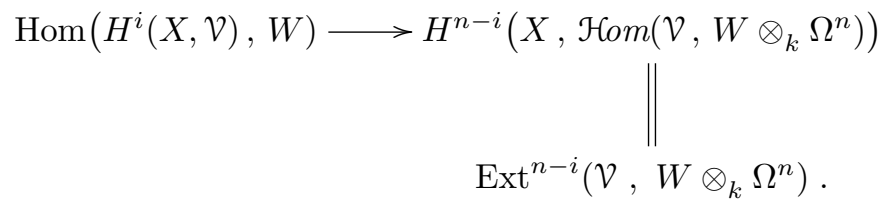

Now the vector space $W$ can be thought of as a vector bundle over the one-point space $Y=\{*\}$, and, if you squint hard enough, the isomorphism above begins to look like an adjunction. We have a functor $\mathbf{R} f_{*}$, which takes a vector bundle $\mathcal{V}$ on $X$ to a string of vector bundles on $Y$, namely the $H^{i}(X, \mathcal{V})$. And we have the functor $f^{!}$taking the vector bundle $W$ on $Y$ 
to $W \otimes_{k} \Omega^{n}$. If we are willing to treat the Ext's as Hom's and to disregard the confusing indices $i$ and $(n-i)$, it looks like we have an isomorphism

$$
\operatorname{Hom}\left(\mathbf{R} f_{*} \mathcal{V}, W\right) \longrightarrow \operatorname{Hom}\left(\mathcal{V}, f^{!} W\right) .
$$

The problem was to make this intuition precise. Before all else, it would be necessary to say exactly what $\nu_{\mathbf{S}}$ are allowed as inputs for the functor $\mathbf{R} f_{*}$, and what $W$ s as inputs for the functor $f^{!}$. We need to come up with two categories. One, which we will provisionally label $\mathbb{D}(X)$, should consist of some sort of sheaves over $X$. The other, for which our tentative name will be $\mathbb{D}(Y)$, will be made up of something like sheaves over $Y$. And we want functors $\mathbf{R} f_{*}: \mathbb{D}(X) \longrightarrow \mathbb{D}(Y)$ and $f^{!}: \mathbb{D}(Y) \longrightarrow \mathbb{D}(X)$, with $f^{!}$right adjoint to $\mathbf{R} f_{*}$. And finally we want all of this to generalize Serre duality. The objects of the category $\mathbb{D}(X)$ should include the vector bundles on $X$, and $\mathbf{R} f_{*} \mathcal{V}$ should be something which encapsulates the information in the string of vector spaces $\left\{H^{i}(X, \mathcal{V}), 0 \leq i \leq n\right\}$.

In his 1958 talk at the Edinburgh ICM (see [13]) Grothendieck announced that he had a solution, but also that the homological algebra language necessary to state it did not yet exist. Verdier's thesis project was to develop the framework. Derived categories were born in that thesis. The idea that worked, as we now know, was to let $\mathbb{D}(X)$ and $\mathbb{D}(Y)$ be derived categories; the objects are chain complexes of sheaves on $X$ and $Y$ respectively, and the morphisms are the chain maps, with the homology isomorphisms formally inverted.

Remark 1.3. Let us remind the reader. If $f: X \longrightarrow Y$ is a morphism of schemes, then there is always an induced map $f_{*}$, taking sheaves on $X$ to sheaves on $Y$. It extends to complexes of sheaves: given a complex $\mathcal{V}$ of sheaves on $X$, we can form a complex $f_{*} \mathcal{V}$ of sheaves on $Y$. The complex $\mathbf{R} f_{*} \mathcal{V}$ is obtained by first replacing $\mathcal{V}$ by an injective resolution, and then applying $f_{*}$. It is, on the face of it, quite strange that this functor should have a right adjoint. The reason this is a little bizarre is that the functor $f_{*}$ is left exact, but decidedly not right exact. We would expect right adjoints to exist only for right exact functors, and therefore $f_{*}$ most certainly cannot have a right adjoint. Only at the level of the derived categories, after replacing $f_{*}$ by $\mathbf{R} f_{*}$, do we have a hope of finding an $f^{!}$. The passage, from the abelian category of chain complexes to its derived category, is very brutal; it destroys exactness. We will return to this point in Remarks 1.6 and 1.7. For now we note only that the brutality is vital, without it Grothendieck duality wouldn't stand a chance.

Remark 1.4. Right from the start people disliked derived categories. Derived categories were quite unlike the more familiar objects of homological 
algebra, their behaviour was poorly understood, and people felt uncomfortable using them. The consensus was that there had to be a better, more natural substitute for them. People felt that derived categories couldn't possibly be the real answer, they couldn't be the right framework for all these theorems. To this day the attitude persists; there are many people who are still trying, today, to find a more natural foundation for this branch of homological algebra. A huge amount of exciting work has come out in the last few years. The potential replacements for derived categories include DG-categories, $\mathcal{A}_{\infty}$-categories, stable model categories, Segal spaces, quasicategories and triangulated derivators.

Because people never liked the formalism, derived categories were slow to catch on. Now, almost fifty years later, we can point to the impressive theorems that have been proved using them, and to the extent to which they have come to permeate far-flung branches of mathematics. As I have already said, the other chapters in this book contain a compelling case for their success. At this point we can safely say that, for a theory that everyone badmouthed from the very outset, derived categories have come a long way.

In Remark 1.4 we mentioned that people have always had a distaste for derived categories. It might help if we explain one of the features that no one likes. We begin with

Lemma 1.5. Let $\mathcal{T}$ be a triangulated category. Then any epimorphism $f: X \longrightarrow Y$ in $\mathcal{T}$ is split.

Proof. Complete $f$ to a distinguished triangle

$$
X \stackrel{f}{\longrightarrow} Y \stackrel{g}{\longrightarrow} Z \stackrel{h}{\longrightarrow} \Sigma X .
$$

We know that the composite $g f: X \longrightarrow Z$ vanishes, and that $f$ is an epimorphism. Hence $g$ must vanish. From [52, Corollary 1.2.7] it follows that the triangle is isomorphic to

$$
Y \oplus \Sigma^{-1} Z \stackrel{(1,0)}{\longrightarrow} Y \stackrel{0}{\longrightarrow} Z \longrightarrow \Sigma Y \oplus Z,
$$

and hence $f: X \longrightarrow Y$ must be a split epimorphism.

Remark 1.6. Suppose we have a morphism $f: X \longrightarrow Y$, and suppose it has a cokernel $g: Y \longrightarrow Q$. We observe first that the map $g$ must be an epimorphism. Lemma 1.5 guarantees that it is a split epimorphism: $Y$ is isomorphic to $I \oplus Q$, where I is the (split) kernel of $g$. The map $f: X \longrightarrow Y$ therefore identifies as a composite

$$
X \stackrel{\alpha}{\longrightarrow} I \longrightarrow I \oplus Q .
$$

The fact that the projection $I \oplus Q \longrightarrow Q$ is the cokernel of $f$ guarantees that $\alpha: X \longrightarrow I$ must be an epimorphism. Using Lemma 1.5 again, we conclude 
that $\alpha$ must split. We have shown that, if a morphism $f: X \longrightarrow Y$ has a cokernel, then it must factor as

$$
X \stackrel{\alpha}{\longrightarrow} I \stackrel{\beta}{\longrightarrow} Y \text {, }
$$

with $\alpha$ a split epimorphism and $\beta$ a split monomorphism. In homological algebra people had spent half a century taking kernels and cokernels of maps, and suddenly they were faced with the world of triangulated categories, where such constructions are worthless. No wonder they were unhappy.

Remark 1.7. Let us look at a slightly fancier version of this, where we consider other possible colimits in triangulated categories. It is easy to see, by example, that there is nothing to prevent the existence of coproducts; there are many examples of triangulated categories with coproducts, for instance the unbounded derived category of modules over a ring $R$. We say that such categories satisfy [TR5].

Now let $\mathcal{T}$ be a [TR5] triangulated category. If $F: S \longrightarrow \mathcal{T}$ is a functor from a small category $S$ to $\mathcal{T}$, we can wonder whether it has a colimit. Suppose such a colimit exists. Now observe that the natural map

$$
\coprod_{s \in S} F(s) \longrightarrow \underset{\operatorname{colim}}{\longrightarrow} F
$$

must be an epimorphism. Lemma 1.5 guarantees that this epimorphism has to split. We conclude that, aside from coproducts, there can be no interesting colimits in $\mathcal{T}$. The special case of cokernels is only a baby example of a much more serious problem.

In Remark 1.4 we mentioned that derived categories were not exactly a welcome introduction to homological algebra, while in Lemma 1.5 and Remarks 1.6 and 1.7 we explained one of the features that people have found undesirable. The Almighty Lord, in His infinite grace and wisdom, has not seen fit to bestow upon me the gift of clairvoyant, prophetic powers; it is not for me to predict if someone really will succeed in finding a superior replacement, whether it will be one of the contenders currently being promoted, and if so which one. Only time will tell.

Back to Grothendieck duality. We have recounted how derived categories were born, to establish a context in which Grothendieck's duality theorem could be stated and proved. The proof appeared in Hartshorne [15]; the book dates back to 1966 . Why am I wasting the reader's time with a 40-yearold theorem? Surely everything about it must be completely understood by now. The volume in front of you is meant to deal with current and future mathematics, not with ancient history.

Well, Hartshorne's book may have appeared in 1966, but back then derived categories were barely out of diapers, their coordination was less than 
perfect, and they were still throwing temper tantrums whenever anyone tried to take them into new territory. They were very new, poorly understood, and the technical apparatus created for dealing with them was crude and clumsy. We have come a long way since then, and probably have an equally long road ahead of us. There is little doubt that, forty years from now, people looking back at the work we do today will find it laughably awkward and roundabout. We cannot escape the fact that derived categories are work-in-progress, and many tools are presumably yet to be conceived. This being the case we are still learning new facts about Grothendieck duality. The main object of this article is to give an updated account of dualizing complexes.

This is true, most of the article will be modern, but it would be wrong not to mention the valuable contributions that people have made over the decades. In the early years there was a great deal of interest in the subject; the theorem was new and hot and mathematicians, like the rest of humanity, throng around the newest hot topic on the block. Work from that period includes (of course) Hartshorne [15], but also Deligne [7, 8], Grothendieck [14], Illusie [23, 24, 25, 26], Ramis, Ruget and Verdier [56] and Verdier [64, 65, 66].

After the early explosion of interest the subject had a long hiatus. There was a period of almost two decades when only a small group of committed specialists kept the flame burning: results form this period include El Zein [11], Grivel [12], Hopkins [16], Hopkins-Lipman [17], HüblSastry [22], Hübl-Kunz [20, 21], Kunz [36, 37], Kunz-Waldi [39], Kiehl [34], Lipman [42, 43], Van den Bergh [63] and Yekutieli [68, 69]. But it was only in the late 1980's that the foundations of derived categories began undergoing a major change, with a concommitant improvement in our understanding of Grothendieck duality.

In the early days, people working on derived categories preferred to consider their bounded incarnations. It was customary to work with $\mathbf{D}^{+}(\mathcal{A})$, $\mathbf{D}^{-}(\mathcal{A})$ or $\mathbf{D}^{b}(\mathcal{A})$; we remind the reader that these are, respectively, the bounded below, bounded above or bounded derived categories. Depending on the problem at hand people were quite willing to switch around among the three bounded versions, but the one derived category that was only rarely touched was the unbounded derived category $\mathbf{D}(\mathcal{A})$. There was good reason for this: except in very special cases it was not understood how to form projective or injective resolutions of unbounded complexes, and hence there was no clear machinery for producing derived functors. Perhaps the first article to alter matters was Spaltenstein [61]. This 1988 paper is very methodical; it carefully outines how to go about this, explaining in some detail the various resolutions one can form, and discussing the situations 
to which they are best suited. The terminology we use today is still the one Spaltenstein introduced, for example when we speak about $K$-injective resolutions.

The second shift came a little later, with $[4,50,51]$. It began as a pure accident; I happened to be visiting Bielefeld, as a fellow of the Humboldt Stiftung, in the academic year 1989-90. My office was just down the corridor from that of Marcel Bökstedt, who is a wonderful mathematician, a clear thinker and an excellent expositor, a man with the gift of explaining the key idea in what would otherwise be a foggy, abstruse formalism. I enjoyed talking to him and learned a great deal from the interaction, and in particular I learned from Bökstedt what little homotopy theory I know. As he was explaining this to me I was struck by the parallel with the mathematics I knew, the mathematics of algebraic geometry. Our joint article [4] arose out of these conversations; it amounts to my translation, to the familiar context of algebraic geometry, of the homotopy theory that Bökstedt was teaching me.

Maybe we should elaborate a little. In Remarks 1.6 and 1.7 we noted that there are few interesting colimits in derived categories. The homotopy theorists have never let this bother them; they freely lift problems from triangulated categories to model categories. In the model categories colimit constructions work just fine, and occasionally one can show that the output of these constructions is independent of the lifting; one has produced something well-defined in the triangulated category. It is often not difficult to reformulate the constructions in a way that dispenses with the lifting to models. Anyway, all we really want to stress is that the homotopy theorists walked fearlessly through terrain which the derived categorists carefully skirted. I learned from Bökstedt that there were paths in this unfamiliar territory, and the reader may find this in [4]. When we worked on [4], Bökstedt and I were unaware of Spaltenstein's earlier [61], and we thought we were being very clever when we applied the techniques of homotopy theory to show how one can easily handle unbounded complexes. Unfortunately for us Spaltenstein had achieved the same, a few years earlier, and without using any ideas from homotopy theory.

In the next few years I came to better appreciate the power of the methods, and found a couple of new applications $[50,51]^{2}$, results which did not tread in the footsteps of Spaltenstein. The techniques then caught on

\footnotetext{
${ }^{2}$ Once again the publication dates are misleading; the later paper [50] was published before [4], which took longer to be accepted. With [50] I happened to be lucky; Thomason was the referee, liked the result and accepted it immediately. Also, [50] and [51] were written at about the same time, but published four year apart; [51] took forever to be accepted.
} 
with other authors, and not only in the context of Grothendieck duality; for example one of the early converts was Bernhard Keller, whose article [32] proved very influential. Somewhat later, Hovey, Palmieri and Strickland's monograph [19] helped by making the foundations more accessible to the non-homotopy-theorists. This survey confines itself to Grothendieck duality; in that field, the papers which most successfully employed homotopytheoretic methods were Alonso, Jeremías and Lipman [1, 2] Alonso, Jeremías and Souto [3], Jørgensen [28] and Lipman [41]. It should be mentioned that our understanding of what might be possible, using homotopy theoretic methods and other techniques, is not yet complete; there is ongoing work, for example $[6,38,44,45,46,49,58,59,70,71]$.

This ends our glimpse of history, more precisely the brief overview of the early history of Grothendieck duality. Next we begin talking mathematics; first we will review for the reader the basics of dualizing complexes.

\section{BACKGROUND ON RHFm COMPLEXES}

Let us quickly review some basic facts about the internal Hom in the derived category $\mathbf{D}(\mathrm{Q} \operatorname{coh} / X)$. The results are not far from standard, but perhaps the perspective is slightly unusual: we construct $\mathbf{R} \mathcal{H}$ om using Brown representability, and then obtain all the properties we need by reducing to the affine case. Throughout this section $X$ will be a quasicompact, separated scheme, and much of the time we will also assume it noetherian. The category $\mathbf{D}(\mathrm{Qcoh} / X)$ will be the unbounded derived category of quasicoherent sheaves on $X$.

Remark 2.1. Instead of $\mathbf{D}(\mathrm{Qcoh} / X)$ we could look at $\mathbf{D}_{\mathrm{Qcoh}}(X)$, the derived category whose objects are complexes of sheaves of $\mathcal{O}_{X}$-modules with quasicoherent cohomology. When $X$ is separated and quasicompact, the obvious functor $\mathbf{D}(\mathrm{Q} \operatorname{coh} / X) \longrightarrow \mathbf{D}_{\mathrm{Qcoh}}(X)$ is an equivalence of categories, but for more general $X$ the categories can be different. For schemes that are only quasiseparated it turns out that the category $\mathbf{D}_{\mathrm{Qcoh}}(X)$ is the one with better properties. If we are willing to replace $\mathbf{D}(\mathrm{Qcoh} / X)$ by $\mathbf{D}_{\mathrm{Qcoh}}(X)$ in what follows then many of the results, quite possibly all, undoubtedly generalize to schemes which are only quasiseparated. But I have not carefully checked the details.

When $X$ is noetherian we will also consider the category $\mathbf{D}^{b}(\mathrm{Coh} / X)$; this is our notation for the derived category of bounded complexes of coherent sheaves. Note that coherent sheaves make sense on a noetherian scheme. The natural functor $\mathbf{D}^{b}(\mathrm{Coh} / X) \longrightarrow \mathbf{D}(\mathrm{Q} \operatorname{coh} / X)$ is known to be fully faithful. The essential image is the category which is sometimes 
denoted $\mathbf{D}_{\text {Coh }}^{b}(\mathrm{Qcoh} / X)$; the objects are (unbounded) complexes of quasicoherent sheaves, whose cohomology is bounded and coherent. Since the categories $\mathbf{D}^{b}(\mathrm{Coh} / X)$ and $\mathbf{D}_{\mathrm{Coh}}^{b}(\mathrm{Q} c o h / X)$ are equivalent we will freely confuse them, and use the shorter symbol $\mathbf{D}^{b}(\mathrm{Coh} / X)$ to stand for both.

Given two objects $\mathcal{E}$ and $\mathcal{F}$ in $\mathbf{D}(\mathrm{Qcoh} / X)$, we may form the derived tensor product $\mathcal{E}^{\mathbf{L}} \otimes \mathcal{F}$. It is easy to show that the derived tensor is a triangulated functor in each of the variables $\mathcal{E}$ and $\mathcal{F}$, and respects coproducts in either variable. If we fix $\mathcal{F}$ and consider the functor $\mathcal{E} \mapsto \mathcal{E}^{\mathbf{L}} \otimes \mathcal{F}$, we have a triangulated functor respecting coproducts, which we write

$$
-{ }^{\mathbf{L}} \otimes \mathcal{F}: \mathbf{D}(\mathrm{Q} \operatorname{coh} / X) \longrightarrow \mathbf{D}(\mathrm{Q} \operatorname{coh} / X) .
$$

We know that the category $\mathbf{D}(\mathrm{Qcoh} / X)$ is compactly generated; see $[51$, Proposition 2.5]. From [51, Theorem 4.1] it follows that the coproductpreserving triangulated functor $-\mathbf{L}_{\otimes} \mathcal{F}$ must have a right adjoint; there is a functor $\mathbf{R} \mathcal{H} \operatorname{lom}(\mathcal{F},-): \mathbf{D}(\mathrm{Q} \operatorname{coh} / X) \longrightarrow \mathbf{D}(\mathrm{Q} \operatorname{coh} / X)$, with

$$
\operatorname{Hom}\left(\mathcal{E}^{\mathbf{L}} \otimes \mathcal{F}, \mathcal{G}\right) \cong \operatorname{Hom}(\mathcal{E}, \mathbf{R} \mathcal{H} \operatorname{Com}(\mathcal{F}, \mathcal{G}))
$$

The category $\mathbf{D}(\mathrm{Qcoh} / X)$ becomes a symmetric, monoidal category, and both the tensor product and the internal Hom are triangulated functors in each of the two variables ${ }^{3}$.

Reminder 2.2. Next we remind the reader of a technical observation which may be found in [48, Lemma 6.8]. For this observation we need a little more notation. The scheme $X$ is still assumed quasicompact and separated, but now we wish to also consider a quasicompact open subset $U \subset X$. We will let $j: U \longrightarrow X$ stand for the inclusion. Because we now have two schemes, we distinguish $\mathbf{R} \mathcal{H}_{0} m_{U}(\mathcal{F}, \mathcal{G})$ from $\mathbf{R} \mathcal{H} \operatorname{Hom}_{X}(\mathcal{F}, \mathcal{G})$; if $\mathcal{F}, \mathcal{G}$ are objects of $\mathbf{D}(\mathrm{Qcoh} / U)$ then the first is the $\mathbf{R} \mathcal{H}$ om which makes sense, while if $\mathcal{F}, \mathcal{G}$ belong to $\mathbf{D}(\mathrm{Qcoh} / X)$, then the second is well-defined.

The remark we want is that, if $\mathcal{F} \in \mathbf{D}(\mathrm{Q} \operatorname{coh} / X)$ and $\mathcal{G} \in \mathbf{D}(\mathrm{Qcoh} / U)$, then there is a natural isomorphism

$$
\mathbf{R} \mathcal{H} \operatorname{oom}_{X}\left(\mathcal{F}, \mathbf{R} j_{*} \mathcal{G}\right) \cong \mathbf{R} j_{*} \mathbf{R} \mathcal{H} \operatorname{oom}_{U}\left(j^{*} \mathcal{F}, \mathcal{G}\right)
$$

\footnotetext{
${ }^{3}$ It is slightly subtle to show that the internal Hom is triangulated in the first variable. For details see Murfet's thesis [48, Theorem C.1]. There are also older proofs in the literature, for example $[41, \S 1.5 .3]$. Unlike the older arguments, the proof in [48] is in the spirit of this section; it appeals to the definition of the internal Hom as right adjoint to the tensor product, and develops the property of the tensor product that would suffice to formally deduce that the right adjoint must be triangulated in the first variable.
} 
The proof is easy; the result follows from the isomorphisms

$$
\begin{aligned}
\operatorname{Hom}_{X}\left(\mathcal{E}, \mathbf{R \mathcal { H }}_{X}\left(\mathcal{F}, \mathbf{R} j_{*} \mathcal{G}\right)\right) & \cong \operatorname{Hom}_{X}\left(\mathcal{E} \mathbf{L} \otimes \mathcal{F}, \mathbf{R} j_{*} \mathcal{G}\right) \\
& \cong \operatorname{Hom}_{U}\left(j^{*} \mathcal{E}, \mathbf{R} \mathcal{H} m_{U}\left(j^{*} \mathcal{F}, \mathcal{G}\right)\right) \\
& \cong \operatorname{Hom}_{X}\left(\mathcal{E}, \mathbf{R} j_{*} \mathbf{R} \mathcal{H} \operatorname{Hom}_{U}\left(j^{*} \mathcal{F}, \mathcal{G}\right)\right) .
\end{aligned}
$$

Remark 2.3. With the notation as in Reminder 2.2 we observe that, for any pair of objects $\mathcal{F}, \mathcal{G} \in \mathbf{D}(\mathrm{Qcoh} / X)$, there is always a map $\gamma(\mathcal{F}, \mathcal{G})$ : $j^{*} \mathbf{R} \mathcal{H} m_{X}(\mathcal{F}, \mathcal{G}) \longrightarrow \mathbf{R} \mathcal{H} \operatorname{Lom}_{U}\left(j^{*} \mathcal{F}, j^{*} \mathcal{G}\right)$. To construct it, consider the sequence of maps

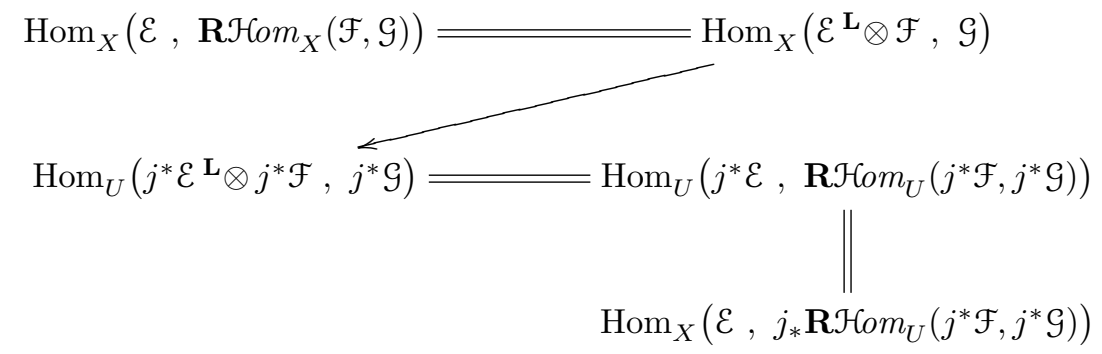

They are all natural in $\mathcal{E}$ and we deduce, in the category $\mathbf{D}(\mathrm{Qcoh} / X)$, a morphism $\mathbf{R} \mathcal{H} \operatorname{Com}_{X}(\mathcal{F}, \mathcal{G}) \longrightarrow j_{*} \mathbf{R} \mathcal{H} \operatorname{Com}_{U}\left(j^{*} \mathcal{F}, j^{*} \mathcal{G}\right)$. Adjunction gives us, in the category $\mathbf{D}(\mathrm{Qcoh} / U)$, the required map $\gamma(\mathcal{F}, \mathcal{G}): j^{*} \mathbf{R} \mathcal{H} m_{X}(\mathcal{F}, \mathcal{G}) \longrightarrow$ $\mathbf{R} \mathcal{H o m}_{U}\left(j^{*} \mathcal{F}, j^{*} \mathcal{G}\right)$.

The morphism $\gamma(\mathcal{F}, \mathcal{G})$ need not be an isomorphism. In general, all we know for certain is that it is natural in $\mathcal{F}$ and $\mathcal{G}$. There is, however, a useful special case in which we can show $\gamma(\mathcal{F}, \mathcal{G})$ to be an isomorphism.

Lemma 2.4. Assume that $U \subset X$ is a quasicompact open subset of a quasicompact, separated scheme $X$. Suppose $\mathcal{G} \in \mathbf{D}(\mathrm{Qcoh} / X)$ is any object, and let $\mathcal{F} \in \mathbf{D}(\mathrm{Qcoh} / X)$ satisfy one of the two possible conditions:

(i) $X$ is a noetherian scheme, and $\mathcal{F}$ belongs to $\mathbf{D}^{b}(\mathrm{Coh} / X) \subset \mathbf{D}(\mathrm{Qcoh} / X)$.

(ii) $X$ is general, but we assume $\mathcal{F}$ is a compact object in $\mathbf{D}(\mathrm{Qcoh} / X)$. $W$ remind the reader: the compact objects are the perfect complexes.

If either (i) or (ii) holds, then the morphism

$$
\gamma(\mathcal{F}, \mathcal{G}): j^{*} \mathbf{R} \mathcal{H} \operatorname{lom}_{X}(\mathcal{F}, \mathcal{G}) \longrightarrow \mathbf{R} \mathcal{H o m} m_{U}\left(j^{*} \mathcal{F}, j^{*} \mathcal{G}\right)
$$

of Remark 2.3 is an isomorphism.

Proof. Assume first that both $U$ and $X$ are affine. In that case we have rings $R$ and $S$ so that $X=\operatorname{Spec}(R)$ and $U=\operatorname{Spec}(S)$, and we know that the open immersion $j: U \longrightarrow X$ is flat, meaning that $S$ must be a flat $R$-algebra. The derived category $\mathbf{D}(\mathrm{Q} \operatorname{coh} / X)$ identifies with $\mathbf{D}(R-\mathrm{Mod})$, the derived category $\mathbf{D}(\mathrm{Q} c o h / U)$ is $\mathbf{D}(S-\mathrm{Mod})$, and the functor $j^{*}$ is nothing more than 
tensoring a chain complex of $R$-modules with $S$. The lemma is now an easy consequence of the observation that, if $M$ and $N$ are $R-$ modules with $M$ finitely presented, then the $S$-module homomorphism

$$
S \otimes_{R} \operatorname{Hom}_{R}(M, N) \longrightarrow \operatorname{Hom}_{R}\left(S \otimes_{R} M, S \otimes_{R} N\right)
$$

is an isomorphism.

Next we will see how to deduce the case where only $U$ is assumed affine. We note that, if we fix $\mathcal{F}$ and let $\mathcal{G}$ vary, the maps $\gamma(\mathcal{F}, \mathcal{G})$ assemble to a natural transformation between triangulated functors. To show that the maps $\gamma(\mathcal{F}, \mathcal{G})$ are isomorphisms, for fixed $\mathcal{F}$ and all $\mathcal{G}$, it therefore suffices to prove the special case where $\mathcal{G}$ belongs to some class of generators for $\mathbf{D}(\mathrm{Q} c o h / X)$. The class we will use is the objects $\left\{j_{V}^{X}\right\}_{*} \mathcal{G}=\mathbf{R}\left\{j_{V}^{X}\right\}_{*} \mathcal{G}$, where $j_{V}^{X}: V \longrightarrow X$ is the open immersion of an open affine subset $V \subset X$. Observe that, since $V \subset X$ is assumed affine, the functor $\left\{j_{V}^{X}\right\}_{*}$ is exact; hence $\left\{j_{V}^{X}\right\}_{*} \mathcal{G}=\mathbf{R}\left\{j_{V}^{X}\right\}_{*} \mathcal{G}$. See [48, Corollary 3.14] for the fact that these generate.

Because we now have several open subsets involved, we will adopt the convention that $j_{W_{1}}^{W_{2}}: W_{1} \longrightarrow W_{2}$ stands for the open immersion between two subsets $W_{1} \subset W_{2}$ of $X$.

The lemma now follows from the isomorphisms

$$
\begin{aligned}
\left\{j_{U}^{X}\right\}^{*} \mathbf{R} \mathcal{H o m}\left(\mathcal{F},\left\{j_{V}^{X}\right\}_{*} \mathcal{G}\right) & \cong\left\{j_{U}^{X}\right\}^{*}\left\{j_{V}^{X}\right\}_{*} \mathbf{R} \mathcal{H} \operatorname{Hom}\left(\left\{j_{V}^{X}\right\}^{*} \mathcal{F}, \mathcal{G}\right) \\
& \cong\left\{j_{U \cap V}^{U}\right\}_{*}\left\{j_{U \cap V}^{V}\right\}^{*} \mathbf{R} \mathcal{H o m}\left(\left\{j_{V}^{X}\right\}^{*} \mathcal{F}, \mathcal{G}\right) \\
& \cong\left\{j_{U \cap V}^{U}\right\}_{*} \mathbf{R} \mathcal{H o m}\left(\left\{j_{U \cap V}^{V}\right\}^{*}\left\{j_{V}^{X}\right\}^{*} \mathcal{F},\left\{j_{U \cap V}^{V}\right\}^{*} \mathcal{G}\right) \\
& \cong\left\{j_{U \cap V}^{U}\right\}_{*} \mathbf{R} \mathcal{H o m}\left(\left\{j_{U \cap V}^{U}\right\}^{*}\left\{j_{U}^{X}\right\}^{*} \mathcal{F},\left\{j_{U \cap V}^{V}\right\}^{*} \mathcal{G}\right) \\
& \cong \mathbf{R} \mathcal{H o m}\left(\left\{j_{U}^{X}\right\}^{*} \mathcal{F},\left\{j_{U \cap V}^{U}\right\}_{*}\left\{j_{U \cap V}^{V}\right\}^{*} \mathcal{G}\right) \\
& \cong \mathbf{R} \mathcal{H o m}\left(\left\{j_{U}^{X}\right\}^{*} \mathcal{F},\left\{j_{U}^{X}\right\}^{*}\left\{j_{V}^{X}\right\}_{*} \mathcal{G}\right)
\end{aligned}
$$

The first isomorphism is by Reminder 2.2, the second is by base change, the third is by the affine case which we already know, the fourth is trivial, the fifth is again by Reminder 2.2, and finally the sixth is yet another base change.

Finally we need to see how to deduce the general case, where neither $U$ nor $X$ need be affine. We wish to show that the map $\gamma(\mathcal{F}, \mathcal{G}): j^{*} \mathbf{R} \mathcal{H} \operatorname{lom}_{X}(\mathcal{F}, \mathcal{G})$ $\longrightarrow \mathbf{R} \mathcal{H o m}_{U}\left(j^{*} \mathcal{F}, j^{*} \mathcal{G}\right)$ is an isomorphism. The problem is local; it suffices to show that it restricts to an isomorphism on each affine open set $V \subset U$. Choose such an affine open set, and let $i: V \longrightarrow U$ be the inclusion. It suffices to show that the map

$$
i^{*} \gamma(\mathcal{F}, \mathcal{G}): i^{*} j^{*} \mathbf{R} \mathcal{H} \operatorname{Hom}_{X}(\mathcal{F}, \mathcal{G}) \longrightarrow i^{*} \mathbf{R} \mathcal{H} \operatorname{com}_{U}\left(j^{*} \mathcal{F}, j^{*} \mathcal{G}\right)
$$


is an isomorphism. But we already know that the inclusions $i: V \longrightarrow U$ and $j i: V \longrightarrow X$ satisfy the Lemma, since $V$ is affine. Hence both complexes are naturally isomorphic to $\mathbf{R} \mathcal{H}_{0} m_{V}\left(i^{*} j^{*} \mathcal{F}, i^{*} j^{*} \mathcal{G}\right)$.

Remark 2.5. Suppose $X$ is a quasicompact, separated scheme. Given two complexes $\mathcal{E}, \mathcal{F} \in \mathbf{D}(\mathrm{Q} \operatorname{coh} / X)$ we have a counit of adjunction $\varepsilon$ : $\mathcal{E}^{\mathbf{L}} \otimes \mathbf{R} \mathcal{H} \operatorname{lom}(\mathcal{E}, \mathcal{F}) \longrightarrow \mathcal{F}$. If we have two more complexes $\mathcal{E}^{\prime}, \mathcal{F}^{\prime} \in \mathbf{D}(\mathrm{Q} \operatorname{coh} / X)$ they also give a map $\varepsilon: \mathcal{E}^{\prime \mathbf{L}} \otimes \mathbf{R} \mathcal{H} \operatorname{Hom}\left(\mathcal{E}^{\prime}, \mathcal{F}^{\prime}\right) \longrightarrow \mathcal{F}^{\prime}$. Combining the two we obtain a single morphism

$$
\mathcal{E}^{\mathbf{L}} \otimes \mathbf{R} \mathcal{H} \operatorname{com}(\mathcal{E}, \mathcal{F}) \mathbf{L}_{\otimes} \mathcal{E}^{\prime \mathbf{L}} \otimes \mathbf{R} \mathcal{H} \operatorname{Hom}\left(\mathcal{E}^{\prime}, \mathcal{F}^{\prime}\right) \stackrel{\varepsilon^{\mathbf{L}} \otimes \varepsilon}{\longrightarrow} \mathcal{F}^{\mathbf{L}} \otimes \mathcal{F}^{\prime},
$$

and adjunction produces for us a map

$$
\mathbf{R} \mathcal{H} \operatorname{lom}(\mathcal{E}, \mathcal{F}) \mathbf{L}_{\otimes} \mathbf{R} \mathcal{H} \operatorname{lom}\left(\mathcal{E}^{\prime}, \mathcal{F}^{\prime}\right) \longrightarrow \mathbf{R} \mathcal{H} \operatorname{Com}\left(\mathcal{E}^{\mathbf{L}} \otimes \mathcal{E}^{\prime}, \mathcal{F}^{\mathbf{L}} \otimes \mathcal{F}^{\prime}\right) .
$$

The special case where $\mathcal{E}^{\prime}=\mathcal{O}_{X}$ will interest us particularly; in this case we have a morphism

$$
\mu\left(\mathcal{E}, \mathcal{F}, \mathcal{F}^{\prime}\right): \mathbf{R} \mathcal{H} \operatorname{lom}(\mathcal{E}, \mathcal{F})^{\mathbf{L}} \otimes \mathcal{F}^{\prime} \longrightarrow \mathbf{R} \mathcal{H} \operatorname{lom}\left(\mathcal{E}, \mathcal{F}^{\mathbf{L}} \otimes \mathcal{F}^{\prime}\right) .
$$

We prove:

Lemma 2.6. Let $X$ be a quasicompact, separated scheme. If $\mathcal{E} \in \mathbf{D}(\mathrm{Qcoh} / X)$ is a perfect complex, and $\mathcal{F}$ and $\mathcal{F}^{\prime}$ in $\mathbf{D}(\mathrm{Qcoh} / X)$ are arbitrary, then the morphism $\mu\left(\mathcal{E}, \mathcal{F}, \mathcal{F}^{\prime}\right)$ of Remark 2.5 is an isomorphism.

Proof. We have a globally defined morphism in $\mathbf{D}(\mathrm{Q} \operatorname{coh} / X)$ and wish to prove it an isomorphism; it suffices to show that, for any open affine subset $U \subset X$, the morphism $\mu\left(\mathcal{E}, \mathcal{F}, \mathcal{F}^{\prime}\right)$ restricts to an isomorphism on $U$. Let $j: U \longrightarrow X$ be the inclusion. Lemma 2.4 permits us to identify

$$
\begin{aligned}
& j^{*} \mathbf{R} \mathcal{H o m}(\mathcal{E}, \mathcal{F}) \cong \mathbf{R} \mathcal{H o m}\left(j^{*} \mathcal{E}, j^{*} \mathcal{F}\right), \\
& j^{*} \mathbf{R} \mathcal{H o m}\left(\mathcal{E}, \mathcal{F}^{\mathbf{L}_{\otimes}} \mathcal{F}^{\prime}\right) \cong \mathbf{R} \mathcal{H o m}\left(j^{*} \mathcal{E}, j^{*} \mathcal{F}^{\mathbf{L}_{\otimes}} j^{*} \mathcal{F}^{\prime}\right)
\end{aligned}
$$

and we conclude that $j^{*} \mu\left(\mathcal{E}, \mathcal{F}, \mathcal{F}^{\prime}\right)=\mu\left(j^{*} \mathcal{E}, j^{*} \mathcal{F}, j^{*} \mathcal{F}^{\prime}\right)$. We are therefore reduced to proving the Lemma in the case where $X$ is affine. Put $X=\operatorname{Spec}(R)$; then the category $\mathbf{D}(\mathrm{Q} \operatorname{coh} / X)$ identifies with $\mathbf{D}(R-$ Mod $)$, and under the identification the perfect complex $\mathcal{E}$ becomes a bounded complex of finitely generated projectives. Because $\mu\left(\mathcal{E}, \mathcal{F}, \mathcal{F}^{\prime}\right)$ is a natural tranformation of triangulated functors in $\mathcal{E}$, we easily reduce to the case where $\mathcal{E}$ is a single, finitely generated free module concentrated in degree 0 , and in this case the result is obvious.

Reminder 2.7. Consider the special case of Lemma 2.6, where we let $\mathcal{F}=\mathcal{O}_{X}$. From Lemma 2.6 we learn that, for every perfect complex $\mathcal{E}$ and for any object $\mathcal{F}^{\prime} \in \mathbf{D}(\mathrm{Qcoh} / X)$, the natural map

$$
\mathbf{R} \mathcal{H} o m\left(\mathcal{E}, \mathcal{O}_{X}\right) \mathbf{L} \otimes \mathcal{F}^{\prime} \longrightarrow \mathbf{R} \mathcal{H} \text { om }\left(\mathcal{E}, \mathcal{F}^{\prime}\right)
$$


is an isomorphism. This brings us to the well-explored realm of strongly dualizable objects. We remind the reader.

It is traditional to define $\mathcal{E}^{\vee}=\mathbf{R} \mathcal{H} \operatorname{Com}\left(\mathcal{E}, \mathcal{O}_{X}\right)$. The standard terminology is that $\mathcal{E}$ is strongly dualizable if the natural map $\mathcal{E}^{\vee} \mathbf{L} \otimes \mathcal{F}^{\prime} \longrightarrow \mathbf{R} \mathcal{H}$ om $\left(\mathcal{E}, \mathcal{F}^{\prime}\right)$ is an isomorphism; the previous paragraph taught us that all perfect complexes are strongly dualizable. From the literature on strongly dualizable objects we learn that

(i) If $\mathcal{E}$ is a strongly dualizable object then the natural map $\mathcal{E} \longrightarrow\left\{\mathcal{E}^{\vee}\right\}^{\vee}$ is an isomorphism. See [40, Proposition 1.3(i), p. 122].

(ii) If $\mathcal{E}$ is strongly dualizable then so is $\mathcal{E}^{\vee}$. See [40, Proposition 1.2, p. 121$]^{4}$.

(iii) Let $\mathcal{T}$ be a compactly generated triangulated category, with a symmetric tensor product compatible with the triangulated structure. Assume that the unit of the tensor is compact, and that all compact objects are strongly dualizable. For example $\mathcal{T}$ could be $\mathbf{D}(\mathrm{Q} \operatorname{coh} / X)$. If $\mathcal{E} \in \mathcal{T}$ is some object, then the following are equivalent:

(a) $\mathcal{E}$ is compact.

(b) $\mathcal{E}$ is strongly dualizable.

(c) Tensor product with $\mathcal{E}$ commutes with arbitrary products in $\mathcal{T}$; that is, the natural map

$$
\mathcal{E}^{\mathbf{L}} \otimes \prod_{\lambda \in \Lambda} t_{\lambda} \longrightarrow \prod_{\lambda \in \Lambda}\left(\mathcal{E}^{\mathbf{L}} \otimes t_{\lambda}\right)
$$

is an isomorphism for every set of objects $\left\{t_{\lambda}, \lambda \in \Lambda\right\}$.

The fact that (c) implies (a) seems new; in Theorem A.1 we will give a self-contained proof that (a), (b) and (c) are equivalent.

In subsequent sections, especially $\S 4$, we will feel free to use (i), (ii) and (iii) above.

Remark 2.8. The counit of adjunction gives a natural map

$$
\varepsilon: \mathcal{E}^{\mathbf{L}} \otimes \mathbf{R} \mathcal{H} \circ o m(\mathcal{E}, \mathcal{F}) \longrightarrow \mathcal{F} .
$$

Combining two such, we deduce a composite

$$
\mathcal{E}^{\mathbf{L}} \otimes \mathbf{R} \mathcal{H} \operatorname{om}(\mathcal{E}, \mathcal{F}) \mathbf{L}_{\otimes} \mathbf{R} \mathcal{H o m}(\mathcal{F}, \mathcal{G}) \stackrel{\varepsilon \otimes 1}{\longrightarrow} \mathcal{F} \mathbf{L}_{\otimes} \mathbf{R} \mathcal{H} \operatorname{Hom}(\mathcal{F}, \mathcal{G}) \stackrel{\varepsilon}{\longrightarrow} \mathcal{G} .
$$

By adjunction this corresponds to a morphism

$$
\nu(\mathcal{E}, \mathcal{F}, \mathcal{G}): \mathcal{E}^{\mathbf{L}} \otimes \mathbf{R} \mathcal{H} \operatorname{Hom}(\mathcal{F}, \mathcal{G}) \longrightarrow \mathbf{R} \mathcal{H o m}(\mathbf{R} \mathcal{H} \operatorname{com}(\mathcal{E}, \mathcal{F}), \mathcal{G}) .
$$

\footnotetext{
${ }^{4}$ The objects we call "strongly dualizable" are labeled "finite" in [40]. In the second last paragraph on $[40$, p. 120] the authors refer to [10] for the older terminolgy, which is the one in current use. Similar notions appeared even earlier, under different names; see the " $\otimes$-categories rigides" of $[57, \S$ I.5, p. 78], or the "compact closed categories" of $[33$, pp. 102-103].
} 
We will find useful the next little lemma; note that, from now until the end of the section, all our schemes are assumed noetherian.

Lemma 2.9. Let $X$ be a noetherian, separated scheme. Let the notation be as above, with the objects $\mathcal{F}, \mathcal{G} \in \mathbf{D}(\mathrm{Q} \operatorname{coh} / X)$ fixed. Suppose that, for all $\varepsilon \in \mathbf{D}^{b}(\mathrm{Coh} / X)$,

(i) The complex $\mathbf{R} \mathcal{H} \operatorname{com}(\mathcal{E}, \mathcal{F})$ belongs to $\mathbf{D}^{b}(\mathrm{Coh} / X) \subset \mathbf{D}(\mathrm{Qcoh} / X)$. The case $\mathcal{E}=\mathcal{O}_{X}$ tells us that $\mathcal{F} \in \mathbf{D}^{b}(\mathrm{Coh} / X)$.

(ii) The complex $\mathbf{R} \mathcal{H} \operatorname{Com}(\mathbf{R} \mathcal{H o m}(\mathcal{E}, \mathcal{F}), \mathcal{G})$ belongs to $\mathbf{D}^{-}(\mathrm{Qcoh} / X)$.

Then, for all $\mathcal{E} \in \mathbf{D}^{b}(\mathrm{Coh} / X)$, the map $\nu(\mathcal{E}, \mathcal{F}, \mathcal{G})$ is an isomorphism.

Proof. We have a globally defined morphism in the derived category, and we wish to prove it a homology isomorphism. The question is local; it suffices to show that the restriction of $\nu(\mathcal{E}, \mathcal{F}, \mathcal{G})$ to every open affine subset $U \subset X$ is a homology isomorphism. Choose any open affine $U \subset X$, and let $j: U \longrightarrow X$ be the inclusion. We propose to show that the map

$j^{*} \nu(\mathcal{E}, \mathcal{F}, \mathcal{G}): j^{*} \mathcal{E}^{\mathbf{L}} \otimes j^{*} \mathbf{R} \mathcal{H} \operatorname{lom}(\mathcal{F}, \mathcal{G}) \longrightarrow j^{*} \mathbf{R} \mathcal{H} \operatorname{com}(\mathbf{R} \mathcal{H} \operatorname{com}(\mathcal{E}, \mathcal{F}), \mathcal{G})$. is an isomorphism in $\mathbf{D}(\mathrm{Qcoh} / U)$.

At this point we use hypothesis (i) and Lemma 2.4. Because $\mathcal{F} \in$ $\mathbf{D}^{b}(\mathrm{Coh} / X)$, we have that $j^{*} \mathbf{R} \mathcal{H} \operatorname{Hom}(\mathcal{F}, \mathcal{G})=\mathbf{R} \mathcal{H o m}\left(j^{*} \mathcal{F}, j^{*} \mathcal{G}\right)$, because $\varepsilon \in$ $\mathbf{D}^{b}(\mathrm{Coh} / X)$ we know that $j^{*} \mathbf{R} \mathcal{H o m}(\mathcal{E}, \mathcal{F})=\mathbf{R} \mathcal{H} \operatorname{Com}\left(j^{*} \mathcal{E}, j^{*} \mathcal{F}\right)$, and because $\mathbf{R} \mathcal{H o m}(\mathcal{E}, \mathcal{F}) \in \mathbf{D}^{b}(\mathrm{Coh} / X)$ we conclude that

$$
\begin{aligned}
j^{*} \mathbf{R} \mathcal{H o m}(\mathbf{R} \mathcal{H o m}(\mathcal{E}, \mathcal{F}), \mathcal{G}) & =\mathbf{R} \mathcal{H o m}\left(j^{*} \mathbf{R} \mathcal{H o m}(\mathcal{E}, \mathcal{F}), j^{*} \mathcal{G}\right) \\
& =\mathbf{R} \mathcal{H o m}\left(\mathbf{R} \mathcal{H} \operatorname{Com}\left(j^{*} \mathcal{E}, j^{*} \mathcal{F}\right), j^{*} \mathcal{G}\right) .
\end{aligned}
$$

We are therefore reduced to showing that the map

$\nu\left(j^{*} \mathcal{E}, j^{*} \mathcal{F}, j^{*} \mathcal{G}\right): j^{*} \mathcal{E} \mathbf{L}_{\otimes} \mathbf{R} \mathcal{H o m}\left(j^{*} \mathcal{F}, j^{*} \mathcal{G}\right) \rightarrow \mathbf{R} \mathcal{H} \operatorname{lom}\left(\mathbf{R} \mathcal{H} o m\left(j^{*} \mathcal{E}, j^{*} \mathcal{F}\right), j^{*} \mathcal{G}\right)$

is an isomorphism in $\mathbf{D}(\mathrm{Qcoh} / U)$; in other words it suffices to prove the lemma in the special case where $X=U$ is affine.

Assume therefore that $X$ is affine. Then $X=\operatorname{Spec}(R)$ for a noetherian ring $R$, and $\mathbf{D}(\mathrm{Q} \operatorname{coh} / X)$ is identified with $\mathbf{D}(R$-Mod $)$. The complex $\mathcal{E}$ becomes a bounded complex of finitely generated $R$-modules, and, because $\nu(\mathcal{E}, \mathcal{F}, \mathcal{G})$ is a natural transformation between triangulated functors in $\mathcal{E}$, it suffices to prove $\nu(\mathcal{E}, \mathcal{F}, \mathcal{G})$ to be an isomorphism in the special case where $\mathcal{E}$ is a single, finitely generated module concentrated in degree 0 . Next observe that, for any $\mathcal{V}, \mathcal{W} \in \mathbf{D}(R-\operatorname{Mod})$, we may construct $\mathbf{R H o m}(\mathcal{V}, \mathcal{W})$ by first replacing $\mathcal{W}$ by a $K$-injective resolution consisting of injective objects (see, for example, [4, Applications 2.4 and 2.4']), and then computing the ordinary Hom-complex. For the problem at hand, let us replace $\mathcal{F}$ and $\mathcal{G}$ by $K$-injective resolutions of injectives. All the complexes $\operatorname{RHom}(\mathcal{V}, \mathcal{W})$, 
on both sides of the map $\nu(\mathcal{E}, \mathcal{F}, \mathcal{G})$, simplify to $\operatorname{Hom}(\mathcal{V}, \mathcal{W})$. Note also that, because $R$ is noetherian, we know that $\operatorname{Hom}(\mathcal{F}, \mathcal{G})$ is a complex of flat modules; for any two injective modules $I$ and $J$ we have that $\operatorname{Hom}(I, J)$ is flat, and products of flat modules are flat. However, we do not yet know that the complex $\operatorname{Hom}(\mathcal{F}, \mathcal{G})$ is $K$-flat; in other words there remains a derived tensor product which we will eventually need to handle. We must show that the morphism

$$
\nu(\mathcal{E}, \mathcal{F}, \mathcal{G}): \mathcal{E}^{\mathbf{L}} \otimes \operatorname{Hom}(\mathcal{F}, \mathcal{G}) \longrightarrow \operatorname{Hom}(\operatorname{Hom}(\mathcal{E}, \mathcal{F}), \mathcal{G})
$$

is an isomorphism in $\mathbf{D}(R-$ Mod $)$.

With our choices of complexes, that is where $\mathcal{F}$ and $\mathcal{G}$ have been replaced by $K$-injective resolutions by injectives and where $\mathcal{E}$ is a single, finitely generated module in degree 0 , we obtain a chain map of chain complexes

$$
\alpha: \mathcal{E} \otimes \operatorname{Hom}(\mathcal{F}, \mathcal{G}) \longrightarrow \operatorname{Hom}(\operatorname{Hom}(\mathcal{E}, \mathcal{F}), \mathcal{G}) \text {. }
$$

Do not confuse $\alpha$ with the map $\nu(\mathcal{E}, \mathcal{F}, \mathcal{G})$; we are not yet asserting that they agree. We will now prove that the morphism $\alpha$ is an isomorphism of chain complexes. To see this, choose a finite presentation of the module $\mathcal{E}$. That is, choose an exact sequence

$$
R^{m} \longrightarrow R^{n} \longrightarrow \mathcal{E} \longrightarrow 0
$$

We deduce a diagram of chain maps of chain complexes

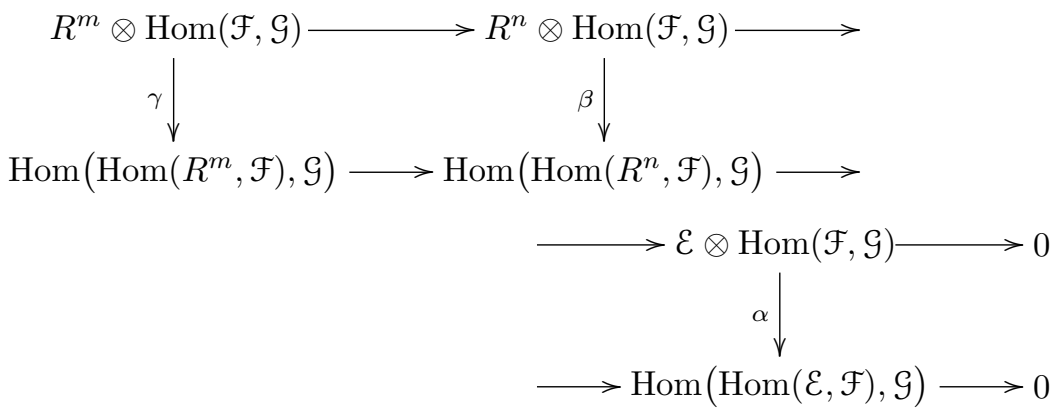

The rows are clearly exact, we know that $\beta$ and $\gamma$ are isomorphisms, and hence so is $\alpha$. We have computed the complex $\operatorname{Hom}(\operatorname{Hom}(\mathcal{E}, \mathcal{F}), \mathcal{G})$, and the lemma now reduces to showing that the natural map

$$
\mathcal{E}^{\mathbf{L}} \otimes \operatorname{Hom}(\mathcal{F}, \mathcal{G}) \longrightarrow \mathcal{E} \otimes \operatorname{Hom}(\mathcal{F}, \mathcal{G})
$$

is a homology isomorphism. We must show that the derived tensor product agrees with the simple-minded tensor product. It certainly suffices to prove that $\operatorname{Hom}(\mathcal{F}, \mathcal{G})$ is $K$-flat. 
Now we use Hypothesis (ii) of the Lemma. We are given that, for any finite $R$-module $\mathcal{E}$, the complex

$$
\mathcal{E} \otimes \operatorname{Hom}(\mathcal{F}, \mathcal{G}) \cong \operatorname{Hom}(\operatorname{Hom}(\mathcal{E}, \mathcal{F}), \mathcal{G})
$$

belongs to $\mathbf{D}^{-}(R-$ Mod $)$; its cohomology is bounded above. Lemma 2.9 therefore follows from the following technical lemma.

Lemma 2.10. Let $R$ be a commutative, noetherian ring, and let $\mathcal{H}$ be a complex of flat $R$-modules. Suppose that, for any finite $R$-module $M$, we have

(i) $H^{i}(M \otimes \mathcal{H})=0$ for $i \gg 0$. More precisely: for every $M$ there exists an integer $N=N(M)$, which may depend on $M$, so that $i>N(M)$ implies $H^{i}(M \otimes \mathcal{H})=0$.

Then the complex $\mathcal{H}$ is $K$-flat.

Remark 2.11. The proof we give for Lemma 2.10 is due to Avramov and Iyengar; it is a simplified version of my original, clumsy argument. The remainder of the section is devoted to the proof; the reader may want to skip ahead to $\S 3$. In subsequent sections we will not make use of any of the ideas in the proof.

Proof. We need to show that $\mathcal{H}$ is $K$-flat. We remind the reader: this means that $X \otimes \mathcal{H}$ must be shown acyclic for every acyclic complex $X$ of $R$-modules. We will break up the proof into steps. We begin with

Step 1. It suffices to prove the Lemma under the extra hypotheses that $\mathcal{H}$ is acyclic.

Proof. Suppose $\mathcal{H}$ is an object in $\mathbf{K}(R$-Flat) satisfying the hypotheses of the Lemma; $\mathcal{H}$ does not have to be acyclic. Let us apply the hypothesis (i) of the Lemma in the special case $M=R$; we have a finite module $R$, and (i) tells us that $H^{i}(\mathcal{H})=H^{i}(R \otimes \mathcal{H})$ vanishes for $i \gg 0$. Choose a $K$-projective resolution of $\mathcal{G} \longrightarrow \mathcal{H}$. The map $\mathcal{G} \longrightarrow \mathcal{H}$ is a quasi-isomorphism, and the complex $\mathcal{G}$ may be chosen to belong to $\mathbf{K}^{-}(R-\operatorname{Proj})$; that is we can take $\mathcal{G}$ to be a bounded above complex of projective $R$-modules. Complete the morphism $\mathcal{G} \longrightarrow \mathcal{H}$ to a distinguished triangle in $\mathbf{K}(R$-Flat $)$

$$
\mathcal{G} \longrightarrow \mathcal{H} \longrightarrow \mathcal{H}^{\prime} \longrightarrow \Sigma \mathcal{G} ;
$$

this produces an acyclic complex $\mathcal{H}^{\prime}$ of flat $R$-modules. We furthermore know that $\mathcal{G}$, being a bounded above complex of projectives, is definitely $K$-flat, and it therefore suffices to show that $\mathcal{H}^{\prime}$ is $K$-flat. The hypothesis (i) of the Lemma holds for $\mathcal{H}$ by assumption, and for $\mathcal{G}$ because it is a bounded above complex. Hence $\mathcal{H}^{\prime}$ also satisfies the hypotheses of the 
Lemma. Replacing $\mathcal{H}$ by $\mathcal{H}^{\prime}$, we are reduced to proving the Lemma when the object $\mathcal{H}$ is acyclic.

Strategy: We wish to show that $X \otimes \mathcal{H}$ is acyclic when $X$ is. It suffices to show the acyclicity of the localizations $(X \otimes \mathcal{H})_{\mathfrak{p}}=X \otimes \mathcal{H}_{\mathfrak{p}}$ for every prime ideal $\mathfrak{p} \subset R$. That is, it suffices to prove that each $\mathcal{H}_{\mathfrak{p}}$ is $K$-flat. We will prove this by induction on the height of the prime ideal $\mathfrak{p}$. To give away our strategy even more completely, we will consider the two conditions

(ii) For every prime ideal of height $\leq n$ the complex $\mathcal{H}_{p}$ is $K$-flat.

(iii) For every prime ideal $\mathfrak{p} \subset R$ of height $\leq n$, for every finite $R$-module $M$, and for every integer $i$, we have that $H^{i}\left(M \otimes \mathcal{H}_{p}\right)$ is $\mathfrak{p}$-torsion.

Our induction will be to show that if (ii) is true for $n$ then (iii) is true for $n+1$, and if (iii) is true for $n$ then (ii) is true for $n$. Note that (iii) is obviously true for $n=0$; this starts the induction.

Step 2. If (ii) is true for $n$ then (iii) is true for $n+1$.

Proof. Let $\mathfrak{p}$ be a prime ideal of height $n+1$ and let $M$ be a finite $R$-module. We wish to show that the $R_{\mathfrak{p}}$-modules $H^{i}\left(M \otimes \mathcal{H}_{\mathfrak{p}}\right)$ are all $\mathfrak{p}$-torsion; it suffices to show that their localizations at prime ideals $\mathfrak{q}$ vanish, as long as $\mathfrak{q}$ is properly contained in $\mathfrak{p}$. Now localization is flat, and hence

$$
\left[H^{i}\left(M \otimes \mathcal{H}_{\mathfrak{p}}\right)\right]_{\mathfrak{q}} \cong H^{i}\left(M \otimes \mathcal{H}_{\mathfrak{q}}\right) ;
$$

it suffices to show that each complex $M \otimes \mathcal{H}_{\mathfrak{q}}$ is acyclic. Since $\mathfrak{q}$ is properly contained in $\mathfrak{p}$ it has height $\leq n$, and, because (ii) is true for $n$, we know that $\mathcal{H}_{\mathfrak{q}}$ is $K$-flat. This makes it a $K$-flat, acyclic complex. The acyclicity says that, in the derived category $\mathbf{D}(R-\mathrm{Mod})$, the complex $\mathcal{H}_{q}$ is isomorphic to zero. The fact that it is $K$-flat permits us to compute, in $\mathbf{D}(R$-Mod $)$,

$$
M \otimes \mathcal{H}_{\mathfrak{q}} \cong M^{\mathbf{L}} \otimes \mathcal{H}_{\mathfrak{q}} \cong M^{\mathbf{L}} \otimes 0=0 .
$$

This gives the acyclicity of $M \otimes \mathcal{H}_{\mathfrak{q}}$.

Notation 2.12. It remains to prove that if (iii) is true for $n$ then so is (ii). We fix a prime ideal $\mathfrak{p}$ of height $n$; we want to prove that $\mathcal{H}_{\mathfrak{p}}$ is $K$-flat. Let us simplify the notation a little. Observe first that $X \otimes_{R} \mathcal{H}_{p} \cong X_{\mathfrak{p}} \otimes_{R_{\mathfrak{p}}} \mathcal{H}_{p}$; replacing $R$ by $R_{\mathfrak{p}}$ we may assume $R$ is a local ring of height $n$, with maximal ideal $\mathfrak{p}$ and residue field $k=R / \mathfrak{p}$. Let us further replace $\mathcal{H}$ by the chain complex $\mathcal{H}_{\mathfrak{p}}$. What we know so far is:

(iv) For every finite $R$-module $M$ we have $H^{i}(M \otimes \mathcal{H})=0$ if $i \gg 0$; this comes from hypothesis (i) of the Lemma.

(v) The complex $\mathcal{H}$ is an acyclic complex of flat $R$-modules; that comes from Step 1. 
(vi) For all finite $R$-modules $M$ and for all integers $i$ we have that $H^{i}(M \otimes$ $\mathcal{H})$ is $\mathfrak{p}$-torsion. This is because we are assuming that (iii) holds for $n$.

We want to prove that, under these conditions, $\mathcal{H}$ must be $K$-flat.

Step 3. With the conventions of Notation 2.12, there exists an integer $\ell$ so that $H^{i}(M \otimes \mathcal{H})$ vanishes for all finite $R$-modules $M$ and for all $i>\ell$.

Proof. The proof will appeal to the following observation: $\mathcal{H}$ is a complex of flat modules, and therefore any short exact sequence $0 \longrightarrow M^{\prime} \longrightarrow M \longrightarrow$ $M^{\prime \prime} \longrightarrow 0$ of $R$-modules induces a short exact sequence of chain complexes

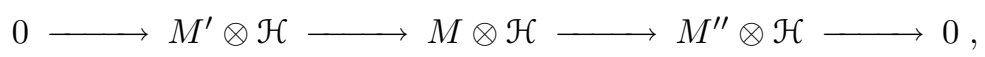

hence a long exact sequence in cohomology.

By (iv) we know that $H^{i}(k \otimes \mathcal{H})$ vanishes for $i \gg 0$. Shifting $\mathcal{H}$ if necessary, we may suppose that $H^{i}(k \otimes \mathcal{H})$ vanishes for $i>0$. If $M$ is a finite $R$-module with $\operatorname{dim}(M)=0$, then $M$ has a finite filtration with subquotients isomorphic to $k$. From the exact sequences in cohomology of the previous paragraph we immediately learn that $H^{i}(M \otimes \mathcal{H})$ must also vanish whenever $i>0$.

We will prove, by induction on $m=\operatorname{dim}(M)$, that $H^{i}(M \otimes \mathcal{H})$ vanishes if $i>\operatorname{dim}(M)$. The previous paragraph proved the case $m=0$. Since every module has dimension $\operatorname{dim}(M) \leq \operatorname{dim}(R)=n$, we will conclude that $H^{i}(M \otimes \mathcal{H})=0$ for any finite module $M$ and all $i>n$; that is Step 3 will immediately follow.

Suppose $m$ is an integer $\geq 0$, and assume we know the vanishing of $H^{i}(N \otimes \mathcal{H})$ provided $\operatorname{dim}(N) \leq m$ and $i>\operatorname{dim}(N)$. Let $M$ be a finite $R$-module with $\operatorname{dim}(M)=m+1$. Let $\Gamma_{\mathfrak{p}}(M)$ be the $\mathfrak{p}$-torsion submodule of $M$. We have a short exact sequence of $R$-modules

$$
0 \longrightarrow \Gamma_{\mathfrak{p}} M \longrightarrow M / \Gamma_{\mathfrak{p}} M \longrightarrow 0 \text {. }
$$

Since the module $\Gamma_{\mathfrak{p}} M$ is zero-dimensional we know the vanishing of $H^{i}\left(\Gamma_{\mathfrak{p}} M \otimes \mathcal{H}\right)$ for all $i>0$; this means that the map $H^{i}(M \otimes \mathcal{H}) \longrightarrow$ $H^{i}\left(\left[M / \Gamma_{\mathfrak{p}} M\right] \otimes \mathcal{H}\right)$ is an isomorphism for $i>0$. Replacing $M$ by $M / \Gamma_{\mathfrak{p}} M$ we may assume that $M$ has no $\mathfrak{p}$-torsion. We may choose an element $x \in \mathfrak{p}$ which is not a zero-divisor on $M$. Consider the exact sequence

$$
0 \longrightarrow M \stackrel{x}{\longrightarrow} M \longrightarrow M / x M \longrightarrow 0 \text {; }
$$

the long exact sequence in cohomology tells us the exactness of

$$
H^{i}([M / x M] \otimes \mathcal{H}) \longrightarrow H^{i+1}(M \otimes \mathcal{H}) \stackrel{x}{\longrightarrow} H^{i+1}(M \otimes \mathcal{H}) .
$$


The module $M / x M$ has dimension $m$, and the inductive hypothesis establishes the vanishing of $H^{i}([M / x M] \otimes \mathcal{H})$ if $i>m$. The exact sequence implies that multiplication by $x \in \mathfrak{p}$ is injective on $H^{i}(M \otimes \mathcal{H})$ when $i \geq m+1$. But (vi) informs us that the module $H^{i}(M \otimes \mathcal{H})$ is $\mathfrak{p}$-torsion, and hence must vanish.

Step 4. With the conventions of Notation 2.12, the complex $\mathcal{H}$ is $K$-flat.

Proof. Let the chain complex $\mathcal{H}$ be written as

$$
\cdots \longrightarrow \mathcal{H}^{i-2} \longrightarrow \mathcal{H}^{i-1} \longrightarrow \mathcal{H}^{i} \longrightarrow \mathcal{H}^{i+1} \longrightarrow \mathcal{H}^{i+2} \longrightarrow \cdots
$$

For each $i \in \mathbb{Z}$ let $I^{i}$ be the image of the homomorphism $\mathcal{H}^{i} \longrightarrow \mathcal{H}^{i+1}$. The acyclicity of $\mathcal{H}$ tells us that the sequence

$$
\mathcal{H}^{i-2} \longrightarrow \mathcal{H}^{i-1} \longrightarrow \mathcal{H}^{i} \longrightarrow I^{i} \longrightarrow 0
$$

is exact; it is the beginning of a flat resolution for the module $I^{i}$. Now let $M$ be any finite $R$-module, and consider the sequence

$M \otimes \mathcal{H}^{i-2} \longrightarrow M \otimes \mathcal{H}^{i-1} \longrightarrow M \otimes \mathcal{H}^{i} \longrightarrow M \otimes I^{i} \longrightarrow 0$.

In this sequence, the part

$$
M \otimes \mathcal{H}^{i-1} \longrightarrow M \otimes \mathcal{H}^{i} \longrightarrow M \otimes I^{i} \longrightarrow 0
$$

must be exact, simply from the right exactness of the tensor product. If $i>\ell+1$, with $\ell$ as in Step 3, then the bit

$$
M \otimes \mathcal{H}^{i-2} \longrightarrow M \otimes \mathcal{H}^{i-1} \longrightarrow M \otimes \mathcal{H}^{i}
$$

must also be exact, because $H^{i-1}(M \otimes \mathcal{H})=0$ for $i-1>\ell$. We conclude that, as long as $i>\ell+1$, the torsion group $\operatorname{Tor}_{1}^{R}\left(M, I^{i}\right)$ vanishes for all finite modules $M$. That is, $I^{i}$ is flat if $i>\ell+1$.

Now consider the short exact sequences

$$
0 \longrightarrow I^{i-1} \longrightarrow \mathcal{H}^{i} \longrightarrow I^{i} \longrightarrow 0 \text {. }
$$

We know that $\mathcal{H}^{i}$ is flat for all $i$. If $I^{i}$ is flat, the sequence tells us that so is $I^{i-1}$. We know that, for sufficiently large $i$, the modules $I^{i}$ are flat, and by descending induction we deduce the flatness of $I^{i}$ for every integer $i$. The short exact sequence

$$
0 \longrightarrow I^{i-1} \longrightarrow \mathcal{H}^{i} \longrightarrow I^{i} \longrightarrow 0
$$

is therefore a sequence of flat modules, and hence the sequences

$$
0 \longrightarrow M \otimes I^{i-1} \longrightarrow M \otimes \mathcal{H}^{i} \longrightarrow M \otimes I^{i} \longrightarrow 0
$$

are all exact. Piecing them all together, we conclude that the sequence

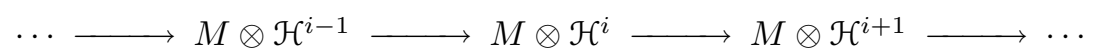


is exact. That is the complex $M \otimes \mathcal{H}$ is acyclic.

We now know that $M \otimes \mathcal{H}$ is acyclic for any finite module $M$. It is standard that $X \otimes \mathcal{H}$ must then be acyclic for any chain complex $X$ of $R$-modules; see, for example, [53, Corollary 8.4, (ii) $\Longleftrightarrow$ (iii)]. In particular $X \otimes \mathcal{H}$ is acyclic when $X$ is, that is $\mathcal{H}$ is $K$-flat.

\section{DuALizing COMPLEXes}

Throughout this section we continue to assume, as in the second half of $\S 2$, that $X$ is a noetherian, separated scheme. In $\S 2$ we prepared the ground by setting up some technical apparatus, and now we are ready to treat dualizing complexes. Let us first remind the reader of the definition.

Definition 3.1. Suppose, as agreed, that $X$ is a noetherian, separated scheme. A dualizing complex for $X$ is an object $\mathcal{J} \in \mathbf{D}^{b}(\mathrm{Coh} / X)$ so that the functor

$$
\mathcal{E} \mapsto \mathbf{R} \mathcal{H} \operatorname{Hom}(\mathcal{E}, \mathcal{J})
$$

gives an equivalence

$$
\mathbf{R} \mathcal{H o m}(-, \mathcal{J}): \mathbf{D}^{b}(\mathrm{Coh} / X)^{\text {op }} \longrightarrow \mathbf{D}^{b}(\mathrm{Coh} / X) .
$$

Remark 3.2. Perhaps we should remind the reader: when $\mathcal{E}$ and $\mathcal{J}$ are two objects of $\mathbf{D}^{b}(\mathrm{Coh} / X)$, the complex $\mathbf{R} \mathcal{H}$ om $(\mathcal{E}, \mathcal{J})$ has no obligation to belong to $\mathbf{D}^{b}(\mathrm{Coh} / X)$; there is no reason to expect its cohomology to be bounded above. It might help to recall the special case when $X=\operatorname{Spec}(R)$ is affine, and $\mathbf{D}^{b}(\mathrm{Coh} / X)$ reduces to $\mathbf{D}(R$-mod). Consider two finite $R-$ modules $M$ and $N$; they are objects of $\mathbf{D}(R-\bmod )$, and $\mathbf{R} \mathcal{H o m}(M, N)$ is a chain complex whose cohomology is $\operatorname{Ext}^{n}(M, N)$. It is perfectly possible to have $\operatorname{Ext}^{n}(M, N) \neq 0$ for infinitely many $n$.

As the above example illustrates it is a restriction on $\mathcal{J}$ to demand that, for every $\mathcal{E}$, the object $\mathbf{R H o m}(\mathcal{E}, \mathcal{J})$ be isomorphic to an object in $\mathbf{D}^{b}(\mathrm{Coh} / X)$. It is a severe restriction to further insist that the functor

$$
\mathbf{R H o m}(-, \mathcal{J}): \mathbf{D}^{b}(\mathrm{Coh} / X)^{\text {op }} \longrightarrow \mathbf{D}^{b}(\mathrm{Coh} / X)
$$

be an equivalence. In this section we will study some of the consequences.

Remark 3.3. The experts will notice that Definition 3.1 is unorthodox. It is customary to impose on $\mathcal{J}$ the technical condition that it have a finite injective resolution; that is, one traditionally assumes a dualizing complex $\mathcal{J}$ to be quasi-isomorphic to a bounded complex of injectives.

The whole thrust of this manuscript is that by now, more than forty years after derived categories were introduced, we have learnt enough about unbounded complexes to be able to handle them without trembling. It would be wimpy to assume boundedness, and we have decided to be tough. 
But the reader should be warned that, as a result of our decision to take the macho approach, Definition 3.1 is a little non-standard, as is what will now follow. What we will do amounts to proving that the usual results can be obtained without the boundedness hypothesis. And the reason for $\S 2$ was to provide us with the technical lemmas we will need.

Remark 3.4. Next observe that, for any object $\mathcal{G} \in \mathbf{D}(\mathrm{Qcoh} / X)$, the functor $\mathbf{R} \mathcal{H} \operatorname{com}(-, \mathcal{G}): \mathbf{D}(\mathrm{Q} \operatorname{coh} / X)^{\text {op }} \longrightarrow \mathbf{D}(\mathrm{Q} \operatorname{coh} / X)$ is its own left adjoint; this comes from the isomorphisms

$$
\begin{aligned}
\operatorname{Hom}(\mathcal{E}, \mathbf{R} \mathcal{H o m}(\mathcal{F}, \mathcal{G})) & \cong \operatorname{Hom}\left(\mathcal{E}^{\mathbf{L}} \otimes \mathcal{F}, \mathcal{G}\right) \\
& \cong \operatorname{Hom}(\mathcal{F}, \mathbf{R} \mathcal{H} \text { om }(\mathcal{E}, \mathcal{G})) .
\end{aligned}
$$

Furthermore, the unit and counit of this adjunction are the same morphism; they are both the natural map

$$
\mathcal{E} \longrightarrow \mathbf{R H o m}(\mathbf{R H o m}(\mathcal{E}, \mathcal{G}), \mathcal{G}) \text {. }
$$

If $\mathcal{G}$ is carefully chosen, so that $\mathbf{R} \mathcal{H} \operatorname{Com}(-, \mathcal{G})$ takes objects in $\mathbf{D}^{b}(\mathrm{Coh} / X)$ to objects in $\mathbf{D}^{b}(\mathrm{Coh} / X)$, then the adjunction restricts from the large category to the subcategory; that is

$$
\mathbf{R} \mathcal{H o m}(-, \mathcal{G}): \mathbf{D}^{b}(\mathrm{Coh} / X)^{\mathrm{op}} \longrightarrow \mathbf{D}^{b}(\mathrm{Coh} / X)
$$

is its own left adjoint, and the unit and counit of adjunction are the restrictions to $\mathbf{D}^{b}(\mathrm{Coh} / X)$ of the unit and counit of adjunction on $\mathbf{D}(\mathrm{Qcoh} / X)$.

Now general category theory kicks in and tells us that, if $G$ is a functor with a left adjoint $F$, then $G$ will be an equivalence if and only if both the unit and the counit of adjunction are isomorphisms. In our particular case, where the unit and counit happen to agree, this comes down to the following.

Lemma 3.5. Choose an object $\mathcal{J} \in \mathbf{D}^{b}(\mathrm{Coh} / X)$. The object $\mathcal{J}$ is a dualizing complex if and only if, for every object $\mathcal{E} \in \mathbf{D}^{b}(\mathrm{Coh} / X)$, we have

(i) $\mathbf{R} \mathcal{H} \operatorname{Com}(\mathcal{E}, \mathcal{J}) \in \mathbf{D}^{b}(\operatorname{Coh} / X)$.

(ii) The map $\mathcal{E} \longrightarrow \mathbf{R} \mathcal{H o m}(\mathbf{R} \mathcal{H} \operatorname{lom}(\mathcal{E}, \mathcal{J})$, J $)$ is an isomorphism.

Proof. (i) is equivalent to $\mathbf{R} \mathcal{H} \operatorname{Com}(-, \mathcal{J})$ restricting to a functor from $\mathbf{D}^{b}(\mathrm{Coh} / X)$ to itself, while (ii) is equivalent to the unit and counit of the adjunction of Remark 3.4 being isomorphisms.

Slightly less trivial is the formulation

Proposition 3.6. Let $\mathcal{J}$ be an object of $\mathbf{D}^{b}(\mathrm{Coh} / X)$. Then $\mathcal{J}$ is a dualizing complex if and only if

(i) For every object $\mathcal{E} \in \mathbf{D}^{b}(\mathrm{Coh} / X)$ we have $\mathbf{R} \mathcal{H o m}(\mathcal{E}, \mathcal{J}) \in \mathbf{D}^{b}(\mathrm{Coh} / X)$.

(ii) The natural map $\mathcal{O}_{X} \longrightarrow \mathbf{R} \mathcal{H} \operatorname{Com}(\mathcal{J}, \mathcal{J})$ is an isomorphism. 
Proof. To deduce Proposition 3.6 from Lemma 3.5, we need to show that it suffices to check that the morphism

$$
\mathcal{E} \longrightarrow \mathbf{R} \mathcal{H o m}(\mathbf{R} \mathcal{H o m}(\mathcal{E}, \mathcal{J}), \mathcal{J})
$$

is an isomorphism in the special case where $\mathcal{E}=\mathcal{O}_{X}$. The useful Lemma 2.9 allows us to identify, for any $\mathcal{E}$,

$$
\mathcal{E}^{\mathbf{L}} \otimes \mathbf{R} \mathcal{H o m}(\mathcal{J}, \mathcal{J}) \cong \mathbf{R} \mathcal{H} \operatorname{Hom}(\mathbf{R} \mathcal{H o m}(\mathcal{E}, \mathcal{J}), \mathcal{J}) .
$$

The morphism we need to show an isomorphism becomes

$$
\mathcal{E} \longrightarrow \mathcal{E}^{\mathbf{L}} \otimes \mathbf{R} \mathcal{H} \text { om }(\mathcal{J}, \mathcal{J}),
$$

and now it clearly suffices to treat the case $\mathcal{E}=\mathcal{O}_{X}$.

Notation 3.7. If $X$ is a noetherian scheme and $\mathcal{J}$ is a dualizing complex on $X$, we will sometimes abbreviate the functor $\mathbf{R H o m}(-, \mathcal{J})$ to just $D_{X, \mathcal{J}}(-)$. If either $X$ or $\mathcal{J}$ is understood from the context we will feel free to drop it from the notation; thus the symbols $D_{X}(-), D_{\mathcal{J}}(-)$ and $D_{X, \mathcal{J}}(-)$ should be viewed as synonymous, where the third is the most explicit. What we constructed above, using the unit of adjunction, is a natural ismorphism $\mathcal{E} \longrightarrow D_{X, \mathcal{J}}\left(D_{X, \mathcal{J}}(\mathcal{E})\right)$.

Remark 3.8. The next obvious question is what happens if we are given, on the same scheme $X$, two dualizing complexes $\mathcal{J}$ and $\mathcal{J}$. How do they compare?

It is clear that, if we are given a dualizing complex $\mathcal{J}$, an integer $n$ and a line bundle $\mathcal{L}$, then $\Sigma^{n} \mathcal{L} \mathbf{L} \otimes \mathcal{J}$ is also a dualizing complex; tensoring with a line bundle and suspending is harmless. It is also harmless to suspend by different integers on different connected components of $X$. The remarkable fact is that this is all the freedom we have. Up to these basic moves, the dualizing complex is unique. We state this as a lemma and give the proof.

Lemma 3.9. Let $\mathcal{J}$ and $\mathcal{J}$ be two dualizing complexes on the same scheme $X$. Then $\mathcal{J}=\mathcal{L} \mathbf{L} \otimes \mathcal{J}$, where $\mathcal{L}$ is locally some suspension of a line bundle. On each connected component $X_{i} \subset X$ there is an integer $n_{i}$, a line bundle $\mathcal{L}_{i}$, and an isomorphism $\left.\mathcal{L}\right|_{X_{i}} \cong \Sigma^{n_{i}} \mathcal{L}_{i}$.

Proof. Let us define $\mathcal{L}=\mathbf{R} \mathcal{H}$ om $(\mathcal{J}, \mathcal{J})$. Now note that

$$
\begin{aligned}
\mathcal{J} & =\mathbf{R} \mathcal{H o m}\left(\mathcal{O}_{X}, \mathcal{J}\right) & & \\
& =\mathbf{R} \mathcal{H o m}(\mathbf{R} \mathcal{H o m}(\mathcal{J}, \mathcal{J}), \mathcal{J}) & & \text { by Proposition 3.6(ii) } \\
& =\mathcal{J} \mathbf{L} \otimes \mathbf{R} \mathcal{H o m}(\mathcal{J}, \mathcal{J}) & & \text { by Lemma } 2.10 \\
& =\mathcal{J} \mathbf{L}_{\otimes} \mathcal{L} & & \text { by definition of } \mathcal{L} .
\end{aligned}
$$


It remains to analyse $\mathcal{L}$ and prove that, on each connected component of $X$, it is isomorphic to the suspension of a line bundle.

Consider the functor $D_{\mathfrak{J}} D_{\mathfrak{\jmath}}$. This functor takes the complex $\mathcal{E}$ to the complex

$$
D_{\mathcal{J}}\left(D_{\mathcal{J}}(\mathcal{E})\right)=\mathbf{R} \mathcal{H} \operatorname{lom}(\mathbf{R} \mathcal{H} \operatorname{com}(\mathcal{E}, \mathcal{J}), \mathcal{J})
$$

and Lemma 2.10 identifies this with $\mathcal{E}^{\mathbf{L}} \otimes \mathbf{R} \mathcal{H} \operatorname{om}(\mathcal{J}, \mathcal{J})$. Interchanging the roles of $\mathcal{J}$ and $\mathcal{J}$, we have that $D_{\mathcal{J}}\left(D_{\mathcal{J}}(\mathcal{E})\right)$ is naturally isomorphic to $\mathcal{E}^{\mathbf{L}} \otimes \mathbf{R} \mathcal{H o m}(\mathcal{J}, \mathcal{J})$. Now the functor $D_{\mathcal{J}} D_{\mathcal{\jmath}} D_{\mathcal{\jmath}} D_{\mathcal{J}}$ is naturally isomorphic to the identity; if the units=counits of the adjunctions of Remark 3.4 are written $\eta_{i}: 1 \Longrightarrow D_{\mathcal{J}} D_{\mathcal{J}}$ and $\eta_{j}: 1 \Longrightarrow D_{\mathfrak{\jmath}} D_{\mathfrak{\jmath}}$, then the composite

$$
1 \stackrel{\eta_{i}}{\longrightarrow} D_{\mathcal{J}} D_{\mathcal{J}} \stackrel{D_{\mathcal{J}} \eta_{j} D_{\mathcal{J}}}{\longrightarrow} D_{\mathcal{J}} D_{\mathfrak{J}} D_{\mathcal{J}} D_{\mathcal{J}}
$$

provides the isomorphism. On the object $\mathcal{E} \in \mathbf{D}^{b}(\mathrm{Coh} / X)$ this isomorphism is the natural map

$$
\mathcal{E} \longrightarrow \mathcal{E}^{\mathbf{L}} \otimes \mathbf{R} \mathcal{H} \text { om }(\mathcal{J}, \mathcal{J}) \longrightarrow \mathcal{E}^{\mathbf{L}} \otimes \mathbf{R} \mathcal{H} \operatorname{lom}(\mathcal{J}, \mathcal{J}) \mathbf{L}_{\otimes} \mathbf{R} \mathcal{H o m}(\mathcal{J}, \mathcal{J}) \text {. }
$$

Applying this to $\mathcal{E}=\mathcal{O}_{X}$, we see that

$$
\mathbf{R} \mathcal{H o m}(\mathcal{J}, \mathcal{J})^{\mathbf{L}} \otimes \mathbf{R} \mathcal{H} \operatorname{lom}(\mathcal{J}, \mathcal{J}) \cong \mathcal{O}_{X} .
$$

We already defined $\mathcal{L}=\mathbf{R} \mathcal{H} \operatorname{lom}(\mathcal{J}, \mathcal{J})$; now set $\mathcal{L}^{\prime}=\mathbf{R} \mathcal{H} \circ \mathrm{m}(\mathcal{J}, \mathcal{J})$, and we deduce an isomorphism $\mathcal{L}^{\mathbf{L}} \otimes \mathcal{L}^{\prime} \cong \mathcal{O}_{X}$. We furthermore know that $\mathcal{L}$ and $\mathcal{L}^{\prime}$ are both objects in $\mathbf{D}^{b}(\mathrm{Coh} / X)$.

Now restrict everything to the stalk at a point $x \in X$. Then the stalk at $x$ of the sheaf $\mathcal{O}_{X}$ is a local ring $R$ with maximal ideal $\mathfrak{m}$. Let us write $\mathcal{L}_{x}, \mathcal{L}_{x}^{\prime}$ for the stalks at $x$ of $\mathcal{L}, \mathcal{L}^{\prime}$. Then $\mathcal{L}_{x}, \mathcal{L}_{x}^{\prime}$ are objects in $\mathbf{D}^{b}(R-$ mod $)$ satisfying $\mathcal{L}_{x} \mathbf{L}_{\otimes} \mathcal{L}_{x}^{\prime} \cong R$. Let $k=k(x)$ be the residue field of $R$. We know that $k^{\mathbf{L}} \otimes \mathcal{L}_{x}$ is a complex of $k$-vector spaces; that is it must be a direct sum of suspensions of $k$. Now consider the isomorphisms

$$
\left(k^{\mathbf{L}} \otimes \mathcal{L}_{x}\right)^{\mathbf{L}_{\otimes}} \mathcal{L}_{x}^{\prime}=k^{\mathbf{L}} \otimes\left(\mathcal{L}_{x} \mathbf{L}_{\otimes} \mathcal{L}_{x}^{\prime}\right)=k^{\mathbf{L}} \otimes R=k .
$$

We learn first that $k^{\mathbf{L}} \otimes \mathcal{L}_{x}$ must be nonzero, and by symmetry $k^{\mathbf{L}} \otimes \mathcal{L}_{x}^{\prime}$ is also nonzero. But also $k=\left(k^{\mathbf{L}} \otimes \mathcal{L}_{x}\right) \mathbf{L} \otimes \mathcal{L}_{x}^{\prime}$ is indecomposable. It follows that $k^{\mathbf{L}} \otimes \mathcal{L}_{x}$ cannot be a direct sum of more than one factor, since then $\left(k^{\mathbf{L}} \otimes \mathcal{L}_{x}\right)^{\mathbf{L}} \otimes \mathcal{L}_{x}^{\prime}$ would decompose into more than one direct summand, and each of the summands would be isomorphic to some suspension of the nontrivial $k^{\mathbf{L}} \otimes \mathcal{L}_{x}^{\prime}$. We conclude that $k^{\mathbf{L}} \otimes \mathcal{L}_{x}$ must be of the form $\Sigma^{n} k$, for some integer $n$.

Now $\mathcal{L}_{x}$ is an object in $\mathbf{D}^{b}(R-\bmod )$, and it therefore has a minimal projective resolution. We remind the reader: a minimal projective resolution is a bounded above chain complex of finitely generated, free $R$-modules, so that all the differentials vanish modulo $\mathfrak{m}$. The object $k^{\mathbf{L}} \otimes \mathcal{L}_{x}$ can be 
computed by taking the ordinary tensor product of $k$ with the minimal projective resolution. The fact that $k^{\mathbf{L}} \otimes \mathcal{L}_{x} \cong \Sigma^{n} k$ tells us that the minimal projective resolution must be very sparse; it consists of a single free module, of rank 1 , concentrated in degree $n$. That is $\mathcal{L}_{x} \cong \Sigma^{n} R$.

So far we have focused on the stalk of a single point $x \in X$. We have a chain complex $\mathcal{L} \in \mathbf{D}^{b}(\mathrm{Coh} / X)$, that is a bounded chain complex of coherent sheaves on $X$. We have shown that, at the point $x$, the stalks of the cohomology sheaves $\mathbb{H}^{k}(\mathcal{L})$ vanish if $k \neq-n$, and if $k=-n$ we obtained an isomorphism of the stalk at $x$ of $\mathbb{H}^{-n}(\mathcal{L})$ with the free module $R$ of rank 1. But the sheaves $\mathbb{H}^{k}(\mathcal{L})$ are coherent sheaves on $X$, all but finitely many of which vanish. It follows that there exists a Zariski open set $U \subset X$ so that, on $U$, the coherent sheaves $\mathbb{H}^{k}(\mathcal{L})$ satisfy

$$
\left.\mathbb{H}^{k}(\mathcal{L})\right|_{U}= \begin{cases}0 & \text { if } k \neq-n \\ \overline{\mathcal{L}} & \text { if } k=-n\end{cases}
$$

where $\overline{\mathcal{L}}$ is some line bundle on $U$. Now the integer $n$ is locally constant, and must be constant on connected components. On each connected component $X_{i}$ there is an integer $n_{i}$ so that $\mathbb{H}^{k}(\mathcal{L})=0$ for $k \neq-n_{i}$, and $\mathbb{H}^{-n_{i}}(\mathcal{L})$ is a line bundle. This makes $\left.\mathcal{L}\right|_{X_{i}}$ a complex whose cohomology sheaves vanish except in only one dimension, and hence it must be isomorphic in $\mathbf{D}^{b}\left(\mathrm{Coh} / X_{i}\right)$ to $\Sigma^{n_{i}} \mathbb{H}^{-n_{i}}(\mathcal{L})$.

Remark 3.10. Let $\mathcal{J}$ be a dualizing complex. It is an object of $\mathbf{D}^{b}(\mathrm{Coh} / X)$, and has a bounded-below injective resolution. In the homotopy category $\mathbf{K}(\operatorname{Inj} / X)$, whose objects are chain complexes of injective quasicoherent sheaves on $X$, there is a bounded-below complex $I$ and a quasi-isomorphism $\mathcal{J} \longrightarrow I$. Furthermore, $I$ is unique up to homotopy. If $\mathcal{J}$ is another dualising complex, Lemma 3.9 informs us that $\mathcal{J}=\mathcal{L}^{\mathbf{L}} \otimes \mathcal{J}$, with $\mathcal{L}$ locally isomorphic to a shift of a line bundle. Obviously, $\mathcal{L} \otimes I$ is an injective resolution for $\mathcal{J}$.

While the injective resolutions $I \in \mathbf{K}(\operatorname{Inj} / X)$ of dualizing complexes $\mathcal{J}$ are only determined up to twisting by complexes $\mathcal{L}$, the Hom-complexes $\mathcal{H} \operatorname{com}(I, I)$ are objects of $\mathbf{K}($ Flat $/ X)$ well defined up to homotopy; we have

$$
\mathcal{H o m}(I, I) \cong \mathcal{H} \operatorname{Hom}(\mathcal{L} \otimes I, \mathcal{L} \otimes I) .
$$

Even though the dualizing complex $\mathcal{J}$ started its life as an object of the derived category $\mathbf{D}^{b}(\mathrm{Coh} / X)$, subject to conditions that appear innocuous enough, we have learned

(i) The object $\mathcal{J}$ is unique up to tensor by an $\mathcal{L}$, with $\mathcal{L}$ locally isomorphic to a shift of a line bundle.

(ii) If we replace $\mathcal{J}$ by its injective resolution, then the object $\mathcal{H} \operatorname{Hom}(\mathcal{J}, \mathcal{J})$ is a well-defined complex of flat $\mathcal{O}_{X}$-modules, unique up to homotopy. 
In Proposition 3.6 we learned that the natural map $\mathcal{O}_{X} \longrightarrow \mathcal{H}$ om $(\mathcal{J}, \mathcal{J})$ must be an isomorphism in $\mathbf{D}^{b}(\mathrm{Coh} / X)$. It is a homology isomorphism of complexes of flat modules, but not usually a homotopy equivalence.

Remark 3.11. Next we will study what happens to dualizing complexes under a morphism of schemes $f: X \longrightarrow Y$. More precisely, whenever we have a pair of noetherian, separated schemes $X$ and $Y$, there is a pair of derived categories $\mathbf{D}(\mathrm{Qcoh} / X)$ and $\mathbf{D}(\mathrm{Qcoh} / Y)$. A morphism of schemes $f: X \longrightarrow Y$ induces several morphisms between these derived categories. There is the pushforward functor $\mathbf{R} f_{*}: \mathbf{D}(\mathrm{Qcoh} / X) \longrightarrow \mathbf{D}(\mathrm{Q} \operatorname{coh} / Y)$, which has a left adjoint $\mathbf{L} f^{*}$ and a right adjoint $f^{!}$. Assuming that either $\mathbf{D}^{b}(\mathrm{Coh} / X)$ or $\mathbf{D}^{b}(\mathrm{Coh} / Y)$ has a dualizing complex, one can ponder whether some of the three functors above might respect it. There are basically two theorems.

Theorem 3.12. Let $f: X \longrightarrow Y$ be an open immersion, and assume that $\mathcal{J}$ is a dualizing complex on $Y$. Then $\mathbf{L} f^{*} \mathcal{J}$ is a dualizing complex on $X$.

Proof. First of all a point of notation: since $f$ is an open immersion the functor $f^{*}$ is exact, and hence is equal to its derived functor. We will write $f^{*}$ for $\mathbf{L} f^{*}$. Also, the fact that $\mathcal{J} \in \mathbf{D}^{b}(\mathrm{Coh} / Y)$ clearly implies that $\mathbf{L} f^{*} \mathcal{J}=f^{*} \mathcal{J}$ belongs to $\mathbf{D}^{b}(\mathrm{Coh} / X)$.

By Proposition 3.6 it suffices to prove two things:

(i) For all objects $\mathcal{E} \in \mathbf{D}^{b}(\mathrm{Coh} / X)$ we will show that $\mathbf{R} \mathcal{H}$ om $\left(\mathcal{E}, f^{*} \mathcal{J}\right)$ belongs to $\mathbf{D}^{b}(\mathrm{Coh} / X)$.

(ii) We will show that the natural map $\mathcal{O}_{X} \longrightarrow \mathbf{R} \mathcal{H o m}\left(f^{*} \mathcal{J}, f^{*} \mathcal{J}\right)$ is an isomorphism.

Let us begin with (i). Since coherent sheaves and morphisms of coherent sheaves can be extended from the open set $X$ to all of $Y$, any object $\mathcal{E} \in$ $\mathbf{D}^{b}(\mathrm{Coh} / X)$ is isomorphic to $f^{*} \mathcal{G}$, where $\mathcal{G} \in \mathbf{D}^{b}(\mathrm{Coh} / Y)$. Therefore

$$
\begin{aligned}
\mathbf{R} \mathcal{H o m}\left(\mathcal{E}, f^{*} \mathcal{J}\right) & =\mathbf{R} \mathcal{H o m}\left(f^{*} \mathcal{G}, f^{*} \mathcal{J}\right) \\
& =f^{*} \mathbf{R} \mathcal{H o m}(\mathcal{G}, \mathcal{J}) \quad \text { by Lemma } 2.4 .
\end{aligned}
$$

Now $\mathbf{R} \mathcal{H} \operatorname{Com}(\mathcal{G}, \mathcal{J})$ is in $\mathbf{D}^{b}(\mathrm{Coh} / Y)$ because $\mathcal{J}$ is a dualizing complex on $Y$, and $f^{*}$ takes $\mathbf{D}^{b}(\mathrm{Coh} / Y)$ to $\mathbf{D}^{b}(\mathrm{Coh} / X)$.

Next we prove (ii). Lemma 2.4 informs us that $\mathbf{R} \mathcal{H}$ om $\left(f^{*} \mathcal{J}, f^{*} \mathcal{J}\right)=$ $f^{*} \mathbf{R} \mathcal{H o m}(\mathcal{J}, \mathcal{J})$, and the fact that $\mathcal{J}$ is a dualizing complex on $Y$ says that $\mathbf{R} \mathcal{H o m}(\mathcal{J}, \mathcal{J})=\mathcal{O}_{Y}$. We deduce that $\mathbf{R} \mathcal{H} \operatorname{lom}\left(f^{*} \mathcal{J}, f^{*} \mathcal{J}\right)=f^{*} \mathcal{O}_{Y}=\mathcal{O}_{X}$. We leave it to the reader to check that the isomorphism we have produced $\mathcal{O}_{X} \cong \mathbf{R} \mathcal{H o m}\left(f^{*} \mathcal{J}, f^{*} \mathcal{J}\right)$ is the natural map.

Remark 3.13. The next theorem gives sufficient conditions for $f^{!}$to take a dualizing complex on $Y$ to a dualizing complex on $X$. Before we state the 
theorem, it might help if we remind the reader of what boundedness properties are always, unconditionally true, for any morphism $f: X \longrightarrow Y$; this might help separate what is formal from the genuinely restrictive hypotheses of the theorem.

We are assuming that $X$ and $Y$ are noetherian and separated. This means that the functor $\mathbf{R} f_{*}$ can be computed using Cech cohomology with respect to an affine open cover of $X$, and this cover may be taken finite. If $\mathcal{E} \in \mathbf{D}(\mathrm{Q} \operatorname{coh} / X)$ is bounded it follows that $\mathbf{R} f_{*} \mathcal{E}$ will also be a bounded complex. That much is free. But we have no obvious way to control the size (as in coherence versus quasicoherence) of the finitely many cohomology sheaves of $\mathbf{R} f_{*} \mathcal{E}$.

It turns out to be a strong restriction on $f$ to assume that

$$
\mathbf{R} f_{*}: \mathbf{D}(\mathrm{Q} \operatorname{coh} / X) \longrightarrow \mathbf{D}(\mathrm{Q} \operatorname{coh} / Y)
$$

respects the bounded, coherent subcategories. In symbols: it may happen that $\mathbf{R} f_{*}$ will take the subcategory $\mathbf{D}^{b}(\mathrm{Coh} / X) \subset \mathbf{D}(\mathrm{Q} \operatorname{coh} / X)$ to the subcategory $\mathbf{D}^{b}(\mathrm{Coh} / Y) \subset \mathbf{D}(\mathrm{Q} c o h / Y)$, but it certainly does not come for free. The usual sufficient condition is that the map $f: X \longrightarrow Y$ be a proper morphism of finite type. This concludes what we will need to know concerning the functor $\mathbf{R} f_{*}$, to make sense of the statement of Theorem 3.14; the preservation of boundedness is formal, the preservation of coherence is not.

Next we want to look at the properties of the functor $f^{!}$which play a role in the statement of Theorem 3.14. It helps to begin by returning briefly to the functor $\mathbf{R} f_{*}$, and observing a little more closely the precise bounds that the Cech complex gives. Suppose that $X$ admits a cover by $n+1$ affine open sets; then the Cech complex computing $\mathbf{R} f_{*}$ has length $n$. If $\mathcal{E}$ is an object in $\mathbf{D}^{<m}(\mathrm{Q} \operatorname{coh} / X)$, that is $\mathcal{E} \in \mathbf{D}(\mathrm{Qcoh} / X)$ is quasiisomorphic to a complex vanishing in degrees $\geq m$, then $\mathbf{R} f_{*} \mathcal{E}$ must be in $\mathbf{D}^{<m+n}(\mathrm{Qcoh} / Y)$; a Cech complex of length $n$ can only raise the cohomological degree by at most $n$. This means that for any $\mathcal{F} \in \mathbf{D}^{\geq m+n}(\mathrm{Q} c o h / Y)$ we have $\operatorname{Hom}\left(\mathbf{R} f_{*} \mathcal{E}, \mathcal{F}\right)=0$. Adjunction gives us $\operatorname{Hom}\left(\mathcal{E}, f^{!} \mathcal{F}\right)=0$, and this is true for all $\mathcal{E} \in \mathbf{D}^{<m}(\mathrm{Qcoh} / X)$. Hence $f^{!} \mathcal{F} \in \mathbf{D}^{\geq m}(\mathrm{Q} c o h / X)$. We have just shown that the functor $f^{!}$must take bounded-below complexes to bounded-below complexes. In particular the image under $f^{!}$of the subcategory $\mathbf{D}^{b}(\mathrm{Coh} / Y) \subset \mathbf{D}(\mathrm{Q} c o h / Y)$ must lie in $\mathbf{D}^{+}(\mathrm{Q} c o h / X) \subset \mathbf{D}(\mathrm{Q} c o h / X)$.

That ends our free ride. There is no formal reason to expect the functor $f^{!}$to take $\mathbf{D}^{b}(\mathrm{Coh} / Y) \subset \mathbf{D}(\mathrm{Q} \operatorname{coh} / Y)$ into $\mathbf{D}^{b}(\mathrm{Coh} / X)$, or even into the larger $\mathbf{D}_{\mathrm{Coh}}^{+}(\mathrm{Q} \operatorname{coh} / X)$. We remind the reader: the objects in the category $\mathbf{D}_{\mathrm{Coh}}^{+}(\mathrm{Q} \operatorname{coh} / X)$ are the bounded-below chain complexes of quasicoherent sheaves, whose cohomology is all coherent. 
Next we prove:

Theorem 3.14. Let $f: X \longrightarrow Y$ be a morphism of noetherian, separated schemes so that

(i) The functor $\mathbf{R} f_{*}: \mathbf{D}(\mathrm{Qcoh} / X) \longrightarrow \mathbf{D}(\mathrm{Qcoh} / Y)$ takes $\mathbf{D}^{b}(\mathrm{Coh} / X) \subset$ $\mathbf{D}(\mathrm{Qcoh} / X)$ into $\mathbf{D}^{b}(\mathrm{Coh} / Y) \subset \mathbf{D}(\mathrm{Q} \operatorname{coh} / Y)$.

(ii) The functor $f^{!}: \mathbf{D}(\mathrm{Qcoh} / Y) \longrightarrow \mathbf{D}(\mathrm{Qcoh} / X)$ takes $\mathbf{D}^{b}(\mathrm{Coh} / Y) \subset$ $\mathbf{D}(\mathrm{Q} c o h / Y)$ into $\mathbf{D}_{\mathrm{Coh}}^{+}(\mathrm{Q} \operatorname{coh} / X) \subset \mathbf{D}(\mathrm{Q} \operatorname{coh} / X)$.

If $\mathcal{J}$ is a dualizing complex on $Y$ then $f^{!} \mathcal{J}$ is a dualizing complex on $X$.

Remark 3.15. The reader might wish to compare Theorem 3.14 with older results in the literature; see, for example, [15, Remark 2, p. 299], [66, Corollary 3, p. 396], [2, Proposition 2.5.11] and [45, §9.1 and §9.2].

Reminder 3.16. In the proof of Theorem 3.14 we will appeal to the technical result [46, Theorem 4.2]; let us therefore remind the reader. The technical result asserts the following: in the category $\mathbf{D}(\mathrm{Qcoh} / X)$ there is a compact generator $S$ which detects non-vanishing high cohomology. Precisely, this means that there is an integer $A=A(S)$, depending only on $S$, so that

(iii) If $Y$ is an object of $\mathbf{D}(\mathrm{Qcoh} / X)$, with $\operatorname{Hom}\left(S, \Sigma^{k} Y\right)=0$ for all $k \geq n$, then $\mathbb{H}^{k}(Y)=0$ for all $k \geq n+A$.

Perhaps we should explain the notation: the $\mathbb{H}^{k}$ means the $k$ th cohomology sheaf of the chain complex of sheaves. The result informs us that we can tell whether the sheaf cohomology vanishes, above a certain degree, just by computing some groups, namely $\operatorname{Hom}\left(S, \Sigma^{k} Y\right)$.

Proof. We now prove Theorem 3.14. As in the proof of Theorem 3.12 we appeal to Proposition 3.6. It suffices to establish two things:

(iv) For all objects $\mathcal{G} \in \mathbf{D}^{b}(\mathrm{Coh} / X)$ we will show that $\mathbf{R} \mathcal{H}$ om $\left(\mathcal{G}, f^{!} \mathcal{J}\right)$ belongs to $\mathbf{D}^{b}(\mathrm{Coh} / X)$. The case where $\mathcal{G}=\mathcal{O}_{X}$ will prove that $f^{!} \mathcal{J} \in \mathbf{D}^{b}(\mathrm{Coh} / X)$.

(v) We will show that the natural map $\mathcal{O}_{X} \longrightarrow \mathbf{R} \mathcal{H}$ om $\left(f^{!} \mathcal{J}, f^{!} \mathcal{J}\right)$ is an isomorphism.

Let us begin with (iv). What we are given is that $\mathcal{G}$ belongs to $\mathbf{D}^{b}(\mathrm{Coh} / X)$, and hypothesis (ii) of the Theorem says that $f^{!} \mathcal{J}$ must belong to $\mathbf{D}_{\text {Coh }}^{+}(\mathrm{Qcoh} / X)$. It follows that $\mathbf{R} \mathcal{H o m}\left(\mathcal{G}, f^{!} \mathcal{J}\right)$ has to be in $\mathbf{D}_{\text {Coh }}^{+}(\mathrm{Qcoh} / X)$. We remind the reader: the complex $\mathcal{G}$ is finite, and hence we can immediately reduce to the case where $\mathcal{G}$ is a single coherent sheaf concentrated in degree 0 . If $\mathcal{G}$ is a single sheaf, then there is a spectral sequence converging to the cohomology sheaves of the complex $\mathbf{R} \mathcal{H} \operatorname{lom}\left(\mathcal{G}, f^{!} \mathcal{J}\right)$, whose $E_{2}^{i j}$ term is $\mathcal{E} x t^{i}\left(\mathcal{G}, \mathbb{H}^{j}\left(f^{!} \mathcal{J}\right)\right)$. The sheaves $E_{2}^{i j}$ are all coherent, and $\mathbb{H}^{i+j} \mathbf{R} \mathcal{H}$ om $\left(\mathcal{G}, f^{!} \mathcal{J}\right)$ is a finite extension of subquotients of them, hence also coherent. 
So much for coherence; to establish (iv) it remains to show that the cohomology of $\mathbf{R} \mathcal{H} \operatorname{lom}\left(\mathcal{G}, f^{!} \mathcal{J}\right)$ is bounded above. For this we use [46, Theorem 4.2]. Choose an object $S$ as in ijDEFANGED.778 Reminder 3.16. We know that the vanishing, for sufficiently large $k$, of the two sequences

$$
\mathbb{H}^{k}\left(\mathbf{R} \mathcal{H o m}\left(\mathcal{G}, f^{!} \mathcal{J}\right)\right), \quad \operatorname{Hom}\left(S, \Sigma^{k} \mathbf{R} \mathcal{H} \operatorname{Hom}\left(\mathcal{G}, f^{!} \mathcal{J}\right)\right)
$$

is equivalent. And the point is that we can compute the groups on the right; they are

$$
\begin{aligned}
& \operatorname{Hom}\left(S, \mathbf{R} \mathcal{H o m}\left(\mathcal{G}, \Sigma^{k} f^{!} \mathcal{J}\right)\right)=\operatorname{Hom}\left(S^{\mathbf{L}} \otimes \mathcal{G}, \Sigma^{k} f^{!} \mathcal{J}\right) \\
& =\operatorname{Hom}\left(\mathbf{R} f_{*}\left(S^{\mathbf{L}} \otimes \mathcal{G}\right), \Sigma^{k} \mathcal{J}\right) .
\end{aligned}
$$

Now $S$ is a perfect complex; locally it is a bounded complex of finitely generated projectives. The complex $\mathcal{G}$ is locally a bounded complex of finite modules, and the derived tensor product is locally just the tensor product; locally $S^{\mathbf{L}} \otimes \mathcal{G}$ is a bounded complex of finite modules. Thus $S^{\mathbf{L}} \otimes \mathcal{G}$ belongs to $\mathbf{D}^{b}(\mathrm{Coh} / X)$, and by (i) it follows that $\mathbf{R} f_{*}\left(S^{\mathbf{L}} \otimes \mathcal{G}\right)$ must belong to $\mathbf{D}^{b}(\mathrm{Coh} / Y)$. The complex $\mathcal{J}$ is by hypothesis a dualizing complex, and hence $\mathbf{R} \mathcal{H o m}\left(\mathbf{R} f_{*}\left(S^{\mathbf{L}} \otimes \mathcal{G}\right)\right.$, J $)$ must also belong to $\mathbf{D}^{b}(\mathrm{Coh} / Y)$. Its cohomology may be computed using the Cech complex on $Y$, and hence is bounded. Therefore

$$
\operatorname{Hom}\left(\mathbf{R} f_{*}\left(S^{\mathbf{L}} \otimes \mathcal{G}\right), \Sigma^{k} \mathfrak{J}\right)=H^{k} \mathbf{R} \mathcal{H o m}\left(\mathbf{R} f_{*}\left(S^{\mathbf{L}} \otimes \mathcal{G}\right), \mathcal{J}\right)
$$

must vanish for $k$ sufficiently large.

It remains to prove $(\mathrm{v})$; we must show that the natural map $\rho: \mathcal{O}_{X} \longrightarrow$ $\mathbf{R} \mathcal{H o m}\left(f^{!} \mathcal{J}, f^{!} \mathcal{J}\right)$ is an isomorphism. We have already proved, in (iv), that for any $\mathcal{G} \in \mathbf{D}^{b}(\operatorname{Coh} / X)$ we have $\mathbf{R} \mathcal{H} \operatorname{Com}\left(\mathcal{G}, f^{!} \mathcal{J}\right) \in \mathbf{D}^{b}(\operatorname{Coh} / X)$. If we put $\mathcal{G}=\mathcal{O}_{X}$ we conclude that $f^{!} \mathcal{J} \in \mathbf{D}^{b}(\mathrm{Coh} / X)$, and then if we put $\mathcal{G}=f^{!} \mathcal{J}$ we deduce that $\mathbf{R} \mathcal{H} \operatorname{lom}\left(f^{!} \mathcal{J}, f^{!} \mathcal{J}\right)$ must also be in $\mathbf{D}^{b}(\mathrm{Coh} / X)$. The morphism $\rho: \mathcal{O}_{X} \longrightarrow \mathbf{R} \mathcal{H}$ om $\left(f^{!} \mathcal{J}, f^{!} \mathcal{J}\right)$ is therefore a map in $\mathbf{D}^{b}(\mathrm{Coh} / X)$, and the mapping cone lies in $\mathbf{D}^{b}(\mathrm{Coh} / X)$. Locally the mapping cone has a minimal resolution; it will vanish if and only if, for all closed points $x \in X$, the tensor product with $k(x)$ vanishes. To prove that $\rho$ an isomorphism it therefore suffices to check that $k(x)^{\mathbf{L}} \otimes \rho$ is an isomorphism for every closed point $x \in X$. That is, we wish to study the natural map

$$
k(x) \longrightarrow k(x)^{\mathbf{L}} \otimes \mathbf{R} \mathcal{H} \text { om }\left(f^{\prime} \mathfrak{J}, f^{!} \mathcal{J}\right)
$$

and prove it an isomorphism.

Lemma 2.10 helps. The point $x$ is assumed closed, guaranteeing that the skyscraper sheaf $k(x)$, concentrated at the point $x \in X$, is a coherent sheaf. Lemma 2.10 therefore gives us a natural isomorphism

$$
k(x)^{\mathbf{L}} \otimes \mathbf{R} \mathcal{H} \text { om }\left(f^{\prime} \mathcal{J}, f^{!} \mathcal{J}\right) \longrightarrow \mathbf{R} \mathcal{H} \text { om }\left(\mathbf{R} \mathcal{H} \operatorname{Hom}\left(k(x), f^{!} \mathcal{J}\right), f^{\prime} \mathcal{J}\right)
$$


and we are reduced to proving that the natural map

$$
k(x) \longrightarrow \mathbf{R} \mathcal{H} \text { om }\left(\mathbf{R} \mathcal{H} \operatorname{lom}\left(k(x), f^{\prime} \mathcal{J}\right), f^{!} \mathcal{J}\right),
$$

is an isomorphism. In the case of skyscraper sheaves concentrated at a single point we can safely apply $\mathbf{R} f_{*}$, and then check that the induced map on $Y$ is an isomorphism; after all the only effect is to push the problem forward to the point $f(x) \in Y$. But now observe

$$
\begin{aligned}
\mathbf{R} f_{*} \mathbf{R} \mathcal{H} \text { om }(\mathbf{R} \mathcal{H o m}(k(x), & \left.\left.f^{!} \mathcal{J}\right), f^{!} \mathcal{J}\right) \cong \\
& \cong \mathbf{R} \mathcal{H} \operatorname{lom}\left(\mathbf{R} f_{*} \mathbf{R} \mathcal{H} \operatorname{lom}\left(k(x), f^{!} \mathcal{J}\right), \mathcal{J}\right) \\
& \cong \mathbf{R} \mathcal{H} \operatorname{lom}\left(\mathbf{R} \mathcal{H} \operatorname{lom}\left(\mathbf{R} f_{*} k(x), \mathcal{J}\right), \mathcal{J}\right) \\
& \cong \mathbf{R} f_{*} k(x) .
\end{aligned}
$$

The careful reader should note that, in the above isomorphisms, we assert that two morphisms $\mathbf{R} f_{*} \mathbf{R} \mathcal{H} \operatorname{Hom}\left(\mathcal{E}, f^{!} \mathcal{J}\right) \longrightarrow \mathbf{R} \mathcal{H}$ om $\left(\mathbf{R} f_{*} \mathcal{E}, \mathcal{J}\right)$ are isomorphisms. In both cases $\mathcal{E} \in \mathbf{D}^{b}(\mathrm{Coh} / X)$ is supported at a single point $x \in X$, meaning that both complexes we are trying to prove isomorphic are acyclic away from $f(x) \in Y$. The fact that the natural map is an isomorphism may therefore be checked after taking global sections. The reader need not worry that we might be appealing to some subtle facts about $f^{\text {! }}$ commuting with base change, and there is no need to verify the hypotheses of [51, Lemma 6.1 and Proposition 6.2].

The proof is now complete.

Remark 3.17. The hypothesis of Theorem 3.14 are that (i) $\mathbf{R} f_{*}$ take $\mathbf{D}^{b}(\mathrm{Coh} / X)$ to $\mathbf{D}^{b}(\mathrm{Coh} / Y)$, and (ii) $f^{!}$take $\mathbf{D}^{b}(\mathrm{Coh} / Y)$ to $\mathbf{D}_{\mathrm{Coh}}^{+}(\mathrm{Qcoh} / X)$. In Reminder 3.13 we noted that (i) holds provided $f$ is a proper morphism of finite type. All we observed concerning (ii) was that it is not automatic; we made no mention of any interesting examples of $f \mathrm{~s}$ which satisfy (ii). It is time to remedy this. We begin with the easy

Lemma 3.18. If $f: X \longrightarrow Y$ is a finite morphism, then $f^{!}: \mathbf{D}(\mathrm{Qcoh} / Y) \longrightarrow$ $\mathbf{D}(\mathrm{Qcoh} / X)$ takes $\mathbf{D}^{b}(\mathrm{Coh} / Y)$ to $\mathbf{D}_{\mathrm{Coh}}^{+}(\mathrm{Q} \operatorname{coh} / X)$.

Proof. If $f$ is a finite morphism then it is affine; the sheaf $f_{*} \mathcal{O}_{X}$ is a coherent sheaf of $\mathcal{O}_{Y}$-algebras on $Y$, and $X$ is simply $\operatorname{Spec}\left(\mathcal{O}_{X}\right)$. The functor $f_{*}$ is exact, and $\mathbf{R} f_{*}$ is just the forgetful functor, which takes a chain complex $C$ of $\mathcal{O}_{X}-$ modules on $X$ and views $f_{*} C$ as a chain complex of $\mathcal{O}_{Y}$-modules via the homomorphism $\mathcal{O}_{Y} \longrightarrow f_{*} \mathcal{O}_{X}$; there is no need to derive the functor $f_{*}$.

The right adjoint, or more precisely $f_{*}$ of the right adjoint, is the functor which takes a complex $D$ of $\mathcal{O}_{Y}$ modules on $Y$, and produces out of it the 
complex $\mathbf{R} \mathcal{H} m_{\mathcal{O}_{Y}}\left(f_{*} \mathcal{O}_{X}, D\right)$. Perhaps it might be clearer to restrict to an open affine of $Y$, giving the local description. Replacing $Y$ by an open affine subset and $X$ by the inverse image, we have that $Y=\operatorname{Spec}(R)$ and $X=$ $\operatorname{Spec}(S)$. The morphism $f: X \longrightarrow Y$ comes from a ring homomorphism $\varphi: R \longrightarrow S$, and the fact that $f$ is finite means that $S$ is finite as an $R_{-}$ module. The functor $f^{!}$takes a complex $D$ of $R$-modules to $\mathbf{R H o m}_{R}(S, D)$. We are assuming that $D$ is a bounded complex of finite $R$-modules, and wish to show that $f^{!} D$ is a bounded-below complex of $S$-modules, whose cohomology modules are finite over $S$.

Easy reduction tells us that we may assume the finite complex $D$ is of length 1 ; we may take $D$ to be a single, finite $R$-module concentrated in degree 0 . The cohomology of the complex $f^{!} D$ is then $\operatorname{Ext}^{i}(S, D)$; it vanishes in negative degrees, and is always finite, either as an $R$-module or as an $S$ module.

Example 3.19. Suppose $Y$ is a any (noetherian, separated) scheme. Then the sheaf $\mathcal{O}_{Y}$ certainly belongs to $\mathbf{D}^{b}(\mathrm{Coh} / Y)$, and clearly satisfies $\mathbf{R} \mathcal{H o m}\left(\mathcal{O}_{Y}, \mathcal{O}_{Y}\right)=\mathcal{O}_{Y}$. This much is free.

If we assume that $Y$ is finite dimensional and regular, or more generally that it is Gorenstein, then the sheaf $\mathcal{O}_{Y}$ has finite injective dimension and therefore, for every object $\mathcal{E} \in \mathbf{D}^{b}(\mathrm{Coh} / Y)$, we have that $\mathbf{R} \mathcal{H o m}\left(\mathcal{E}, \mathcal{O}_{Y}\right)$ has only finitely many non-vanishing cohomology sheaves. It is an easy exercise to show that they are all coherent; therefore $\mathbf{R} \mathcal{H} \operatorname{om}\left(\mathcal{E}, \mathcal{O}_{Y}\right)$ must belong to $\mathbf{D}^{b}(\mathrm{Coh} / Y)$. We conclude that $\mathcal{O}_{Y}$ is a dualizing complex for $Y$.

From Lemma 3.18 and Theorem 3.14 we know that, if $f: X \longrightarrow Y$ is any finite morphism, then $f^{!} \mathcal{O}_{Y}$ is a dualizing complex on $X$. The special case where $f: X \longrightarrow Y$ is a closed immersion tells us that any scheme, which admits a closed immersion into a Gorenstein scheme, must have a dualizing complex. Dualizing complexes are quite common.

\section{WHEN $\mathbf{R} f_{*}$ RESPECTS COMPACTS}

Let $f: X \longrightarrow Y$ be a morphism of separated, noetherian schemes. Theorem 3.14 gives us sufficient conditions for the functor $f^{!}$to take dualizing complexes on $Y$ to dualizing complexes on $X$. The conditions on $f$ come in two components: the first, part (i) of Theorem 3.14, says that the functor $\mathbf{R} f_{*}$ should take $\mathbf{D}^{b}(\mathrm{Coh} / X)$ to $\mathbf{D}^{b}(\mathrm{Coh} / Y)$. This hypothesis we understand; see Remark 3.13. Part (ii) is the restriction that $f^{!}$should take $\mathbf{D}^{b}(\mathrm{Coh} / Y)$ to $\mathbf{D}_{\mathrm{Coh}}^{+}(\mathrm{Q} \operatorname{coh} / X)$. So far, the only example we have of an $f$ satisfying (ii) comes from Lemma 3.18; the hypothesis is satisfied for any finite morphism $f$. It turns out that there is another large class of $f$ s satisfying the condition in Theorem 3.14(ii), and this section is devoted to 
studying them. We do not understand this class fully; the unsatisfactory state of our current knowledge will be made precise in Conjecture 4.16.

Consider the morphisms $f: X \longrightarrow Y$ for which $\mathbf{R} f_{*}$ takes compacts to compacts; in the literature they sometimes go by the name quasi-perfect. Suppose Conjecture 4.16 is true. Assume $f: X \longrightarrow Y$ is a quasi-perfect morphism of noetherian, separated schemes, we will show that $f^{!}$takes $\mathbf{D}^{b}(\mathrm{Coh} / Y)$ to $\mathbf{D}^{b}(\mathrm{Coh} / X)$; it most certainly satisfies the hypothesis in Theorem 3.14(ii). In other words, if the reader is willing to believe Conjecture 4.16, then Theorem 3.14 applies to quasi-perfect $f \mathrm{~s}$.

There is a refinement; Conjecture 4.16 can be made separately for each scheme $Z$. It suffices, in the paragraph above, to know that the conjecture is true for $X$. Precisely: if $f: X \longrightarrow Y$ is a quasi-perfect morphism of noetherian, separated schemes, and if Conjecture 4.16 is true for $X$, then we already know that $f^{!}$takes $\mathbf{D}^{b}(\mathrm{Coh} / Y)$ to $\mathbf{D}^{b}(\mathrm{Coh} / X)$. In this section and in $\S 5$ we will explain this, and show that there are many classes of $X \mathrm{~s}$ which satisfy Conjecture 4.16.

Quasi-perfect morphisms $f$ have been studied extensively elsewhere, and it seems remarkable that we will be able to say something new about them. Because this section might be of independent interest, to people who could not care less about dualizing complexes, we depart from our usual conventions in the article. For this section we drop the hypothesis that our schemes should be noetherian; during most of the section we will only assume them to be quasicompact and separated.

Reminder 4.1. We remind the reader: if $X$ is a quasicompact, separated scheme then the category $\mathbf{D}(\mathrm{Q} \operatorname{coh} / X)$ is compactly generated, and therefore has products; see [52, Proposition 8.4.6]. Next we note:

Lemma 4.2. Let $f: X \longrightarrow Y$ be a morphism of quasicompact, separated schemes, and assume $\mathbf{R} f_{*}: \mathbf{D}(\mathrm{Qcoh} / X) \longrightarrow \mathbf{D}(\mathrm{Qcoh} / Y)$ takes compacts to compacts. Then the left adjoint $\mathbf{L} f^{*}: \mathbf{D}(\mathrm{Qcoh} / Y) \longrightarrow \mathbf{D}(\mathrm{Qcoh} / X)$ respects products.

Proof. Let $\left\{y_{\lambda}, \lambda \in \Lambda\right\}$ be a set of objects in $\mathbf{D}($ Qcoh $/ Y)$. We wish to show that the natural map

$$
\varphi: \mathbf{L} f^{*}\left(\prod_{\lambda \in \Lambda} y_{\lambda}\right) \longrightarrow \prod_{\lambda \in \Lambda} \mathbf{L} f^{*} y_{\lambda}
$$

is an isomorphism. Since $\mathbf{D}(\mathrm{Q} \operatorname{coh} / X)$ is compactly generated it suffices to show that, for every compact object $\varepsilon \in \mathbf{D}(\mathrm{Qcoh} / X)$, the functor $\operatorname{Hom}_{X}(\mathcal{E},-)$ takes $\varphi$ to an isomorphism. Of course we can factor the functor 
$\operatorname{Hom}_{X}(\mathcal{E},-)$; it can be expressed as a composite

$\mathbf{D}(\mathrm{Q} \operatorname{coh} / X) \stackrel{\mathbf{R} \mathcal{H} o m(\varepsilon,-)}{\longrightarrow} \mathbf{D}(\mathrm{Q} \operatorname{coh} / X) \stackrel{\mathbf{R} f_{*}}{\longrightarrow} \mathbf{D}(\mathrm{Q} \operatorname{coh} / Y) \stackrel{H^{0}}{\longrightarrow} \mathcal{A} b$, and it clearly suffices to prove that the shorter composite

$$
\mathbf{D}(\mathrm{Q} \operatorname{coh} / X) \stackrel{\mathbf{R} \mathcal{H} o m(\varepsilon,-)}{\longrightarrow} \mathbf{D}(\mathrm{Q} \operatorname{coh} / X) \stackrel{\mathbf{R} f_{*}}{\longrightarrow} \mathbf{D}(\mathrm{Q} \operatorname{coh} / Y)
$$

takes $\varphi$ to an isomorphism. In Reminder 2.7 we saw that $\mathcal{E}$ is strongly dualizable and hence the functor $\mathbf{R} \mathcal{H} o m(\mathcal{E},-)$ identifies with $\mathcal{E}^{\vee} \mathbf{L}_{\otimes}-$. Parts (ii) and (iii) of Reminder 2.7 assure us that $\mathcal{E}^{\vee}$ is compact, while Reminder 2.7(i) informs us that every compact object can be written as $\mathcal{E}^{\vee}$ for some $\mathcal{E}$. We are therefore reduced to proving that, for any compact object $\varepsilon \in \mathbf{D}(\mathrm{Q} \operatorname{coh} / X)$, the composite

$$
\mathbf{D}(\mathrm{Q} \operatorname{coh} / X) \stackrel{\varepsilon^{\mathbf{L}} \otimes-}{\longrightarrow} \mathbf{D}(\mathrm{Q} \operatorname{coh} / X) \stackrel{\mathbf{R} f_{*}}{\longrightarrow} \mathbf{D}(\mathrm{Q} \operatorname{coh} / Y)
$$

takes $\varphi$ to an isomorphism.

To do this, observe the isomorphisms

$$
\begin{aligned}
\mathbf{R} f_{*}\left[\mathcal{E}^{\mathbf{L}} \otimes \mathbf{L} f^{*}\left(\prod_{\lambda \in \Lambda} y_{\lambda}\right)\right] \cong & \\
\cong \mathbf{R} f_{*} \mathcal{E}^{\mathbf{L}} \otimes\left(\prod_{\lambda \in \Lambda} y_{\lambda}\right) & \text { projection formula } \\
\cong \prod_{\lambda \in \Lambda}\left(\mathbf{R} f_{*} \mathcal{E}^{\mathbf{L}} \otimes y_{\lambda}\right) & \mathbf{R} f_{*} \mathcal{E} \text { is compact } \\
\cong \prod_{\lambda \in \Lambda} \mathbf{R} f_{*}\left(\mathcal{E}^{\mathbf{L}} \otimes \mathbf{L} f^{*} y_{\lambda}\right) & \text { projection formula } \\
\cong \mathbf{R} f_{*} \prod_{\lambda \in \Lambda}\left(\mathcal{E}^{\mathbf{L}} \otimes \mathbf{L} f^{*} y_{\lambda}\right) & \mathbf{R} f_{*} \text { has left adjoint } \\
& \cong \mathbf{R} f_{*}\left[\mathcal{E}^{\mathbf{L} \otimes} \prod_{\lambda \in \Lambda} \mathbf{L} f^{*} y_{\lambda}\right] \quad \mathcal{E} \text { is compact . }
\end{aligned}
$$

Note that in this string of isomorphisms we twice appealed to Reminder 2.7(iii); tensor product with a compact object commutes with products. We used it for the compact object $\varepsilon \in \mathbf{D}(\mathrm{Q} \operatorname{coh} / X)$ and for $\mathbf{R} f_{*} \mathcal{E} \in \mathbf{D}(\mathrm{Q} \operatorname{coh} / Y)$.

Remark 4.3. The fact that $\mathbf{L} f^{*}$ respects products means that it must have a left adjoint. Van den Bergh suggested a formula for this adjoint; it should be given by the functor $\mathbf{R} f_{*}\left[f^{!} \mathcal{O}_{Y} \mathbf{L}_{\otimes}-\right]$. Lipman and Van den 
Bergh independently found proofs that the formula works, at least for large classes of $f \mathrm{~s}$.

Lemma 4.4. Assume $f: X \longrightarrow Y$ is a morphism of quasicompact, separated schemes, and suppose further that $\mathbf{R} f_{*}$ takes compact objects in $\mathbf{D}(\mathrm{Qcoh} / X)$ to compact objects in $\mathbf{D}(\mathrm{Qcoh} / Y)$. Suppose $\left\{X_{\lambda}, \lambda \in \Lambda\right\}$ is a set of objects in $\mathbf{D}(\mathrm{Qcoh} / X)$, with each $X_{\lambda}$ isomorphic to a coproduct of suspensions of $\mathcal{O}_{X}$. Then the natural map

$$
f^{!} \mathcal{O}_{Y} \mathbf{L}_{\otimes}\left(\prod_{\lambda \in \Lambda} x_{\lambda}\right) \longrightarrow \prod_{\lambda \in \Lambda}\left(f^{!} \mathcal{O}_{Y} \mathbf{L}_{\otimes} x_{\lambda}\right)
$$

is an isomorphism.

Proof. We know that $\mathbf{L} f^{*} \Sigma^{n} \mathcal{O}_{Y} \cong \Sigma^{n} \mathcal{O}_{X}$; hence any suspension of $\mathcal{O}_{X}$ can be expressed as $\mathbf{L} f^{*}$ of some object of $\mathbf{D}(\mathrm{Qcoh} / Y)$, and so can any coproduct of suspensions. For each of our objects $X_{\lambda} \in \mathbf{D}(\mathrm{Qcoh} / X)$ we may choose an object $y_{\lambda} \in \mathbf{D}(Q \operatorname{coh} / Y)$ and an isomorphism $x_{\lambda} \cong \mathbf{L} f^{*} y_{\lambda}$. Now [51, Theorem 5.4] gives us a natural isomorphism, for every $y \in \mathbf{D}(\mathrm{Qcoh} / Y)$,

$$
f^{!} y \cong f^{!} \mathcal{O}_{Y} \mathbf{L}_{\otimes} \mathbf{L} f^{*} y,
$$

and we therefore have isomorphisms

$$
\begin{array}{rlrl}
f^{!} \mathcal{O}_{Y} \mathbf{L}_{\otimes}\left(\prod_{\lambda \in \Lambda} x_{\lambda}\right) & \cong f^{!} \mathcal{O}_{Y} \mathbf{L}_{\otimes}\left(\prod_{\lambda \in \Lambda} \mathbf{L} f^{*} y_{\lambda}\right) & \\
& \cong f^{!} \mathcal{O}_{Y} \mathbf{L} \mathbf{L} f^{*}\left(\prod_{\lambda \in \Lambda} y_{\lambda}\right) & \text { Lemma } 4.2 \\
& \cong f^{!}\left(\prod_{\lambda \in \Lambda} y_{\lambda}\right) & \quad[51, \text { Theorem 5.4] } \\
& \cong \prod_{\lambda \in \Lambda} f^{!} y_{\lambda} \\
& \cong \prod_{\lambda \in \Lambda}\left(f^{!} \mathcal{O}_{Y} \mathbf{L}_{\otimes} \mathbf{L} f^{*} y_{\lambda}\right) & \\
& \cong \prod_{\lambda \in \Lambda}\left(f^{!} \mathcal{O}_{Y} \mathbf{L}_{\otimes} x_{\lambda}\right),
\end{array}
$$

completing the proof. 
Remark 4.5. We have shown that $f^{!} \mathcal{O}_{Y}$ is an object of $\mathbf{D}(\mathrm{Qcoh} / X)$ so that the functor $f^{!} \mathcal{O}_{Y} \mathbf{L}_{\otimes}$ - commutes with some products; it commutes with products of the $X_{\lambda}$ s of Lemma 4.4. It is natural to ask whether it commutes with all products. Reminder 2.7(iii) tells us that this is equivalent to asking whether $f^{!} \mathcal{O}_{Y}$ is compact.

The general answer is No: we remind the reader how construct a counterexample. Consider simple case where $Y=\operatorname{Spec}(k)$, where $k$ is a field, and $f: X \longrightarrow Y$ is a finite morphism. For our choice of $Y$ the sheaf $\mathcal{O}_{Y}$ is a dualizing complex on $Y$ and Lemma 3.18, coupled with Theorem 3.14, tell us that $f^{!} O_{Y}$ is a dualizing complex on $X$. Our hypothesis is that $X$ is finite over $Y=\operatorname{Spec}(k)$, in which case we know that the (essentially unique) dualizing complex has finite injective dimension. If it is also a compact object then the complex $\mathbf{R} \mathcal{H} \operatorname{Com}\left(f^{!} \mathcal{O}_{Y}, f^{!} \mathcal{O}_{Y}\right)$ is quasi-isomorphic to the complex of homomorphisms from a bounded complex of finitely generated projectives to a bounded complex of injectives; but Proposition 3.6(ii) gives a quasiisomorphism $\mathcal{O}_{X} \longrightarrow \mathbf{R} \mathcal{H} \operatorname{Com}\left(f^{!} \mathcal{O}_{Y}, f^{!} \mathcal{O}_{Y}\right)$. This can only happen if $\mathcal{O}_{X}$ has a bounded injective resolution, that is if $X$ is a Gorenstein scheme.

Remark 4.6. While $f^{!} \bigcirc_{Y}$ need not in general be compact, there are interesting things one can say about it, facts which I do not fully understand. To illustrate one of the strange features, a phenomenon which seems mysterious to me, consider the following. By [51, Theorem 5.4] there is an isomorphism $f^{!}(-) \cong f^{!} \mathcal{O}_{Y} \mathbf{L} \otimes \mathbf{L} f^{*}(-)$. Applying $\mathbf{R} f_{*}$ to this isomorphism, and using the projection formula, we deduce an isomorphism

$$
\mathbf{R} f_{*} f^{!}(-) \cong\left[\mathbf{R} f_{*} f^{!} \mathcal{O}_{Y}\right]^{\mathbf{L}_{\otimes}-} .
$$

The functor on the left is the composite of two right adjoints, hence commutes with products. It follows that so does the functor on the right; Reminder 2.7(iii) now informs us that $\mathbf{R} f_{*} f^{!} \mathcal{O}_{Y}$ has to be compact. Even though $f^{!} \mathcal{O}_{Y}$ need not be compact, its pushforward $\mathbf{R} f_{*} f^{!} \mathcal{O}_{Y}$ must be.

In the light of Lemma 4.4 and Remark 4.5 it makes sense to study the class of objects $\mathcal{L} \in \mathbf{D}(\mathrm{Qcoh} / X)$ for which the functor $\mathcal{L}^{\mathbf{L}} \otimes$ - commutes with the products of Lemma 4.4. Let us make this a definition.

Definition 4.7. Let $X$ be a quasicompact, separated scheme. We define a class of objects $\mathcal{S}(X) \subset \mathbf{D}(\mathrm{Qcoh} / X)$ by the following property. An object $\mathcal{L} \in \mathbf{D}(\mathrm{Qcoh} / X)$ belongs to $\mathcal{S}(X)$ if and only if the natural map

$$
\mathcal{L}^{\mathbf{L}} \otimes\left(\prod_{\lambda \in \Lambda} x_{\lambda}\right) \longrightarrow \prod_{\lambda \in \Lambda}\left(\mathcal{L}^{\mathbf{L}} \otimes X_{\lambda}\right)
$$


is an isomorphism whenever $\left\{X_{\lambda}, \lambda \in \Lambda\right\}$ is a set of objects in $\mathbf{D}(\mathrm{Qcoh} / X)$ as in Lemma 4.4. We remind the reader: this means that each $X_{\lambda}$ isomorphic to a coproduct of suspensions of $\mathcal{O}_{X}$.

Remark 4.8. When $X$ can be understood from context we will omit it from the notation; that is we will write $\mathcal{S}$ for $\mathcal{S}(X)$. Reminder 2.7(iii) tells us that all compact objects belong to $\mathcal{S}=\mathcal{S}(X)$. Lemma 4.4 says that, if $f: X \longrightarrow Y$ is a morphism where $\mathbf{R} f_{*}$ takes compacts to compacts, then $f^{!} \mathcal{O}_{Y}$ belongs to $\mathcal{S}(X)$. Clearly, if $\mathcal{E} \longrightarrow \mathcal{F} \longrightarrow \mathcal{G} \longrightarrow \Sigma \mathcal{E}$ is a distinguished triangle, and if two of the objects belong to $\mathcal{S}$, then so does the third. Not so trivial is the lemma

Lemma 4.9. Suppose $\mathcal{E}$ is a compact object in $\mathbf{D}(\mathrm{Qcoh} / X)$, and let $\mathcal{L}$ be an object in the class $\mathcal{S}$ of Definition 4.7. Then there exists a compact object $\mathcal{F} \in \mathbf{D}(\mathrm{Qcoh} / X)$, and a morphism $f: \mathcal{F} \longrightarrow \mathcal{L}$, so that any morphism $\Sigma^{n} \mathcal{E} \longrightarrow \mathcal{L}$ factors as $\Sigma^{n} \mathcal{E} \longrightarrow \mathcal{F} \stackrel{f}{\longrightarrow} \mathcal{L}$.

Proof. Let $\Lambda$ be the set of all morphisms $\mathcal{E} \longrightarrow \Sigma^{n} \mathcal{L}$. If $\lambda \in \Lambda$ is a morphism $\mathcal{E} \longrightarrow \Sigma^{n} \mathcal{L}$, put $X_{\lambda}=\Sigma^{n} \mathcal{O}_{X}$. Because $\mathcal{L}$ belongs to $\mathcal{S}$ we know that the natural map

$$
\mathcal{L}^{\mathbf{L}} \otimes\left(\prod_{\lambda \in \Lambda} x_{\lambda}\right) \longrightarrow \prod_{\lambda \in \Lambda}\left(\mathcal{L}^{\mathbf{L}} \otimes x_{\lambda}\right)
$$

is an isomorphism. The collection $\Lambda$, of all maps $\mathcal{E} \longrightarrow \Sigma^{n} \mathcal{L}=\mathcal{L}^{\mathbf{L}} \otimes X_{\lambda}$, assembles to a single map

$$
\mathcal{E} \longrightarrow \prod_{\lambda \in \Lambda}\left(\mathcal{L}^{\mathbf{L}} \otimes X_{\lambda}\right)
$$

which must therefore factor as

$$
\mathcal{E} \longrightarrow \mathcal{L}^{\mathbf{L}} \otimes\left(\prod_{\lambda \in \Lambda} x_{\lambda}\right) \longrightarrow \prod_{\lambda \in \Lambda}\left(\mathcal{L}^{\mathbf{L}} \otimes X_{\lambda}\right) .
$$

But $\mathcal{E}$ is compact; any map $\mathcal{E} \longrightarrow \mathcal{G}^{\mathbf{L}} \otimes \mathcal{H}$ factors as $\mathcal{E} \longrightarrow \mathcal{F}^{\mathbf{L}} \otimes \mathcal{H} \stackrel{f^{\mathbf{L}} \otimes 1}{\longrightarrow}$ $\mathcal{G}^{\mathbf{L}} \otimes \mathcal{H}$, with $\mathcal{F} \in \mathbf{D}(\mathrm{Qcoh} / X)$ compact. Applying this to the above we deduce a factorization

$$
\mathcal{E} \longrightarrow \mathcal{F}^{\mathbf{L}} \otimes\left(\prod_{\lambda \in \Lambda} x_{\lambda}\right) \stackrel{f^{\mathbf{L}} \otimes 1}{\longrightarrow} \mathcal{L}^{\mathbf{L}} \otimes\left(\prod_{\lambda \in \Lambda} x_{\lambda}\right) \longrightarrow \prod_{\lambda \in \Lambda}\left(\mathcal{L}^{\mathbf{L}} \otimes X_{\lambda}\right) .
$$


Now consider the commutative diagram

$$
\begin{aligned}
& \mathcal{E} \longrightarrow \mathcal{F}^{\mathbf{L}} \otimes\left(\prod_{\lambda \in \Lambda} x_{\lambda}\right) \stackrel{f^{\mathbf{L}} \otimes 1}{\longrightarrow} \mathcal{L}^{\mathbf{L}} \otimes\left(\prod_{\lambda \in \Lambda} x_{\lambda}\right) \\
& \downarrow \\
& \prod_{\lambda \in \Lambda}\left(\mathcal{F}^{\mathbf{L}} \otimes X_{\lambda}\right) \stackrel{\downarrow}{\prod_{\lambda \in \Lambda} f^{\mathbf{L}} \otimes 1} \prod_{\lambda \in \Lambda}\left(\mathcal{L}^{\mathbf{L}} \otimes x_{\lambda}\right) .
\end{aligned}
$$

By construction, the composite from top left to bottom right amounts to assembling the collection of all maps $\mathcal{E} \longrightarrow \Sigma^{n} \mathcal{L}$ into a single morphism. The factorization

$$
\begin{gathered}
\mathcal{E} \longrightarrow \mathcal{F}^{\mathbf{L}} \otimes\left(\prod_{\lambda \in \Lambda} x_{\lambda}\right) \\
\downarrow \prod_{\lambda \in \Lambda}\left(\mathcal{F}^{\mathbf{L}} \otimes x_{\lambda}\right) \underset{\prod_{\lambda \in \Lambda} f^{\mathbf{L}} \otimes 1}{\longrightarrow} \prod_{\lambda \in \Lambda}\left(\mathcal{L}^{\mathbf{L}} \otimes x_{\lambda}\right)
\end{gathered}
$$

gives us a single map $f: \mathcal{F} \longrightarrow \mathcal{L}$, and informs us that each morphism $\mathcal{E} \longrightarrow \Sigma^{n} \mathcal{L}$ has a factorization $\mathcal{E} \longrightarrow \Sigma^{n} \mathcal{F} \stackrel{\Sigma^{n} f}{\longrightarrow} \Sigma^{n} \mathcal{L}$.

This already permits us to deduce the following two facts.

Lemma 4.10. Let $X$ be a quasicompact, separated scheme. Let $\mathcal{L}$ be an object belonging to $\mathcal{S}(X) \subset \mathbf{D}(\mathrm{Q} c o h / X)$. Then

(i) The object $\mathcal{L}$ is $\aleph_{1}$-compact, in the sense of [52, Definition 4.2.7].

(ii) If $\mathbf{D}(\mathrm{Qcoh} / X)$ has a strong compact generator then $\mathcal{L}$ is compact.

Proof. By [5, Theorem 3.1.1] there is a compact generator in $\mathbf{D}(\mathrm{Qcoh} / X)$; let $\mathcal{E}$ be such a compact generator. If we are proving (ii), assume further that $\mathcal{E}$ is a strong generator; this means that there exists an integer $n$ so that any compact can be obtained from $\mathcal{E}$ in $n$ steps; see [5, Definition 2.2.3].

Lemma 4.4 tells us that we may choose a compact object $\mathcal{F}=\mathcal{F}_{0}$, and a morphism $f_{0}: \mathcal{F}_{0} \longrightarrow \mathcal{L}$, so that any morphism $\Sigma^{n} \mathcal{E} \longrightarrow \mathcal{L}$ factors through $f_{0}$. That is, for every integer $n$ the functor $\operatorname{Hom}\left(\Sigma^{n} \mathcal{E},-\right)$ takes $f_{0}: \mathcal{F}_{0} \longrightarrow$ $\mathcal{L}$ to an epimorphism. Now assume we have a compact object $\mathcal{F}_{i}$ and a morphism $f_{i}: \mathcal{F}_{i} \longrightarrow \mathcal{L}$, and we will show how to produce a commutative triangle

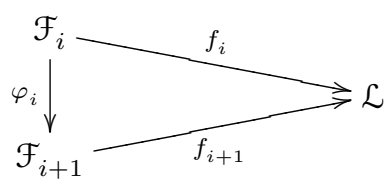


with the properties that

(iii) The object $\mathcal{F}_{i+1}$ is compact.

(iv) The group homomorphism $\operatorname{Hom}\left(\Sigma^{n} \mathcal{E}, \varphi_{i}\right)$ annihilates the kernel of $\operatorname{Hom}\left(\Sigma^{n} \mathcal{E}, f_{i}\right)$

Complete $f_{i}$ to a distinguished triangle

$$
\mathcal{L}^{\prime} \stackrel{g}{\longrightarrow} \mathcal{F}_{i} \stackrel{f_{i}}{\longrightarrow} \mathcal{L} \longrightarrow \Sigma \mathcal{L}^{\prime} .
$$

By assumption $\mathcal{L}$ belongs to $\mathcal{S}(X)$, as does the compact object $\mathcal{F}_{i}$. Therefore $\mathcal{L}^{\prime}$ also belongs to $\mathcal{S}(X)$. Applying Lemma 4.4 to $\mathcal{L}^{\prime}$ we discover a compact object $\mathcal{G}$ and a morphism $h: \mathcal{G} \longrightarrow \mathcal{L}^{\prime}$, so that every map $\Sigma^{n} \mathcal{E} \longrightarrow \mathcal{L}^{\prime}$ factors through $h$. That is, each of the functors $\operatorname{Hom}\left(\Sigma^{n} \mathcal{E},-\right)$ takes $h$ to a surjection. Since the functors $\operatorname{Hom}\left(\Sigma^{n} \mathcal{E},-\right)$ are homological they take any triangle to exact sequences; for every integer $n$ the functor $\operatorname{Hom}\left(\Sigma^{n} \mathcal{E},-\right)$ takes $\mathcal{L}^{\prime} \longrightarrow \mathcal{F}_{i} \longrightarrow \mathcal{L}$ to an exact sequence. Combining the surjections with the exact sequences, we deduce the exactness of

$\operatorname{Hom}\left(\Sigma^{n} \mathcal{E}, \mathcal{G}\right) \stackrel{\operatorname{Hom}\left(\Sigma^{n} \mathcal{E}, g h\right)}{\longrightarrow} \operatorname{Hom}\left(\Sigma^{n} \mathcal{E}, \mathcal{F}_{i}\right) \stackrel{\operatorname{Hom}\left(\Sigma^{n} \mathcal{E}, f_{i}\right)}{\longrightarrow} \operatorname{Hom}\left(\Sigma^{n} \mathcal{E}, \mathcal{L}\right)$. The kernel of $\operatorname{Hom}\left(\Sigma^{n} \mathcal{E}, f_{i}\right)$ is therefore the image of $\operatorname{Hom}\left(\Sigma^{n} \mathcal{E}, g h\right)$. Now complete $g h: \mathcal{G} \longrightarrow \mathcal{F}_{i}$ to a distinguished triangle

$$
\mathcal{G} \stackrel{g h}{\longrightarrow} \mathcal{F}_{i} \stackrel{\varphi_{i}}{\longrightarrow} \mathcal{F}_{i+1} \longrightarrow \Sigma \mathcal{G} \text {. }
$$

Clearly $\operatorname{Hom}\left(\Sigma^{n} \mathcal{E}, \varphi_{i}\right)$ annihilates the image of $\operatorname{Hom}\left(\Sigma^{n} \mathcal{E}, g h\right)$, which is the kernel of $\operatorname{Hom}\left(\Sigma^{n} \mathcal{E}, f_{i}\right)$; we have achieved (iv). Because $\mathcal{G}$ and $\mathcal{F}_{i}$ are both compact, so is $\mathcal{F}_{i+1}$; that is (iii) also holds. Because the composite $\mathcal{G} \stackrel{g h}{\longrightarrow} \mathcal{F}_{i} \stackrel{f_{i}}{\longrightarrow} \mathcal{L}$ vanishes, the map $f_{i}$ must factor as $\mathcal{F}_{i} \stackrel{\varphi_{i}}{\longrightarrow} \mathcal{F}_{i+1} \stackrel{f_{i+1}}{\longrightarrow} \mathcal{L}$. This yields our commutative triangle

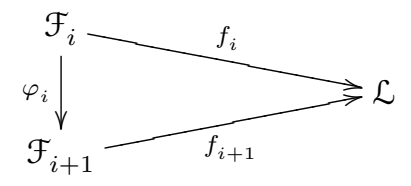

We have proved all our inductive claims.

We have a sequence of morphisms

$$
\mathcal{F}_{0} \stackrel{\varphi_{0}}{\longrightarrow} \mathcal{F}_{1} \stackrel{\varphi_{1}}{\longrightarrow} \mathcal{F}_{2} \stackrel{\varphi_{2}}{\longrightarrow} \cdots
$$

and compatible maps to $\mathcal{L}$; we can factor through a map

$$
\varphi: \underset{\text { Hocolim }}{\longrightarrow} \mathcal{F}_{i} \longrightarrow \mathcal{L} .
$$

The usual argument, as in Brown's original proof of the Brown representability theorem, tells us that each of the functors $\operatorname{Hom}\left(\Sigma^{n} \mathcal{E},-\right)$ takes the map $\varphi$ to an isomorphism; the reader can find this many places, for example in 
$[51, \S 3]$. Since $\mathcal{E}$ generates it follows that $\varphi$ is an isomorphism; $\mathcal{L}$ is isomorphic to Hocolim $f_{i}$, a countable homotopy colimit of compact objects. Hence $\mathcal{L}$ is $\aleph_{1}$-compact. This establishes (i).

It remains to prove (ii); from now we assume $\mathcal{E}$ is a strong generator, and our notation will be as in $[5, \S 2.2]$. Let $n$ be an integer so that $\langle\mathcal{E}\rangle_{n}=$ $\{\mathbf{D}(\mathrm{Qcoh} / X)\}^{c}$. Recall our sequence above, of morphisms $f_{i}: \mathcal{F}_{i} \longrightarrow \mathcal{L}$. One can show, by an induction on $i$ which we leave to the reader,

(v) Any morphism $\mathcal{K} \longrightarrow \mathcal{L}$, with $\mathcal{K} \in\langle\mathcal{E}\rangle_{i}$, can be factored through $f_{i}: \mathcal{F}_{i} \longrightarrow \mathcal{L}$.

(vi) Given a vanishing composite $\mathcal{K} \longrightarrow \mathcal{F}_{j} \stackrel{f_{j}}{\longrightarrow} \mathcal{L}$, with $\mathcal{K} \in\langle\mathcal{E}\rangle_{i}$, then the composite

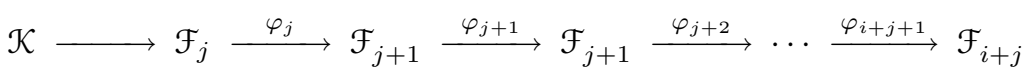
already vanishes.

In our sequence we constructed a morphism $f_{2 n}: \mathcal{F}_{2 n} \longrightarrow \mathcal{L}$, with $\mathcal{F}_{2 n}$ compact, that is with $\mathcal{F}_{2 n} \in\langle\mathcal{E}\rangle_{n}$. By (v) it must factor through $f_{n}: \mathcal{F}_{n} \longrightarrow$ $\mathcal{L}$. Choose a factorization $\mathcal{F}_{2 n} \stackrel{\alpha}{\longrightarrow} \mathcal{F}_{n} \stackrel{f_{n}}{\longrightarrow} \mathcal{L}$. The longer composite $\mathcal{F}_{n} \stackrel{\varphi}{\longrightarrow}$ $\mathcal{F}_{2 n} \stackrel{\alpha}{\longrightarrow} \mathcal{F}_{n} \stackrel{f_{n}}{\longrightarrow} \mathcal{L}$ is clearly equal to $f_{n}: \mathcal{F}_{n} \longrightarrow \mathcal{L}$. Put $e=1-\alpha \varphi$. Then $e: \mathcal{F}_{n} \longrightarrow \mathcal{F}_{n}$ is a morphism so that the composite $\mathcal{F}_{n} \stackrel{e}{\longrightarrow} \mathcal{F}_{n} \stackrel{f_{n}}{\longrightarrow} \mathcal{L}$ vanishes. By (vi) we deduce the vanishing of the composite $\mathcal{F}_{n} \stackrel{e}{\longrightarrow} \mathcal{F}_{n} \stackrel{\varphi}{\longrightarrow}$ $\mathcal{F}_{2 n}$. This means that the longer composite $\mathcal{F}_{n} \stackrel{e}{\longrightarrow} \mathcal{F}_{n} \stackrel{\varphi}{\longrightarrow} \mathcal{F}_{2 n} \stackrel{\alpha}{\longrightarrow} \mathcal{F}_{n}$ also vanishes; that is $e(1-e)=0$. We have shown that $e$ is an idempotent.

Therefore $\mathcal{F}_{n}$ splits as $\mathcal{F}_{n} \cong \mathcal{F} \oplus \mathcal{F}^{\prime}$, where $\alpha \varphi=1$ on $\mathcal{F}$ and 0 on $\mathcal{F}^{\prime}$. The reader can now check that each of the functors $\operatorname{Hom}\left(\Sigma^{n} \mathcal{E},-\right)$ takes the map $\mathcal{F} \longrightarrow \mathcal{L}$ to an isomorphism, and we conclude that $\mathcal{L} \cong \mathcal{F}$ is compact.

Remark 4.11. From Lemma 4.10(i) we learn that $\mathcal{S}(X) \subset\{\mathbf{D}(\mathrm{Q} \operatorname{coh} / X)\}^{\aleph_{1}}$, where $\{\mathbf{D}(\mathrm{Qcoh} / X)\}^{\aleph_{1}}$ is the full subcategory of $\aleph_{1}$-compact objects in $\mathbf{D}(\mathrm{Qcoh} / X)$. By $[52,8.4 .2 .2]$ we know that the category $\{\mathbf{D}(\mathrm{Q} c o h / X)\}^{\aleph_{1}}$ is essentially small. We deduce that, up to isomorphisms, there is only a set of objects in $\mathcal{S}(X)$.

Lemma 4.12. Let $X=\operatorname{Spec}(R)$ be an affine scheme. If $\mathcal{L}$ belongs to $\mathcal{S}(X)$ then $\mathcal{L}$ must be isomorphic in $\mathbf{D}(\mathrm{Qcoh} / X) \cong \mathbf{D}(R-\mathrm{Mod})$ to a bounded above complex of finitely generated projectives, and only finitely many of the cohomology groups of $\mathcal{L}$ can be non-zero.

Proof. We apply Lemma 4.9 with $\mathcal{E}=R$. We discover that there must exist a single compact object $\mathcal{F}$, that is a bounded complex of finitely generated, projective $R$-modules, as well single map $f: \mathcal{F} \longrightarrow \mathcal{L}$, so that any morphism $\Sigma^{n} R \longrightarrow \mathcal{L}$ factors through $f$. The morphisms $\Sigma^{n} R \longrightarrow \mathcal{L}$ are in bijection 
with elements of $H^{-n}(\mathcal{L})$ and we learn that, for every $i$, the map $H^{i}(\mathcal{F}) \longrightarrow$ $H^{i}(\mathcal{L})$ must be surjective. But $\mathcal{F}$ is bounded, hence $H^{i}(\mathcal{L})$ vanishes for all but finitely many $i$. It remains to show that $\mathcal{L}$ is isomorphic in $\mathbf{D}(R-\operatorname{Mod})$ to a bounded above chain complex of finitely generated projectives.

Suppose that $i$ is the integer at which the cohomology of $\mathcal{L}$ stops; that is $H^{i}(\mathcal{L}) \neq 0$, but $H^{j}(\mathcal{L})=0$ for all $j>i$. It suffices to produce a finitely generated, projective module $P^{i}$ and an epimorphism $P^{i} \longrightarrow H^{i}(\mathcal{L})$. Let us first establish that this really suffices. Suppose such a morphism exists; it must lift to a map in the derived category $\Sigma^{-i} P^{i} \longrightarrow \mathcal{L}=\mathcal{L}_{0}$. The third edge of the triangle $\Sigma^{-i} P^{i} \longrightarrow \mathcal{L} \longrightarrow \mathcal{L}_{1} \longrightarrow \Sigma^{-i+1} P^{i}$ is an object $\mathcal{L}_{1} \in \mathcal{S}(X)$ whose cohomology stops at $(i-1)$. We can now apply induction to obtain a sequence of finitely generated projectives and morphisms $\Sigma^{-k} P^{k} \longrightarrow \mathcal{L}_{k}$, where the cohomology of $\mathcal{L}_{k}$ stops at $i-k$, and these finitely generated projectives assemble to a chain complex

$$
\cdots \longrightarrow P^{i-2} \longrightarrow P^{i-1} \longrightarrow P^{i} \longrightarrow 0
$$

quasi-isomorphic to $\mathcal{L}$.

It therefore remains to prove the existence of the surjection $P^{i} \longrightarrow$ $H^{i}(\mathcal{L})$. Let $\mathcal{P}$ be a $K$-projective resolution for $\mathcal{L}$; we may choose $\mathcal{P}$ to be a bounded above complex of projective modules, which stops at $i$. That is $\mathcal{P}$ is a chain complex

$$
\cdots \longrightarrow \mathcal{P}^{i-2} \longrightarrow \mathcal{P}^{i-1} \longrightarrow \mathcal{P}^{i} \longrightarrow 0
$$

and $H^{i}(\mathcal{P}) \cong H^{i}(\mathcal{L}) \neq 0$. The morphism $f: \mathcal{F} \longrightarrow \mathcal{P}$ is a map in the derived category between bounded above chain complexes of projectives, and hence may be realized by a chain map. That is, we have a chain map
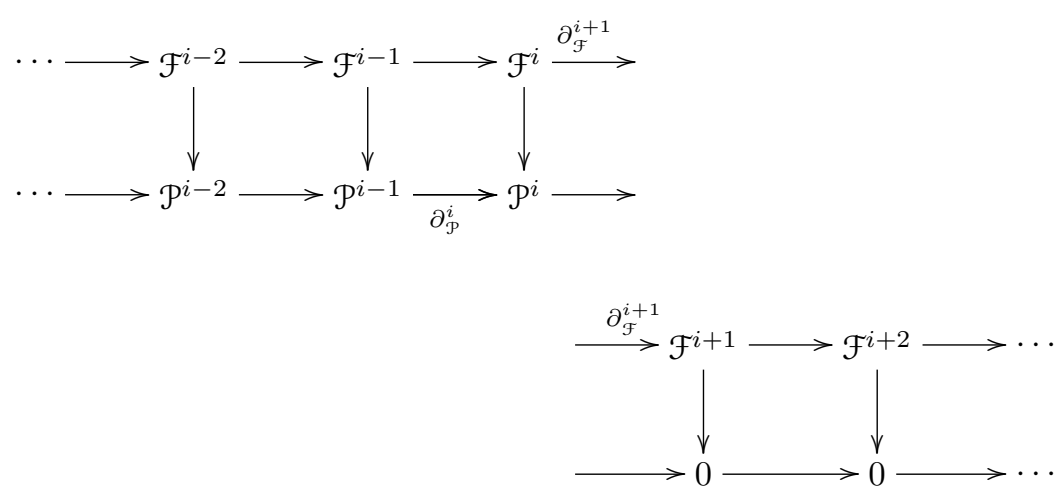

From the first paragraph of the proof we know that the map $H^{i}(\mathcal{F}) \longrightarrow$ $H^{i}(\mathcal{P})$ is surjective; that is the kernel of $\partial_{\mathcal{F}}^{i+1}$ surjects onto the cokernel of 
$\partial_{\mathcal{P}}^{i}$. But then $\mathcal{F}^{i}$ certainly must also surject to the cokernel; we have found our surjection $\mathcal{F}^{i} \longrightarrow H^{i}(\mathcal{P})$, with $\mathcal{F}_{i}$ finitely generated and projective.

This ends what I can currently prove in glorious generality. Let me next formulate a conjecture.

Conjecture 4.13. Let $X$ be any quasicompact, separated scheme, and let $\mathcal{L}$ be an object in $\mathcal{S}(X)$. Then $\mathcal{L}$ is pseudocoherent, and has only finitely many non-vanishing cohomology groups.

Reminder 4.14. We remind the reader: a complex $\mathcal{L}$ is pseudocoherent provided the restriction of $\mathcal{L}$, to any open affine subset $U \subset X$, is isomorphic in $\mathbf{D}(\mathrm{Q} c o h / U) \cong \mathbf{D}(R-\mathrm{Mod})$ to a bounded above complex of finitely generated projective $R$-modules.

Remark 4.15. In view of Lemma 4.12, Conjecture 4.13 amounts to the statement that if $\mathcal{L} \in \mathcal{S}(X)$ and $j: U \longrightarrow X$ is an open immersion of an affine open subset, then $j^{*}(\mathcal{L})$ is in $\mathcal{S}(U)$.

Let us formulate an even stronger conjecture:

Conjecture 4.16. Let $X$ be a quasicompact, separated scheme, and let $U \subset X$ be a quasicompact open subset. Let $j: U \longrightarrow X$ be the inclusion. Let the notation be as in [5, Definition 2.2.3]. There exists a compact object $\mathcal{E} \in \mathbf{D}(\mathrm{Qcoh} / X)$, and an integer $n \geq 1$, so that $\mathbf{R} j_{*} \mathcal{O}_{U} \in \overline{\langle\mathcal{L}\rangle_{n}}$.

Reminder 4.17. Perhaps we should remind the reader of the notation in [5, Definition 2.2.3]. Let $\mathcal{E}$ be an object in some triangulated category $\mathcal{T}$. The full subcategory $\overline{\langle\varepsilon\rangle_{1}}$ is defined to be the one containing all direct summands of all coproducts of arbitrary suspensions of $\mathcal{E}$. The category $\overline{\langle\mathcal{E}\rangle_{n}}$ is defined inductively; an object lies in $\overline{\langle\mathcal{E}\rangle}_{n+1}$ if it is a direct summand of an object $y$, and $y$ fits in a triangle

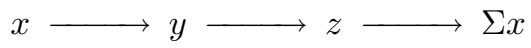

with $x \in \overline{\langle\mathcal{E}\rangle}_{1}$ and $z \in \overline{\langle\mathcal{L}\rangle}_{n}$.

Remark 4.18. Now that we have reminded the reader of the notation in Conjecture 4.16, we should also explain its relevance. In the remainder of this section we will show that Conjecture 4.16 implies Conjecture 4.13. In $\S 5$ we will study the many cases in which we know Conjecture 4.16 to be true. And then, in $\S 6$, we will return to the relation with Grothendieck duality. 
Definition 4.19. Let $X$ be a quasicompact, separated scheme. A class of objects $T \subset \mathbf{D}(\mathrm{Qcoh} / X)$ will be called adapted if the natural map

$$
\mathcal{L}^{\mathbf{L}} \otimes\left(\prod_{\lambda \in \Lambda} t_{\lambda}\right) \longrightarrow \prod_{\lambda \in \Lambda}\left(\mathcal{L}^{\mathbf{L}} \otimes t_{\lambda}\right)
$$

is an isomorphism, whenever the following conditions both hold:

(i) $\Lambda$ is a set of objects $\left\{t_{\lambda}, \lambda \in \Lambda\right\}$, where each $t_{\lambda}$ belongs to $T$.

(ii) The object $\mathcal{L}$ belongs to $\mathcal{S}(X)$.

Remark 4.20. By definition of $\mathcal{S}(X)$ the class $T$, containing all coproducts of arbitrary suspensions of $\mathcal{O}_{X}$, is an adapted class. If $T$ is an adapted class then so is

(i) The class of all mapping cones on morphisms $t \longrightarrow t^{\prime}$, with $t, t^{\prime} \in T$.

(ii) The class of all direct summands of objects in $T$.

(iii) The class of all objects $\mathcal{E}^{\mathbf{L}} \otimes t$, where $\mathcal{E}$ is a fixed compact object and $t \in T$.

All three facts are easy; we leave (i) and (ii) to the reader and indicate the proof of (iii). Let $\mathcal{E}$ be a compact object, $\mathcal{L}$ an object in $\mathcal{S}$, and assume all the $t_{\lambda}$ lie in $T$. We have isomorphisms

$$
\begin{aligned}
& 3 \mathcal{L}^{\mathbf{L}} \otimes\left[\prod_{\lambda \in \Lambda}\left(\mathcal{E}^{\mathbf{L}} \otimes t_{\lambda}\right)\right] \cong \mathcal{L}^{\mathbf{L}} \otimes \mathcal{E}^{\mathbf{L}} \otimes\left(\prod_{\lambda \in \Lambda} t_{\lambda}\right) \quad \text { Reminder 2.7(iii) } \\
& \cong \mathcal{E}^{\mathbf{L}} \otimes\left[\prod_{\lambda \in \Lambda}\left(\mathcal{L}^{\mathbf{L}} \otimes t_{\lambda}\right)\right] \quad \text { Because } \mathcal{L} \in \mathcal{S} \text {, all } t_{\lambda} \in T \\
& \cong \prod_{\lambda \in \Lambda}\left(\mathcal{L}^{\mathbf{L}} \otimes \mathcal{E}^{\mathbf{L}} \otimes t_{\lambda}\right) \quad \text { Reminder 2.7(iii) . }
\end{aligned}
$$

Lemma 4.21. Conjecture 4.16 implies Conjecture 4.13. More precisely for each X, Conjecture 4.16 for X implies Conjecture 4.13 for $X$. We spell this out: if $X$ is a quasicompact, separated scheme, and if Conjecture 4.16 holds for $X$, then every object $\mathcal{L} \in \mathcal{S}(X)$ is pseudocoherent, and the cohomology sheaves $\mathbb{H}^{i}(\mathcal{L})$ vanish for all but finitely many $i$.

Proof. Assume $X$ is a quasicompact, separated scheme, let $U \subset X$ be an affine open set, and let $j: U \longrightarrow X$ be the inclusion. We are assuming Conjecture 4.16 is true; we may choose a compact object $\mathcal{E} \in \mathbf{D}(\mathrm{Qcoh} / X)$, and an integer $n \geq 1$, so that $\mathbf{R} j_{*} \mathcal{O}_{U} \in \overline{\langle\mathcal{E}\rangle_{n}}$. Choose and fix $\mathcal{E}$ and $n$. Note that $j: U \longrightarrow X$ is an affine morphism, hence $j_{*}$ is exact; there is no difference between $\mathbf{R} j_{*} \mathcal{O}_{U}$ and $j_{*} \mathcal{O}_{U}$. We will use the shorter $j_{*} \mathcal{O}_{U}$.

We observed, at the beginning of Remark 4.20, that the class $T$ of all coproducts of all suspensions of $\mathcal{O}_{X}$ is adapted. By Remark 4.20(iii) so is 
the class of all $\mathcal{E}^{\mathbf{L}} \otimes t$, with $\mathcal{E}$ as in the previous paragraph and $t \in T$. That is, the class of all coproducts of arbitrary suspensions of $\mathcal{E}$ is adapted. By Remark 4.20(ii) so is the class of all direct summands of the above; that is $\overline{\langle\mathcal{E}}_{1}$ is adapted. Now parts (i) and (ii) of Remark 4.20 tell us that if $\overline{\langle\mathcal{L}\rangle_{i}}$ is adapted then so is $\overline{\langle\mathcal{E}}_{2 i}$. We conclude that all the classes ${\overline{\langle\mathcal{E}\rangle_{i}}}_{i}$ are adapted.

Now the first paragraph informs us that $\overline{\langle\mathcal{E}\rangle_{n}}$ contains all coproducts of arbitrary suspensions of $j_{*} \mathcal{O}_{U}$. Now $j_{*}$ respects coproducts; a coproduct of suspensions of $j_{*} \mathcal{O}_{U}$ is $j_{*}$ of the coproduct of suspensions of $\mathcal{O}_{U}$. Let $T^{\prime} \subset \mathbf{D}(\mathrm{Q} c o h / U)$ be the class of all coproducts of arbitrary suspensions of $\mathcal{O}_{U}$; we have that $j_{*} T^{\prime}$ is an adapted class. This means that the natural map

$$
\mathcal{L}^{\mathbf{L}} \otimes\left(\prod_{\lambda \in \Lambda} j_{*} t_{\lambda}\right) \longrightarrow \prod_{\lambda \in \Lambda}\left(\mathcal{L}^{\mathbf{L}} \otimes j_{*} t_{\lambda}\right)
$$

is an isomorphism, whenever the following conditions both hold:

(i) $\Lambda$ is a set of objects $\left\{t_{\lambda}, \lambda \in \Lambda\right\}$, where each $t_{\lambda}$ belongs to $T^{\prime}$.

(ii) The object $\mathcal{L}$ belongs to $\mathcal{S}(X)$.

Now take an object $\mathcal{L} \in \mathcal{S}(X)$ and a set of objects $\left\{t_{\lambda}, \lambda \in \Lambda\right\}$, where each $t_{\lambda}$ belongs to $T^{\prime}$, and observe the string of isomorphisms

$$
\begin{aligned}
& 3 j_{*}\left[j^{*} \mathcal{L}^{\mathbf{L}} \otimes\left(\prod_{\lambda \in \Lambda} t_{\lambda}\right)\right] \cong \mathcal{L}^{\mathbf{L}} \otimes j_{*}\left(\prod_{\lambda \in \Lambda} t_{\lambda}\right) \quad \text { projection formula } \\
& \cong \mathcal{L}^{\mathbf{L}} \otimes\left(\prod_{\lambda \in \Lambda} j_{*} t_{\lambda}\right) \quad j_{*} \text { has left adjoint } \\
& \cong \prod_{\lambda \in \Lambda}\left(\mathcal{L}^{\mathbf{L}} \otimes j_{*} t_{\lambda}\right) \quad j_{*} T^{\prime} \text { is adapted } \\
& \cong \prod_{\lambda \in \Lambda} j_{*}\left(j^{*} \mathcal{L}^{\mathbf{L}} \otimes t_{\lambda}\right) \quad \text { projection formula }
\end{aligned}
$$

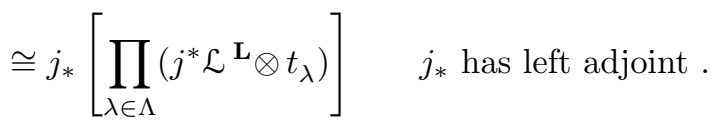


Applying $j^{*}$ to the isomorphism, and recalling that $j^{*} j_{*}$ is naturally isomorphic to the identity, this simplifies to saying that the natural map

$$
j^{*} \mathcal{L}^{\mathbf{L}_{\otimes}}\left(\prod_{\lambda \in \Lambda} t_{\lambda}\right) \longrightarrow \prod_{\lambda \in \Lambda}\left(j^{*} \mathcal{L} \mathbf{L}_{\otimes} t_{\lambda}\right)
$$

Is an isomorphism. That is $j^{*} \mathcal{L}$ belongs to $\mathcal{S}(U) \subset \mathbf{D}(\mathrm{Q} c o h / U)$. Since $U$ is affine, Lemma 4.12 tells us that $j^{*} \mathcal{L}$ is a bounded above complex of finitely generated projectives, with bounded cohomology.

\section{Where We CAN Prove Conjecture 4.16}

This section is about studying the cases where we know Conjecture 4.16 to be true. The conjecture is the assertion that sheaves of the form $\mathbf{R} j_{*} \mathcal{O}_{U}$ lie in $\overline{\langle\mathcal{E}\rangle_{n}}$, for suitable choices of a compact object $\mathcal{E}$ and an integer $n$. We observe

Remark 5.1. Let $X$ be a quasicompact, separated scheme. If there is a compact object $\mathcal{E} \in \mathbf{D}(\mathrm{Qcoh} / X)$ and an integer $n$ so that $\overline{\langle\mathcal{\langle}\rangle_{n}}=\mathbf{D}(\mathrm{Qcoh} / X)$, then Conjecture 4.16 most definitely holds for $X$.

And the next observation is that there are examples in the literature. Specifically, the reader is referred to the $\operatorname{proof}^{5}$ of $[5$, Theorem 3.1.4]. In there it is shown that, if $X$ is smooth over a field $k$, then $\overline{\langle\varepsilon\rangle_{n}}=\mathbf{D}(\mathrm{Qcoh} / X)$ for some $n$ and some compact $\mathcal{E}$. In Theorem 5.13 we will prove a very slight refinement of the result in [5]; there is no need to assume that the ground ring is a field, a finite dimensional, regular, noetherian ring is quite enough.

This means that we have cheap examples of schemes $X$ for which Conjecture 4.16 is true; but all of them are smooth and noetherian. To obtain singular, non-noetherian examples we will have to learn how to produce new examples out of old ones. This section is mostly about developing the machinery.

We begin with a little definition.

Definition 5.2. Let $X$ be a quasicompact, separated scheme. An open set $U \subset X$ will be called decent if

(i) $U$ is quasicompact.

(ii) Let $j: U \longrightarrow X$ be the inclusion. There exist a compact object $\mathcal{E} \in$ $\mathbf{D}(\mathrm{Qcoh} / X)$, and an integer $n \geq 1$, so that $\mathbf{R} j_{*} \mathcal{O}_{U} \in{\overline{\langle\mathcal{\langle}\rangle_{n}}}_{\text {. }}$.

\footnotetext{
${ }^{5}$ The reader should note that the statement of [5, Theorem 3.1.4] is slightly different; the result we want is merely a step in the proof. See the last paragraph of $[5, \S 3.4]$ for details.
} 
Remark 5.3. In the terminology introduced in Definition 5.2, Conjecture 4.16 asserts that every quasicompact open subset $U \subset X$ is decent. The idea of this section will be to find ways to produce decent open subsets.

Lemma 5.4. Let $X=\operatorname{Spec}(R)$ be an affine scheme, and let $X_{f}=\operatorname{Spec}(R[1 / f])$ be the basic open subset consisting of the prime ideals not containing $f \in R$. Then $X_{f} \subset X$ is decent.

Proof. Being affine, $X_{f}$ is clearly quasicompact. In the category

$$
\mathbf{D}(\mathrm{Q} \operatorname{coh} / X) \cong \mathbf{D}(R-\mathrm{Mod}),
$$

the object $\mathbf{R} j_{*} \mathcal{O}_{X_{f}}$ simplifies to $R[1 / f] \in \mathbf{D}(R-$ Mod $)$. We need find a compact object $\mathcal{E}$ and an integer $n$, and exhibit $R[1 / f]$ as an object in $\overline{\langle\mathcal{\langle}\rangle_{n}}$. Now note that $R[1 / f]$ is the colimit of the sequence

$$
R \stackrel{f}{\longrightarrow} R \stackrel{f}{\longrightarrow} R \stackrel{f}{\longrightarrow} R \stackrel{f}{\longrightarrow} R \stackrel{f}{\longrightarrow} \cdots
$$

It is therefore also the homotopy colimit. There is a triangle in $\mathbf{D}(R-$ Mod $)$

$$
\coprod_{n=0}^{\infty} R \longrightarrow \coprod_{n=0}^{\infty} R \longrightarrow R[1 / f] \longrightarrow \coprod_{n=0}^{\infty} \Sigma R \text {. }
$$

Put $\mathcal{E}=R$ and $n=2$; the triangle shows that $R[1 / f]$ belongs to ${\overline{\langle R\rangle_{2}}}_{2}$.

Lemma 5.5. Suppose $X$ is a quasicompact, separated scheme. Let $U$ and $V$ be open subsets, and assume that $U, V$ and $U \cap V$ are all decent. Then so is $U \cup V$.

Proof. The quasicompactness is clear; $U \cup V$ is the union of two quasicompact open sets $U$ and $V$. We have to worry about the sheaf $\mathbf{R} j_{*} \mathcal{O}_{U \cup V}$. Since there are several open subsets $W \subset X$ to consider, and several inclusions among them, we return to our notation of the proof of Lemma 2.4; given open subsets $W_{1} \subset W_{2}$ of $X$, we will denote the inclusion by $j_{W_{1}}^{W_{2}}: W_{1} \longrightarrow$ $W_{2}$.

By hypothesis we may choose an integer $n$, and compact objects $\mathcal{E}_{U}, \mathcal{E}_{V}$ and $\mathcal{E}_{U \cap V}$ in $\mathbf{D}(\mathrm{Q} \operatorname{coh} / X)$, so that

$$
\mathbf{R}\left\{j_{U}^{X}\right\}_{*} \mathcal{O}_{U} \in \overline{\left\langle\mathcal{E}_{U}\right\rangle_{n}}, \quad \mathbf{R}\left\{j_{V}^{X}\right\}_{*} \mathcal{O}_{V} \in \overline{\left\langle\mathcal{E}_{V}\right\rangle_{n}}, \quad \mathbf{R}\left\{j_{U \cap V}^{X}\right\}_{*} \mathcal{O}_{U \cap V} \in \overline{\left\langle\mathcal{E}_{U \cap V}\right\rangle_{n}} .
$$

Put $\mathcal{E}=\mathcal{E}_{U} \oplus \mathcal{E}_{V} \oplus \mathcal{E}_{U \cap V}$. Then $\mathcal{E}$ is compact, and the three complexes

$$
\mathbf{R}\left\{j_{U}^{X}\right\}_{*} \mathcal{O}_{U}, \quad \mathbf{R}\left\{j_{V}^{X}\right\}_{*} \mathcal{O}_{V}, \quad \mathbf{R}\left\{j_{U \cap V}^{X}\right\}_{*} \mathcal{O}_{U \cap V}
$$

all belong to $\overline{\langle\mathcal{E}\rangle}_{n}$. Now consider the distinguished triangle

$$
\begin{aligned}
\mathbf{R}\left\{j_{U \cup V}^{X}\right\}_{*} \mathcal{O}_{U \cup V} \longrightarrow \mathbf{R}\left\{j_{U}^{X}\right\}_{*} \mathcal{O}_{U} \oplus \mathbf{R}\left\{j_{V}^{X}\right\}_{*} \mathcal{O}_{V} \longrightarrow \\
\longrightarrow \\
\longrightarrow \mathbf{R}\left\{j_{U \cap V}^{X}\right\}_{*} \mathcal{O}_{U \cap V} \longrightarrow
\end{aligned}
$$


Two of the objects belong to $\overline{\langle\varepsilon\rangle}_{n}$ and hence the third, namely $\mathbf{R}\left\{j_{U \cup V}^{X}\right\}_{*} \mathcal{O}_{U \cup V}$, must belong to ${\overline{\langle\mathcal{E}\rangle_{2 n}}}_{2}$.

Lemma 5.6. Let $X=\operatorname{Spec}(R)$ be affine. Then any quasicompact open subset $U \subset X$ is decent. That is, Conjecture 4.16 holds for $X$.

Proof. The open sets $X_{f}, f \in R$ form a basis for the topology of $X=$ $\operatorname{Spec}(R)$; any open set $U$ is the union of the $X_{f}$ 's contained in it. If $U$ is quasicompact we may express it as a finite union of $X_{f}$ 's. We will prove, by induction on $n$,

(i) The union of $n$ basic open sets, that is open sets of the form $X_{f}$, is a decent open set.

Lemma 5.4 gives us the case $n=1$. Suppose therefore that we know (i) for $n$, that $U=\cup_{i=1}^{n} X_{f_{i}}$ is the union of $n$ open sets $X_{f_{i}}$, and that $V=X_{g}$ is another basic open set. We want to show that $U \cup V$ is decent, and Lemma 5.5 tells us that it suffices to show that $U, V$ and $U \cap V$ are. The case of $V=X_{g}$ comes from Lemma 5.4, while for $U$ and

$$
U \cap V=\left(\bigcup_{i=1}^{n} X_{f_{i}}\right) \cap X_{g}=\bigcup_{i=1}^{n}\left(X_{f_{i}} \cap X_{g}\right)=\bigcup_{i=1}^{n} X_{f_{i} g}
$$

we appeal to the fact that both can be covered by $n$ basic open sets.

Lemma 5.7. Decency is transitive. Precisely: suppose $X$ is a quasicompact, separated scheme, $U \subset X$ is a decent open set of $X$, and $V \subset U$ is a decent open subset of $U$. Then $V$ is a decent open subset of $X$.

Proof. The quasicompactness is clear. To keep the notation uncluttered let us write $j_{1}: V \longrightarrow U, j_{2}: U \longrightarrow X$ and $j: V \longrightarrow X$ for the three inclusions; we wish to prove that $\mathbf{R} j_{*} \mathcal{O}_{V}$ is contained in some $\overline{\langle\mathcal{K}\rangle_{\ell}}$.

Because $V$ is a decent open subset of $U$ we may produce a compact object $\mathcal{E} \in \mathbf{D}(\mathrm{Qcoh} / U)$, and an integer $m \geq 1$, so that $\mathbf{R}\left\{j_{1}\right\}_{*} \mathcal{O}_{V} \in \overline{\langle\mathcal{E}\rangle_{m}}$. The complex $\mathcal{E} \oplus \Sigma \mathcal{E}$ vanishes in the Grothendieck group $K_{0}(U)$, and Thomason's localization theorem $[62,5.2 .2(\mathrm{a})]$ applies. There exists a compact object $\mathcal{F} \in \mathbf{D}(\mathrm{Qcoh} / X)$ with $j_{2}^{*} \mathcal{F} \cong \mathcal{E} \oplus \Sigma \mathcal{E}$. Choose such an $\mathcal{F}$; we have that

$$
\mathbf{R}\left\{j_{1}\right\}_{*} \mathcal{O}_{V} \in{\overline{\left\langle j_{2}^{*} \mathcal{F}\right\rangle_{m}}} .
$$

We also know that $U$ is decent in $X$. We may therefore find a compact object $\mathcal{G} \in \mathbf{D}(\mathrm{Qcoh} / X)$, and an integer $n \geq 1$, so that $\mathbf{R}\left\{j_{2}\right\}_{*} \mathcal{O}_{U} \in \overline{\langle\mathcal{G}\rangle_{n}}$. Now we combine these. If we apply the functor $\mathbf{R}\left\{j_{2}\right\}_{*}$ to the inclusion $\left(^{*}\right)$ above, we conclude that the complex $\mathbf{R} j_{*} \mathcal{O}_{V}=\mathbf{R}\left\{j_{2}\right\}_{*} \mathbf{R}\left\{j_{1}\right\}_{*} \mathcal{O}_{V}$ is 
contained in

$$
\begin{array}{rlrl}
3 \mathbf{R}\left\{j_{2}\right\}_{*}{\overline{\left\langle j_{2}^{*} \mathcal{F}\right\rangle_{m}}}_{m} & \subset \overline{\left\langle\mathbf{R}\left\{j_{2}\right\}_{*} j_{2}^{*} \mathcal{F}\right\rangle_{m}} & & \mathbf{R}\left\{j_{2}\right\}_{*} \text { respects coproducts } \\
& =\overline{\left\langle\mathbf{R}\left\{j_{2}\right\}_{*}\left(j_{2}^{*} \mathcal{F} \mathbf{L} \otimes \mathcal{O}_{U}\right)\right\rangle_{m}} & & \text { obvious } \\
& =\overline{\left\langle\mathcal{F} \mathbf{L} \otimes \mathbf{R}\left\{j_{2}\right\}_{*} \mathcal{O}_{U}\right\rangle_{m}} & & \text { projection formula } \\
& \subset \overline{\langle\mathcal{F} \mathbf{L} \otimes \mathcal{G}\rangle_{m n}}, &
\end{array}
$$

where the last inclusion is because $\mathbf{R}\left\{j_{2}\right\}_{*} \mathcal{O}_{U}$ belongs to $\overline{\langle\mathcal{G}}_{n}$, hence $\mathcal{F}^{\mathbf{L}} \otimes \mathbf{R}\left\{j_{2}\right\}_{*} \mathcal{O}_{U}$ belongs to $\overline{\langle\mathcal{F} \mathbf{L} \otimes \mathcal{G}\rangle_{n}}$, and therefore

$$
\overline{\left\langle\mathcal{F} \mathbf{L} \otimes \mathbf{R}\left\{j_{2}\right\}_{*} \mathcal{O}_{U}\right\rangle_{m}} \subset \overline{\langle\mathcal{F} \mathbf{L} \otimes \mathcal{G}\rangle_{m n}} .
$$

Lemma 5.8. Let $X$ be a quasicompact, separated scheme, and let $\left\{U_{i}, 1 \leq\right.$ $i \leq n\}$ be a finite number of decent open affine subsets of $X$. Then the union $\cup_{i=1}^{n} U_{i}$ is decent.

Proof. We prove this by induction on $n$, the case $n=1$ being trivial. Suppose we know the assertion for $n$, and suppose we have $n+1$ decent open affines $\left\{U_{i}, 1 \leq i \leq n+1\right\}$. Let $U=\cup_{i=1}^{n} U_{i}$ and let $V=U_{n+1}$; by induction we know that $U$ and $V$ are decent, and we want to prove that so is $U \cup V$. By Lemma 5.5 it suffices to prove the decency of $U \cap V$. Now $X$ is assumed separated, hence the intersection of two quasicompact open subsets is quasicompact. Therefore $U \cap V \subset V$ is a quasicompact open subset of the affine scheme $V$, and Lemma 5.6 informs us that $U \cap V$ is decent in $V$. We are given that $V$ is decent in $X$ and the transitivity of decency, that is Lemma 5.7, permits us to conclude that $U \cap V$ is decent in $X$.

Proposition 5.9. Suppose $X$ is a quasicompact, separated scheme, and suppose that $X$ can be covered by decent open affines. Then Conjecture 4.16 holds for X; all quasicompact open sets are decent.

Proof. We are assuming that $X$ is quasicompact and has a cover by decent open affines. There must exist a finite subcover. We may cover $X$ by $n$ decent open affines, for some integer $n \geq 1$; the proof will be by induction on $n$. The case $n=1$ comes from Lemma 5.6. Suppose therefore that we know the assertion for schemes $X$ which admit covers by $n$ decent open affines, and let $X$ be a scheme admitting a cover by $n+1$ decent open affines. Then $X=U \cup V$, where $U$ has a cover by $n$ decent open affines and $V$ is a decent open affine. Lemma 5.8 informs us that $U \subset X$ is decent. Let $W \subset X$ be any quasicompact open subset; we want to prove that $W$ is decent. 
To do this observe that $W$ can be written as the union

$$
W=(W \cap U) \cup(W \cap V) .
$$

Now $X$ is assumed separated, and hence the intersection of any two quasicompact open sets is quasicompact; we deduce that $W \cap U, W \cap V$ and $W \cap U \cap V$ are all quasicompact. Induction tells us that

(i) $W \cap U$ is decent as an open subset of the scheme $U$.

(ii) $W \cap V$ and $W \cap U \cap V$ are both decent as open subsets of the scheme $V$.

Now the transitivity of decency, that is Lemma 5.7, guarantees that $W \cap U$, $W \cap V$ and $W \cap U \cap V$ are all decent as open subsets of $X$. Lemma 5.5 permits us to conclude that $W \subset X$ is decent.

Lemma 5.10. Let $f: X \longrightarrow Y$ be a morphism of quasicompact, separated schemes. If $V \subset Y$ is decent then so is $f^{-1} V \subset X$.

Proof. $Y$ is separated and the schemes $X$ and $V \subset Y$ are quasicompact, hence $f^{-1} V \subset X$ is quasicompact. The decency of $V \subset Y$ says further that we may choose a compact object $\mathcal{E} \in \mathbf{D}(\mathrm{Qcoh} / Y)$ and an integer $n \geq 1$ with $\mathbf{R}\left\{j_{V}^{Y}\right\}_{*} \mathcal{O}_{V} \in \overline{\langle\mathcal{E}\rangle_{n}}$. Therefore $\mathbf{L} f^{*} \mathcal{E}$ is a compact object in $\mathbf{D}(\mathrm{Qcoh} / X)$, and $\overline{\left\langle\mathbf{L} f^{*} \mathcal{E}\right\rangle_{n}}$ contains the object

$$
\mathbf{L} f^{*} \mathbf{R}\left\{j_{V}^{Y}\right\}_{*} \mathcal{O}_{V} \cong \mathbf{R}\left\{j_{f^{-1} V}^{X}\right\}_{*} \mathbf{L} f^{*} \mathcal{O}_{V} \cong \mathbf{R}\left\{j_{f^{-1} V}^{X}\right\}_{*} \mathcal{O}_{f^{-1} V} .
$$

Corollary 5.11. Let $f: X \longrightarrow Y$ be a morphism of quasicompact, separated schemes. Suppose $X$ can be covered by open affine subsets $f^{-1} V$, where $V \subset Y$ is open and quasicompact. If Conjecture 4.16 holds for $Y$ then it also holds for $X$.

Proof. Conjecture 4.16 holds for $Y$ and therefore any quasicompact $V \subset Y$ is decent. By Lemma 5.10 all the open sets $f^{-1} V \subset X$ are decent, and by hypothesis we may choose among them a collection of affines which cover $X$. Proposition 5.9 allows us to conclude that Conjecture 4.16 holds for $X$.

Remark 5.12. There are two useful situations where Corollary 5.11 applies. They are:

(i) Any open immersion of quasicompact, separated schemes.

(ii) Affine morphisms; for example closed immersions, or more generally finite maps.

So much for machinery of confirming the decency of open subsets. Now we come to the way of producing examples. 
Theorem 5.13. Let $R$ be a (noetherian) regular ring of finite global dimension, and assume $X$ is a noetherian, separated scheme, smooth and finite dimensional over $R$. There exists an integer $n \geq 1$, as well as a compact object $\mathcal{E} \in \mathbf{D}(\mathrm{Qcoh} / X)$, with $\overline{\langle\mathcal{E}}_{n}=\mathbf{D}(\mathrm{Q} \operatorname{coh} / X)$.

Proof. As we already mentioned, the proof is a miniscule modification of an argument that may be found in [5]. Consider the product $X \times_{R} X$. It is a finite dimensional, regular, noetherian scheme, and the sheaf $\mathcal{O}_{\Delta}$, that is the structure sheaf of the diagonal embedding $X \longrightarrow X \times_{R} X$, is a coherent sheaf on $X \times_{R} X$. Locally it has a finite resolution by finitely generated projectives; that is $\mathcal{O}_{\Delta}$ is a compact object in $\mathbf{D}\left(\mathrm{Q} \operatorname{coh} / X \times_{R} X\right)$.

Now choose a compact object $\mathcal{E}$ generating $\mathbf{D}(\mathrm{Q} \operatorname{coh} / X)$; such an object exists by [5, Theorem 3.1.1(2)]. From [5, Lemma 3.4.1] we learn that $\mathcal{F}=$ $\pi_{1}^{*} \mathcal{E} \mathbf{L} \otimes \pi_{2}^{*} \mathcal{E}$ is a compact generator of $\mathbf{D}\left(\mathrm{Q} \operatorname{coh} / X \times_{R} X\right)$. The compact object $\mathcal{O}_{\Delta}$ therefore lies in $\overline{\langle\mathcal{F}\rangle}_{n}$ for some integer $n \geq 1$. Also, because $R$ is a regular, finite dimensional ring, there is an integer $m$ with $\overline{\langle R\rangle}_{m}=$ $\mathbf{D}(R-$ Mod $)$. We assert that $\overline{\langle\mathcal{E}\rangle}_{m n}=\mathbf{D}(\mathrm{Q} \operatorname{coh} / X)$.

To prove this, choose any object $\mathcal{S} \in \mathbf{D}(\mathrm{Qcoh} / X)$. Then $\mathcal{S}$ can be expressed as

$$
\mathcal{S} \cong\left\{\pi_{1}\right\}_{*}\left[\mathcal{O}_{\Delta} \mathbf{L}_{\otimes}\left(\pi_{2}^{*} \mathcal{S}\right)\right] .
$$

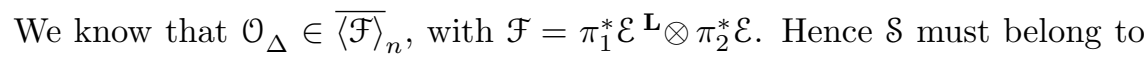
$\overline{\langle\mathcal{G}}_{n}$, where

$$
\mathcal{G} \cong\left\{\pi_{1}\right\}_{*}\left[\mathcal{F}^{\mathbf{L}} \otimes\left(\pi_{2}^{*} \mathcal{S}\right)\right] \cong\left\{\pi_{1}\right\}_{*}\left[\pi_{1}^{*} \mathcal{E}^{\mathbf{L}} \otimes \pi_{2}^{*} \mathcal{E}^{\mathbf{L}} \otimes\left(\pi_{2}^{*} \mathcal{S}\right)\right] .
$$

Manipulating a little more we have

$$
\mathcal{G} \cong\left\{\pi_{1}\right\}_{*}\left[\pi_{1}^{*} \mathcal{E}^{\mathbf{L}} \otimes \pi_{2}^{*}\left(\mathcal{E}^{\mathbf{L}} \otimes \mathcal{S}\right)\right] \cong \mathcal{E}^{\mathbf{L}} \otimes\left\{\pi_{1}\right\}_{*}\left[\pi_{2}^{*}\left(\mathcal{E}^{\mathbf{L}} \otimes \mathcal{S}\right)\right],
$$

and $\left\{\pi_{1}\right\}_{*}\left[\pi_{2}^{*}(\mathcal{E} \mathbf{L} \otimes \mathcal{S})\right] \cong \pi^{*} \pi_{*}(\mathcal{E} \mathbf{L} \otimes \mathcal{S})$ is $\pi^{*}$ of a complex $\pi_{*}\left(\mathcal{E}^{\mathbf{L}} \otimes \mathcal{S}\right)$ belonging to $\mathbf{D}(R$-Mod $)=\overline{\langle R\rangle}_{m}$. Thus $\pi^{*} \pi_{*}\left(\mathcal{E} \mathbf{L}_{\otimes} \mathcal{S}\right)$ must belong to $\overline{\left\langle\mathcal{O}_{X}\right\rangle}{ }_{m} \subset \mathbf{D}(\mathrm{Q} \operatorname{coh} / X)$, and

$$
\mathcal{G} \cong \mathcal{E}^{\mathbf{L}} \otimes\left[\pi^{*} \pi_{*}\left(\mathcal{E}^{\mathbf{L}} \otimes \mathcal{S}\right)\right]
$$

lies in $\overline{\langle\mathcal{E}\rangle}_{m}$. Hence $\mathcal{S} \in{\overline{\langle\mathcal{G}\rangle_{n}}}_{\overline{\langle\mathcal{E}\rangle}}$.

\section{Dualizing complexes and $f^{!}$}

Let us leave the world of gorgeous generality and return to dealing with more restricted schemes, at least some of which will be noetherian. Assume $f: X \longrightarrow Y$ is a morphism of quasicompact, separated schemes, suppose $Y$ is noetherian, and let $\mathcal{J}$ be a dualizing complex on $Y$. If $\mathcal{E}$ is any object 
in $\mathbf{D}(\mathrm{Q} \operatorname{coh} / X)$, and $\mathcal{F}$ belongs to $\mathbf{D}^{b}(\mathrm{Coh} / Y) \subset \mathbf{D}(\mathrm{Qcoh} / Y)$, then we compute

$$
\begin{aligned}
\operatorname{Hom}_{Y}\left(\mathbf{R} f_{*} \mathcal{E}, \mathcal{F}\right) & \cong \operatorname{Hom}_{Y}\left[\mathbf{R} f_{*} \mathcal{E}, \mathbf{R} \mathcal{H o m}_{Y}\left(\mathbf{R} \mathcal{H} \operatorname{Hom}_{Y}(\mathcal{F}, \mathcal{J}), \mathcal{J}\right)\right] \\
& \cong \operatorname{Hom}_{Y}\left[\mathbf{R} f_{*} \mathcal{E} \mathbf{L} \otimes \mathbf{R} \mathcal{H} m_{Y}(\mathcal{F}, \mathcal{J}), \mathcal{J}\right] \\
& \cong \operatorname{Hom}_{Y}\left[\mathbf{R} f_{*}\left(\mathcal{E} \mathbf{L} \otimes \mathbf{L} f^{*} \mathbf{R} \mathcal{H} \operatorname{Hom}_{Y}(\mathcal{F}, \mathcal{J})\right), \mathcal{J}\right] \\
& \cong \operatorname{Hom}_{X}\left[\mathcal{E} \mathbf{L} \otimes \mathbf{L} f^{*} \mathbf{R} \mathcal{H} \operatorname{Hom}_{Y}(\mathcal{F}, \mathcal{J}), f^{!} \mathcal{J}\right] \\
& \cong \operatorname{Hom}_{X}\left[\mathcal{E}, \mathbf{R} \mathcal{H} \operatorname{Hom}_{X}\left(\mathbf{L} f^{*} \mathbf{R} \mathcal{H} \operatorname{Hom}_{Y}(\mathcal{F}, \mathcal{J}), f^{!} \mathcal{J}\right)\right] ;
\end{aligned}
$$

the first isomomorphism is by Lemma 3.5(ii), the second because $\mathbf{R} \mathcal{H}$ om is right adjoint to the tensor, the third by the projection formula, the fourth because $f^{!}$is right adjoint to $\mathbf{R} f_{*}$, and the fifth by the adjunction between tensor and $\mathbf{R} \mathcal{H}$ om. Put together, we deduce a natural isomorphism

$$
f^{!} \mathcal{F} \cong \mathbf{R} \mathcal{H}_{0} m_{X}\left(\mathbf{L} f^{*} \mathbf{R} \mathcal{H} \operatorname{Hom}_{Y}(\mathcal{F}, \mathcal{J}), f^{!} \mathcal{J}\right) .
$$

We have found a formula $(*)$ for computing $f^{!} \mathcal{F}$, as long as $\mathcal{F}$ belongs to $\mathbf{D}^{b}(\mathrm{Coh} / Y)$. In the old days the right hand side was used to construct the functor $f^{!}$; in our modern day and age this seems anachronistic. We know, for purely formal reasons, that the functor $f^{!}: \mathbf{D}(\mathrm{Q} \operatorname{coh} / Y) \longrightarrow \mathbf{D}(\mathrm{Qcoh} / X)$ exists; see [51, Example 4.2].

Next we restrict our attention to an even smaller class of morphisms $f: X \longrightarrow Y$; for the rest of the section assume $f$ is a morphism of noetherian, separated schemes, and suppose further that

(i) $\mathbf{R} f_{*}$ takes compacts to compacts.

(ii) The scheme $X$ satisfies Conjecture 4.16; for all we know this is no restriction.

From [46, Corollary 4.3.2] it follows that

(iii) $\mathbf{R} f_{*}$ takes $\mathbf{D}^{b}(\mathrm{Coh} / X)$ to $\mathbf{D}^{b}(\mathrm{Coh} / Y)$.

Lemma 4.4 informs us that $f^{!} \mathcal{O}_{Y}$ belongs to $\mathcal{S}(X)$, and, using (i) and (ii) above as well as Lemma 4.21 , we conclude that $f^{!} \mathcal{O}_{Y}$ is in $\mathbf{D}^{-}(\mathrm{Coh} / X)$. We therefore deduce

(iv) $f^{!}$takes $\mathbf{D}^{b}(\mathrm{Coh} / Y)$ to $\mathbf{D}^{b}(\mathrm{Coh} / X)$; see Remark 0.6 and Corollary 0.7.

(v) If $\mathcal{J}$ is a dualizing complex on $Y$, then $f^{!} \mathcal{J}$ is a dualizing complex on $X$; see Fact 0.3(ii).

If we look at (iii) and (iv), they tell us that the adjoint pair of functors $\left(\mathbf{R} f_{*}, f^{!}\right)$restrict to functors between the subcategories $\mathbf{D}^{b}(\mathrm{Coh} / X) \subset$ $\mathbf{D}(\mathrm{Qcoh} / X)$ and $\mathbf{D}^{b}(\mathrm{Coh} / Y) \subset \mathbf{D}(\mathrm{Qcoh} / Y)$; the restrictions must also be an adjoint pair. The formula $\left(^{*}\right)$ even tells us how to compute $f^{!}$in terms of dualizing complexes. 
In practice this means that, if it happens to be more convenient to compute $f^{!}$in the bounded derived categories, then go right ahead; from the restriction of $f^{!}$to $\mathbf{D}^{b}(\mathrm{Coh} / Y)$ we can compute $f^{!} \mathcal{O}_{Y}$, and the formula

$$
f^{!} \mathcal{S}=f^{!} \mathcal{O}_{Y} \mathbf{L} \otimes \mathbf{L} f^{*} \mathcal{S}
$$

tells us, at least in principle, how to work out all there is to know about the functor $f^{!}: \mathbf{D}(\mathrm{Q} \operatorname{coh} / Y) \longrightarrow \mathbf{D}(\mathrm{Q} \operatorname{coh} / X)$.

\section{Several RECEnt Results}

Let me end with a very brief glimpse at current progress in the field. We begin by observing that, up to now, our treatment has been based on studying the category $\mathbf{D}^{b}(\mathrm{Coh} / X)$ via its embedding in $\mathbf{D}(\mathrm{Qcoh} / X)$. Dualizing complexes naturally live in $\mathbf{D}^{b}(\mathrm{Coh} / X)$, and our study of them was by means of infinite coproducts, infinite products and compact objects in $\mathbf{D}(\mathrm{Qcoh} / X)$. As a result of ongoing work, we now know that there are more natural compactly generated triangulated categories to consider.

Krause [35] taught us that $\mathbf{D}^{b}(\mathrm{Coh} / X)$ can be viewed as the subcategory of compact objects in the compactly generated triangulated category $\mathbf{K}(\operatorname{Inj} / X)$. From the work of Jørgensen [29], Iyengar-Krause [27], myself $[53,54]$ and from Murfet's thesis [48], we also know a compactly generated triangulated category $\mathcal{T}=\mathbf{K}_{m}(\operatorname{Proj} / X)$ whose subcategory $\mathcal{T}^{c}$ of compact objects is naturally equivalent to $\mathbf{D}^{b}(\mathrm{Coh} / X)^{\text {op }}$. If a dualizing complex $\mathcal{J}$ exists, then tensor product with $\mathcal{J}$ gives an equivalence of categories

$$
-\otimes \mathcal{J}: \mathbf{K}_{m}(\operatorname{Proj} / X) \longrightarrow \mathbf{D}(\operatorname{Inj} / X) .
$$

This approach is very new, poorly understood, and in my opinion it has great promise. Limitations of space prevent me from discussing it any further here.

\section{Appendix A. A FACT CONCERning STRONGLY DUALIZABle OBJECTS}

In this appendix we prove the assertion of Reminder 2.7(iii); we recall the statement.

Theorem A.1. Let $\mathcal{T}$ be a compactly generated triangulated category, possessing a symmetric tensor product compatible with the triangulated structure. Assume that the unit of the tensor is compact, and that all compact objects are strongly dualizable. If $\mathcal{E} \in \mathcal{T}$ is some object, then the following are equivalent:

(i) $\mathcal{E}$ is compact.

(ii) $\mathcal{E}$ is strongly dualizable. 
(iii) Tensor product with $\mathcal{E}$ commutes with arbitrary products in $\mathcal{T}$; that is, the natural map

$$
\mathcal{E} \wedge \prod_{\lambda \in \Lambda} t_{\lambda} \longrightarrow \prod_{\lambda \in \Lambda}\left(\mathcal{E} \wedge t_{\lambda}\right)
$$

is an isomorphism for every set of objects $\left\{t_{\lambda}, \lambda \in \Lambda\right\}$.

Remark A.2. The implication (i) $\Longrightarrow$ (ii) follows immediately from the hypothesis of the theorem. The implications (ii) $\Longrightarrow$ (i) and (ii) $\Longrightarrow$ (iii) are known; for (ii) $\Longrightarrow$ (i) see [19, Theorem 2.1.3], while (ii) $\Longrightarrow$ (iii) may be found in [19, Theorem A.2.5(f)]. The fact that seems new is the implication (iii) $\Longrightarrow(\mathrm{i})$. For the convenience of the reader we give a complete, self-contained proof of the equivalence of the three conditions.

Also, because this appendix might be of interest to people who could not care less about the categories $\mathbf{D}(\mathrm{Q} \operatorname{coh} / X)$, our notation will be the standard one in the literature. The tensor product of two objects $\mathcal{E}, \mathcal{G} \in \mathcal{T}$ will be written $\mathcal{E} \wedge \mathcal{G}$, the unit of the tensor will be denoted $\mathbb{S}$, and the internal Hom-object will be $F(\mathcal{E}, \mathcal{G})$. To translate back to the case where $\mathcal{T}=\mathbf{D}(\mathrm{Qcoh} / X)$, put

$$
\mathbb{S}=\mathcal{O}_{X}, \quad \mathcal{E} \wedge \mathcal{G}=\mathcal{E}^{\mathbf{L}} \otimes \mathcal{G}, \quad F(\mathcal{E}, \mathcal{G})=\mathbf{R} \mathcal{H} \text { om }(\mathcal{E}, \mathcal{G}) .
$$

If you read again the statement of Theorem A.1 you will observe that we already used this notation in part (iii) of the theorem. With the notation established, it is time to come to the proof of Theorem A.1.

Proof. We are assuming that the compact objects are all strongly dualizable; thus

(i) $\Longrightarrow$ (ii) is trivial. Next we prove (ii) $\Longrightarrow$ (iii). Suppose therefore that $\mathcal{E}$ is strongly dualizable. In Reminder 2.7(i) we noted that there is an isomorphism $\mathcal{E} \cong\left\{\mathcal{E}^{\vee}\right\}^{\vee}$, and in Reminder 2.7(ii) we observed that $\mathcal{E}^{\vee}$ is strongly dualizable. Putting this together we have that $\mathcal{E} \wedge \mathcal{G} \cong\left\{\mathcal{E}^{\vee}\right\}^{\vee} \wedge \mathcal{G}$ must be naturally isomorphic to $F\left(\mathcal{E}^{\vee}, \mathcal{G}\right)$. We are therefore reduced to proving that the functor $F\left(\mathcal{E}^{\vee},-\right)$ respects products. But this is obvious; $F\left(\mathcal{E}^{\vee},-\right)$ is right adjoint to $-\wedge \mathcal{E}^{\vee}$.

It remains to prove (iii) $\Longrightarrow(\mathrm{i})$, which is the part that seems new. Consider the functor $\operatorname{Hom}(\mathbb{S}, \mathcal{E} \wedge-)$. The functor $\mathcal{E} \wedge-$ respects products by hypothesis and coproducts obviously. The functor $\operatorname{Hom}(\mathbb{S},-)$ respects products obviously and coproducts because we are assuming $\mathbb{S}$ compact. Therefore the composite functor $\operatorname{Hom}(\mathbb{S}, \mathcal{E} \wedge-)$ must respect both products and coproducts.

The fact that it respects products means that it must be representable; see [52, Theorem 8.6.1]. There is an object $\mathcal{G} \in \mathbf{D}(\mathrm{Qcoh} / X)$ and a natural 
isomorphism

$$
\varphi: \operatorname{Hom}(\mathcal{G},-) \longrightarrow \operatorname{Hom}(\mathbb{S}, \mathcal{E} \wedge-) .
$$

Because the functor $\operatorname{Hom}(\mathbb{S}, \mathcal{E} \wedge-)$ respects coproducts so does the isomorphic functor $\operatorname{Hom}(\mathcal{G},-)$, meaning that $\mathcal{G}$ must be compact, and therefore also strongly dualizable. Yoneda's lemma tells us that the isomorphism $\varphi$ must come from an element in

$$
\operatorname{Hom}(\mathbb{S}, \mathcal{E} \wedge \mathcal{G}) \cong \operatorname{Hom}\left(\mathbb{S}, F\left(\mathcal{G}^{\vee}, \mathcal{E}\right)\right) \cong \operatorname{Hom}\left(\mathcal{G}^{\vee}, \mathcal{E}\right) .
$$

This produces for us a morphism $\alpha: \mathcal{G}^{\vee} \longrightarrow \mathcal{E}$, which we will prove an isomorphism. What we know is that the natural map

$$
\operatorname{Hom}(\mathbb{S}, \alpha \wedge-): \operatorname{Hom}\left(\mathbb{S}, \mathcal{G}^{\vee} \wedge-\right) \longrightarrow \operatorname{Hom}(\mathbb{S}, \mathcal{E} \wedge-)
$$

is an isomorphism; it is just the map $\varphi$. In particular, for every compact object $\mathcal{K}$ we deduce that

$$
\operatorname{Hom}(\mathbb{S}, \alpha \wedge 1): \operatorname{Hom}\left(\mathbb{S}, \mathcal{G}^{\vee} \wedge \mathcal{K}^{\vee}\right) \longrightarrow \operatorname{Hom}\left(\mathbb{S}, \mathcal{E} \wedge \mathcal{K}^{\vee}\right)
$$

is an isomorphism, but this identifies with

$$
\operatorname{Hom}(\mathcal{K}, \alpha): \operatorname{Hom}\left(\mathcal{K}, \mathcal{G}^{\wedge}\right) \longrightarrow \operatorname{Hom}(\mathcal{K}, \mathcal{E}) \text {. }
$$

The compacts $\mathcal{K}$ generate, hence $\alpha$ must be an isomorphism, making $\mathcal{E}$ isomorphic to the object $\mathcal{G}^{\vee}$. We know that $\mathcal{G}$ is compact; it remains to prove that so is $\mathcal{G}^{\vee}$. But we have a natural isomorphism

$$
\operatorname{Hom}(\mathbb{S}, \mathcal{G} \wedge-) \cong \operatorname{Hom}\left(\mathcal{G}^{\vee},-\right),
$$

and the functor on the left clearly respects coproducts.

\section{REFERENCES}

1. Leovigildo Alonso Tarrío, Ana Jeremías López, and Joseph Lipman, Local homology and cohomology on schemes, Ann. Sci. École Norm. Sup. (4) 30 (1997), no. 1, 1-39.

2. Studies in duality on Noetherian formal schemes and non-Noetherian ordinary schemes, American Mathematical Society, Providence, RI, 1999.

3. Leovigildo Alonso Tarrío, Ana Jeremías López, and María José Souto Salorio, Localization in categories of complexes and unbounded resolutions, Canad. J. Math. 52 (2000), no. 2, 225-247.

4. Marcel Bökstedt and Amnon Neeman, Homotopy limits in triangulated categories, Compositio Math. 86 (1993), 209-234.

5. Alexei I. Bondal and Michel van den Bergh, Generators and representability of functors in commutative and noncommutative geometry, Mosc. Math. J. 3 (2003), no. 1, 1-36, 258.

6. Brian Conrad, Grothendieck duality and base change, Lecture Notes in Mathematics, vol. 1750, Springer-Verlag, Berlin, 2000.

7. Pierre Deligne, Cohomology à support propre en construction du foncteur $f^{!}$, Residues and Duality, Lecture Notes in Mathematics, vol. 20, Springer-Verlag, 1966, pp. 404-421. 
8. __ Cohomologie à supports propres, Théorie des topos et cohomologie étale des schémas. Tome 3 (Berlin), Lecture Notes in Mathematics, vol. 305, Springer-Verlag, 1973, (SGA 4, Exposé XVII), pp. 250-461.

9. Duiliu-Emanuel Diaconescu, Enhanced D-brane categories from string field theory, J. High Energy Phys. (2001), no. 6, Paper 16, 19.

10. Albrecht Dold and Dieter Puppe, Duality, trace, and transfer, Proceedings of the International Conference on Geometric Topology (Warsaw, 1978) (Warsaw), PWN, 1980, pp. 81-102.

11. Fouad El Zein, Complexe dualisant et applications à la classe fondamentale d'un cycle, Bull. Soc. Math. France Mém. (1978), no. 58, 93.

12. Pierre-Paul Grivel, Une démonstration du théorème de dualité de Verdier, Enseign. Math. (2) 31 (1985), no. 3-4, 227-247.

13. Alexandre Grothendieck, The cohomology theory of abstract algebraic varieties, Proc. Internat. Congress Math. (Edinburgh, 1958), Cambridge Univ. Press, New York, 1960, pp. 103-118.

14. Théorèmes de dualité pour les faisceaux algébriques cohérents, Séminaire Bourbaki, Vol. 4, Soc. Math. France, Paris, 1995, pp. Exp. no. 149, 169-193.

15. Robin Hartshorne, Residues and duality, Lecture Notes in Mathematics, vol. 20, Springer-Verlag, 1966

16. Glenn W. Hopkins, An algebraic approach to Grothendieck's residue symbol, Trans. Amer. Math. Soc. 275 (1983), no. 2, 511-537.

17. Glenn W. Hopkins and Joseph Lipman, An elementary theory of Grothendieck's residue symbol, C. R. Math. Rep. Acad. Sci. Canada 1 (1978/79), no. 3, 169-172.

18. Kentaro Hori and Johannes Walcher, D-branes from matrix factorizations, C. R. Phys. 5 (2004), no. 9-10, 1061-1070, Strings 04. Part I.

19. Mark Hovey, John H. Palmieri, and Neil P. Strickland, Axiomatic stable homotopy theory, vol. 128, Memoirs AMS, no. 610, Amer. Math. Soc., 1997.

20. Reinhold Hübl and Ernst Kunz, Integration of differential forms on schemes, J. Reine Angew. Math. 410 (1990), 53-83.

21. _ Regular differential forms and duality for projective morphisms, J. Reine Angew. Math. 410 (1990), 84-108.

22. Reinhold Hübl and Pramathanath Sastry, Regular differential forms and relative duality, Amer. J. Math. 115 (1993), no. 4, 749-787.

23. Luc Illusie, Conditions de finitude, Théorie des intersections et théorème de RiemannRoch, Springer-Verlag, Berlin, 1971, Séminaire de Géométrie Algébrique du BoisMarie 1966-1967 (SGA 6, Exposé III), pp. 222-273. Lecture Notes in Mathematics, Vol. 225.

24. _ Existence de résolutions globales, Théorie des intersections et théorème de Riemann-Roch, Springer-Verlag, Berlin, 1971, Séminaire de Géométrie Algébrique du Bois-Marie 1966-1967 (SGA 6, Exposé II), pp. 160-221. Lecture Notes in Mathematics, Vol. 225.

25. __ Généralités sur les conditions de finitude dans les catégories dérivées, Théorie des intersections et théorème de Riemann-Roch, Springer-Verlag, Berlin, 1971, Séminaire de Géométrie Algébrique du Bois-Marie 1966-1967 (SGA 6, Exposé I), pp. 78-159. Lecture Notes in Mathematics, Vol. 225.

26. _ Groupes de grothendieck des topos annelés, Théorie des intersections et théorème de Riemann-Roch, Springer-Verlag, Berlin, 1971, Séminaire de Géométrie Algébrique du Bois-Marie 1966-1967 (SGA 6, Exposé IV), pp. 274-296. Lecture Notes in Mathematics, Vol. 225. 
27. Srikanth Iyengar and Henning Krause, Acyclicity versus total acyclicity for complexes over Noetherian rings, Documenta Math. 11 (2006), 207-240.

28. Peter Jørgensen, Serre-duality for tails(A), Proc. Amer. Math. Soc. 125 (1997), no. 3, $709-716$.

29. (2005), no. 1, 223-232.

30. Anton N. Kapustin and Yi Li, D-branes in Landau-Ginzburg models and algebraic geometry, J. High Energy Phys. (2003), no. 12, 005, 44 pp. (electronic).

31. Anton N. Kapustin and Dmitri O. Orlov, Lectures on mirror symmetry, derived categories, and D-branes, Uspekhi Mat. Nauk 59 (2004), no. 5(359), 101-134.

32. Bernhard Keller, Deriving DG categories, Ann. Sci. École Norm. Sup. (4) 27 (1994), no. 1, 63-102.

33. G. Maxwell Kelly, Many-variable functorial calculus. I, Coherence in categories, Springer, Berlin, 1972, pp. 66-105. Lecture Notes in Math., Vol. 281.

34. Reinhardt Kiehl, Ein "Descente"-Lemma und Grothendiecks Projektionssatz für nichtnoethersche Schemata, Math. Ann. 198 (1972), 287-316.

35. Henning Krause, The stable derived category of a Noetherian scheme, Compos. Math. 141 (2005), no. 5, 1128-1162.

36. Ernst Kunz, Residuen von Differentialformen auf Cohen-Macaulay-Varietäten, Math. Z. 152 (1977), no. 2, 165-189.

37. 94 (1992), no. 4, 170-188.

38. Geometric applications of the residue theorem on algebraic curves, Algebra, arithmetic and geometry with applications (West Lafayette, IN, 2000), Springer, Berlin, 2004, pp. 565-589.

39. Ernst Kunz and Rolf Waldi, Regular differential forms, Contemporary Mathematics, vol. 79, American Mathematical Society, Providence, RI, 1988.

40. L. Gaunce Lewis, Jr., J. Peter May, Mark Steinberger, and James E. McClure, Equivariant stable homotopy theory, Lecture Notes in Mathematics, vol. 1213, SpringerVerlag, Berlin, 1986, With contributions by J. E. McClure.

41. Joseph Lipman, Notes on derived categories and Grothendieck duality, www.math.purdue.edu/ lipman.

42. , Dualizing sheaves, differentials and residues on algebraic varieties, Astérisque (1984), no. 117, ii+138

43. Residues and traces of differential forms via Hochschild homology, Contemporary Mathematics, vol. 61, American Mathematical Society, Providence, RI 1987.

44. L__ Lectures on local cohomology and duality, Local cohomology and its applications (Guanajuato, 1999), Lecture Notes in Pure and Appl. Math., vol. 226, Dekker, New York, 2002, pp. 39-89.

45. Joseph Lipman, Suresh Nayak, and Pramathanath Sastry, Pseudofunctorial behavior of Cousin complexes on formal schemes, Variance and duality for Cousin complexes on formal schemes, Contemp. Math., vol. 375, Amer. Math. Soc., Providence, RI, 2005, pp. 3-133.

46. Joseph Lipman and Amnon Neeman, Quasi-perfect scheme maps and boundedness of the twisted inverse image functor, Illinois J. Math. 51 (2007), 209-236.

47. Ralf Meyer and Ryszard Nest, The Baum-Connes conjecture via localisation of categories, Topology 45 (2006), no. 2, 209-259. 
48. Daniel S. Murfet, The mock homotopy category of projectives and Grothendieck duality, (PhD thesis, Aust. National U.).

49. Suresh Nayak, Pasting pseudofunctors, Variance and duality for Cousin complexes on formal schemes, Contemp. Math., vol. 375, Amer. Math. Soc., Providence, RI, 2005, pp. 195-271.

50. Amnon Neeman, The connection between the K-theory localisation theorem of Thomason, Trobaugh and Yao, and the smashing subcategories of Bousfield and Ravenel, Ann. Sci. École Normale Supérieure 25 (1992), 547-566.

51. _ The Grothendieck duality theorem via Bousfield's techniques and Brown representability, Jour. Amer. Math. Soc. 9 (1996), 205-236.

52. $ـ$ Triangulated Categories, Annals of Mathematics Studies, vol. 148, Princeton University Press, Princeton, NJ, 2001.

53. _ The homotopy category of flat modules, and Grothendieck duality, (Preprint).

54. S_ Some adjoints in homotopy categories, (Preprint).

55. Dieter Puppe, On the structure of stable homotopy theory, Colloquium on algebraic topology, Aarhus Universitet Matematisk Institut, 1962, pp. 65-71.

56. Jean-Pierre Ramis, Gabriel Ruget, and Jean-Louis Verdier, Dualité relative en géométrie analytique complexe, Invent. Math. 13 (1971), 261-283.

57. Neantro Saavedra Rivano, Catégories Tannakiennes, Springer-Verlag, Berlin, 1972, Lecture Notes in Mathematics, Vol. 265.

58. Pramathanath Sastry, Base change and Grothendieck duality for Cohen-Macaulay maps, Compos. Math. 140 (2004), no. 3, 729-777.

59. _ Duality for Cousin complexes, Variance and duality for Cousin complexes on formal schemes, Contemp. Math., vol. 375, Amer. Math. Soc., Providence, RI, 2005, pp. 137-192.

60. Jean-Pierre Serre, Un théorème de dualité, Comment. Math. Helv. 29 (1955), 9-26.

61. Nicolas Spaltenstein, Resolutions of unbounded complexes, Compositio Math. 65 (1988), no. 2, 121-154.

62. Robert W. Thomason and Thomas F. Trobaugh, Higher algebraic K-theory of schemes and of derived categories, The Grothendieck Festschrift (a collection of papers to honor Grothendieck's 60'th birthday), vol. 3, Birkhäuser, 1990, pp. 247-435.

63. Michel van den Bergh, Existence theorems for dualizing complexes over noncommutative graded and filtered rings, J. Algebra 195 (1997), no. 2, 662-679.

64. Jean-Louis Verdier, Le théorème de dualité de Poincaré, C. R. Acad. Sci. Paris 256 (1963), 2084-2086.

65. A duality theorem in the etale cohomology of schemes, Proc. Conf. Local Fields (Driebergen, 1966), Springer, Berlin, 1967, pp. 184-198.

66. Jean-Louis Verdier, Base change for twisted inverse images of coherent sheaves, vol. Collection: Algebraic Geometry, Tata Inst. Fund. Res., 1968, pp. 393-408.

67. _ Des catégories dérivées des catégories abeliennes, Asterisque, vol. 239, Société Mathématique de France, 1996 (French).

68. Amnon Yekutieli, Dualizing complexes over noncommutative graded algebras, J. Algebra 153 (1992), no. 1, 41-84.

69. An explicit construction of the Grothendieck residue complex, Astérisque (1992), no. 208, 127, With an appendix by Pramathanath Sastry.

70. Amnon Yekutieli and James J. Zhang, Dualizing complexes and perverse modules over differential algebras, Compos. Math. 141 (2005), no. 3, 620-654. 
71. _ Dualizing complexes and perverse sheaves on noncommutative ringed schemes, Selecta Math. (N.S.) 12 (2006), no. 1, 137-177.

Centre for Mathematics and its Applications, Mathematical Sciences Institute, John Dedman Building, The Australian National University, Canberra, ACT 0200, AUSTRALIA

E-mail address: Amnon.Neeman@anu.edu.au 